\title{
BLOCKING READER: DESIGN AND IMPLEMENTATION OF A PASSIVE UHF RFID BLOCKING READER
}

\author{
by \\ GAUROV NARAYANASWAMY
}

\author{
Presented to the Faculty of the Graduate School of \\ The University of Texas at Arlington in Partial Fulfillment \\ of the Requirements \\ for the Degree of
}

MASTER OF SCIENCE IN ELECTRICAL ENGINEERING

THE UNIVERSITY OF TEXAS AT ARLINGTON

December 2009 
Copyright (c) by GAUROV NARAYANASWAMY 2009

All Rights Reserved 
To my Mother Kantha and my Father K.Narayanaswamy who set an example and made me who I am. 


\section{ACKNOWLEDGEMENTS}

I joined UTA in 2007, in my first semester I took a course under Dr. Daniel Engels. I was flabbergasted with his knowledge in RFID world. I thought I had not learnt enough just by taking his class. So I joined his research team Texas RF Innovation and Technology Center in summer 2007. Daniel's ideas and his ways of research inspired and motivated me not only in gaining knowledge in RFID world but it totally changed the ways of my research. Daniel's words were like catalyst, an impulse in my brain. Daniel has been an inspiration in my life For all these reasons I would like to thank him whole heartedly for nurturing my brain to achieve my goal.

Dr. Stephen Gibbs was my graduate advisor when I joined UTA. He paved me a path advising me through my Masters. I would like to thank Steve for introducing me to Daniel's course in spring 2007. For all this I would like to thank Steve for his patience and agreeing to be my thesis chair. I would also like to take this opportunity to thank Dr. Jonathan Bredow for guiding me in my final semester of my thesis. Jon in my final semester took Daniel's place, kept constant touch with me and made sure I complete my thesis. For all this I once again thank Jon for being there when Daniel was not around. I would like to thank Dr. John Priest for supporting me and agreeing to be a part of my thesis committee.

Also I would like to thank Stanley Howard for supporting me in providing an Graduate Teaching Assistantship all through my Masters and allowing me to use his Labs for experiments. Stan has always motivated me with my work and given me a 
lot of freedom to explore. For all this I would like to thank Stan for his financial and mental support for my thesis.

Finally I would like to thank all my Family, my Father K.Narayanaswamy has always been my motivation, my Mother Kantha for her astounding affection, my sister Archana, my bro-in-law Prasad for their support through my Masters and my younger brother Abishek for his good will. Would like to take this opportunity to thank Shesh and Sachi for helping me in doing my experiments. Also would thank Amit, Pranav, Jason, Sai, Kirthi, Tanvi for making my experience in Texas Radio Frequency Innovation and Technology Center and all my friends for making my experience at UTA a joyous one.

November 19, 2009 


\title{
ABSTRACT \\ BLOCKING READER: DESIGN AND IMPLEMENTATION OF A PASSIVE UHF RFID BLOCKING READER
}

\author{
GAUROV NARAYANASWAMY, M.S.
}

The University of Texas at Arlington, 2009

Supervising Professor: Daniel W. Engels

In this thesis I present a low-cost passive UHF ISO 18000-6C Radio Frequency Identification, or RFID, reader that is feasible for mobile and privacy applications. Low cost readers are necessary to achieve the promised cost savings and enhanced revenue generation that the Internet of Things promises to deliver. Low cost readers may be installed virtually everywhere, providing real-time asset monitoring and a host of new and innovative applications yet to be conceived. While pervasively deployed readers provide tremendous benefits, the promiscuous nature of the $18000-6 \mathrm{C}$ tags means that the readers also pose a threat to personal security and privacy. Privately owned tags on a person will be read by all nearby readers without that person's consent thereby violating that person's privacy. A low cost reader worn on a person's body can be used as a Blocking Reader that prevents these unauthorized tag reads, preserving your privacy. My low- cost reader has been implemented based upon the Chipcon CC1101 chip, and I have characterized its basic read performance. using a monopole antenna, my low cost reader can be utilized as a blocking reader that prevents unauthorized reads of tags within $1 \mathrm{~m}$ of the reader. Furthermore, when 
worn on a person's body, my low-cost reader effectively acts as a Blocking Reader preventing all nearby readers from reading tags located on or in close proximity to the body. 


\section{TABLE OF CONTENTS}

ACKNOWLEDGEMENTS ............................. iv

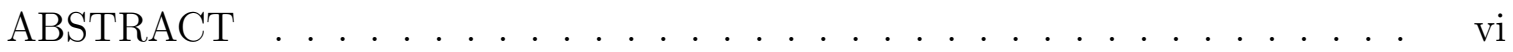

LIST OF FIGURES . . . . . . . . . . . . . . . . . . . xiii

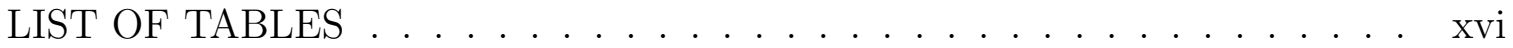

Chapter Page

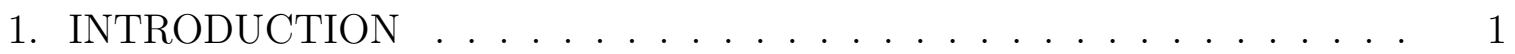

1.1 Introduction . . . . . . . . . . . . . . . . . . 1

1.2 The Threat to Privacy . . . . . . . . . . . . . . . . . 2

1.3 Blocking Reader(BR) . . . . . . . . . . . . . . . . 4

1.4 Previous Work . . . . . . . . . . . . . . . . . . 5

1.4.1 Blocker Tag ................. . . 5

1.4.2 Killer Tag approach . . . . . . . . . . . . . 6

1.4.3 Faraday Cage approach . . . . . . . . . . . . 6

1.4.4 Active Jamming approach . . . . . . . . . . . . . . 6

1.4.5 Smart RFID Tag approach . . . . . . . . . . . . . . . . 7

1.5 Implementation . . . . . . . . . . . . . . . . . . . . . . . 8

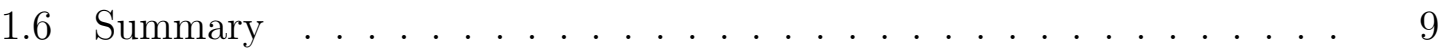

2. ELECTROMAGNETIC'S OF RFID . . . . . . . . . . . . . . . . 10

2.1 Introduction . . . . . . . . . . . . . . . . . . 10

2.2 Basic RFID System . . . . . . . . . . . . . . . . . 10

2.3 Electric Field . . . . . . . . . . . . . . . . . . . . . . 11

2.3.1 Gauss's theorem of Electric field . . . . . . . . . . . . . . . 13 
2.4 Magnetic Field . . . . . . . . . . . . . . . . . . . . . 13

2.4 .1 Ampers Law . . . . . . . . . . . . . . . . . . . . . 14

2.4.2 Gauss's theorem of magnetic field . . . . . . . . . . . 15

2.5 Maxwell Equations . . . . . . . . . . . . . . . . . . . . . 15

2.6 Antenna . . . . . . . . . . . . . . . . . . . . 15

2.6 .1 Reader Antenna . . . . . . . . . . . . . . . . . . . 16

2.6 .2 Tag Antenna . . . . . . . . . . . . . . . . . 18

2.7 Polarization . . . . . . . . . . . . . . . . . . . 20

2.8 Multipath . . . . . . . . . . . . . . . . . . . . . . . . 21

2.9 Friss Equation . . . . . . . . . . . . . . . . . . . . . . . . . . . . . . 22

3. THEORY OF BLOCKING . . . . . . . . . . . . . . . 23

3.1 Introduction . . . . . . . . . . . . . . . . 23

3.2 Inside a Passive RFID Tag . . . . . . . . . . . . . . . . . . 23

3.3 Reader Physics . . . . . . . . . . . . . . . . . . 25

4. ISO 180006-C GENERATION-2 PROTOCOL . . . . . . . . . . . 27

4.1 Introduction . . . . . . . . . . . . . . . . . . 27

4.2 Reader Protocol Layers . . . . . . . . . . . . . . . . . . . . 27

$4.2 .1 \quad$ Physical Layer . . . . . . . . . . . . . . . . . . . 28

4.2 .2 Tag-identification Layer $\ldots \ldots \ldots \ldots$

4.3 Reader Protocol Parameters . . . . . . . . . . . . . . . . . . 29

$4.3 .1 \quad$ Operational Frequencies . . . . . . . . . . . . . . . . . 29

4.3.2 Reader-to-Tag $(\mathrm{R} \Rightarrow \mathrm{T})$ communications . . . . . . . 30

4.3.3 Tag-to-Reader $(\mathrm{T} \Rightarrow \mathrm{R})$ communications $\ldots \ldots \ldots \ldots$

4.3.4 Link timings . . . . . . . . . . . . . . . . . . . 36

4.3.5 Reader Commands and Tag replies . . . . . . . . . . 38

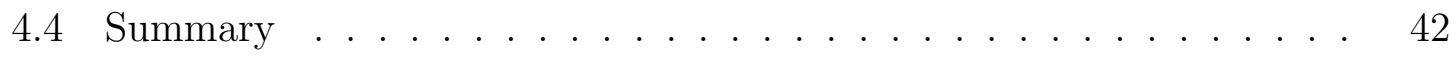


5. READER ARCHITECTURE . . . . . . . . . . . . . . . 43

5.1 Introduction . . . . . . . . . . . . . . . . . . 43

5.2 Low-Cost Reader Requirements . . . . . . . . . . . . . . . 44

5.3 Possible Low-Cost Reader Architectures . . . . . . . . . . . . . . 44

5.3 .1 Duo-PIC separate TX-RX design $\ldots \ldots \ldots \ldots \ldots$

5.3.2 Duo-PIC separate TX-RX uni-antenna design $\ldots \ldots \ldots$. . . 48

5.3.3 Uni-PIC separate TX-RX design . . . . . . . . . . . . 49

5.3.4 Uni-PIC separate TX-RX Uni-antenna design . . . . . . . . 50

5.3.5 Single processor transceiver design . . . . . . . . . . . 52

5.4 Amplifier . . . . . . . . . . . . . . . . . . 54

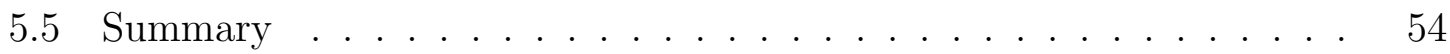

6. IMPLEMENTATION . . . . . . . . . . . . . . . . . 55

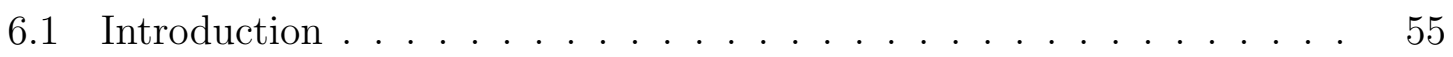

6.2 Circuit Diagram . . . . . . . . . . . . . . . . 55

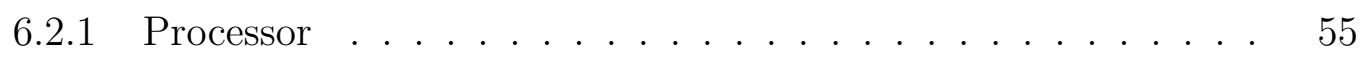

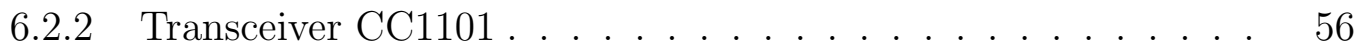

6.2 .3 Amplifier . . . . . . . . . . . . . . . . . . 63

6.3 CC1101 Register Settings _. . . . . . . . . . . . . . 64

6.3 .1 Data Rate . . . . . . . . . . . . . . . . . . . 64

6.3 .2 Frequency . . . . . . . . . . . . . . . . . . 64

6.3 .3 Bandwidth. . . . . . . . . . . . . . . . 65

6.3 .4 Modulation . . . . . . . . . . . . . . . 65

6.4 Complete Circuit Diagram . . . . . . . . . . . . . . 67

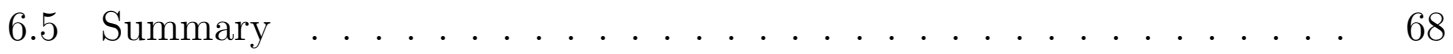

7. PERFORMANCE ......................... 69

7.1 Introduction . . . . . . . . . . . . . . . 69 
7.2 Tag Response . . . . . . . . . . . . . . . . . . . . . . . . . . . 69

$7.2 .1 \quad$ Experimental setup . . . . . . . . . . . . . . . . . 69

7.2.2 Tag Response Results . . . . . . . . . . . . . . . . . . . . 69

7.3 Maximum Tag Response Range . . . . . . . . . . . . . . . . . . 71

$7.3 .1 \quad$ Experimental setup . . . . . . . . . . . . . . . 71

7.3.2 Results for Tag Response Range . . . . . . . . . . . . . . . 72

7.4 Illustration of Blocking . . . . . . . . . . . . . . 73

7.4 .1 Experimental setup $1 \ldots \ldots \ldots \ldots \ldots$

7.4.2 Results for experimental setup $1 \ldots \ldots \ldots$. . . . . . 73

7.4 .3 Experimental setup $2 \ldots \ldots \ldots \ldots \ldots$

7.4.4 Results for Experimental setup $2 \ldots \ldots \ldots \ldots$

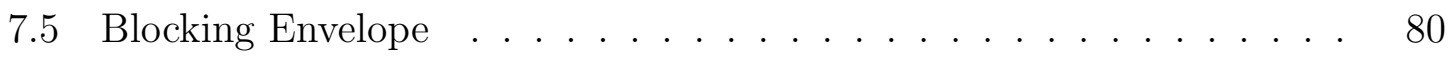

7.5.1 Blocking Envelope Around the Tag . . . . . . . . . . . 80

7.5 .2 Results . . . . . . . . . . . . . . . . . . 81

7.5.3 Blocking Envelope around the Blocking Reader . . . . . . . 82

7.5 .4 Results . . . . . . . . . . . . . . . . . . 83

7.5.5 Blocking Envelope Vertical Axis . . . . . . . . . . . . 84

7.5 .6 Results . . . . . . . . . . . . . . . . . 85

8. BLOCKING READER IN PRACTICE . . . . . . . . . . . . . 86

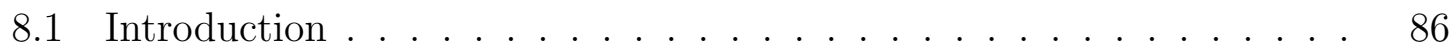

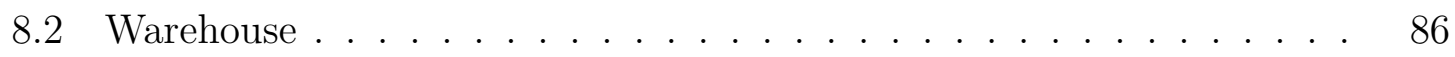

8.3 Blocking Reader Around Human Body . . . . . . . . . . . . . 87

8.3.1 Blocking Reader around human body setup . . . . . . . 90

8.3.2 Results . . . . . . . . . . . . . . . . . . . . . . . 91

9. CONCLUSIONS AND FUTURE WORK . . . . . . . . . . . . . 92

9.1 Conclusions . . . . . . . . . . . . . . . . . . . . 92 
9.2 Future Work . . . . . . . . . . . . . . . . . . 93

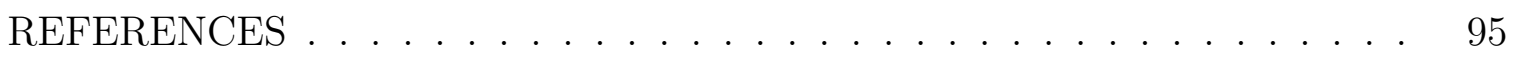

BIOGRAPHICAL STATEMENT . . . . . . . . . . . . . . . . . 101 


\section{LIST OF FIGURES}

Figure $\quad$ Page

1.1 Potential consumer privacy problems of RFID [24] . . . . . . . . . . 3

1.2 Blocking envelope around the person . . . . . . . . . . . . . . 4

2.1 A Typical RFID System . . . . . . . . . . . . . . . 11

2.2 Ampere Law: Right hand thumb rule . . . . . . . . . . . . . . . . . 14

2.3 RFID Reader antenna (picture courtesy of alien Reader) . . . . . . . 17

2.4 Patch antenna 3D gain pattern as simulated in FEKO . . . . . . . . . 17

2.5 Patch antenna gain polar plot . . . . . . . . . . . . . 17

2.6 An alien squiggle tag . . . . . . . . . . . . . . . . . 18

2.7 Gain pattern of alien squiggle tag . . . . . . . . . . . . . . . 19

2.8 FEKO 3D plot of alien squiggle tag . . . . . . . . . . . . . . 19

2.9 A typical electromagnetic wave . . . . . . . . . . . . . . 20

2.10 Multipath geometry showing EM waves reflections . . . . . . . . . . 21

3.1 Block diagram showing inside of the Tag and its filter . . . . . . . . . 24

3.2 Reader frequency hopping and slot occupancy . . . . . . . . . . . 26

4.1 Reader to Tag RF modulation envelope [3] . . . . . . . . . . . . . . . 30

4.2 Reader power-up/down RF envelope [3] . . . . . . . . . . . . . . . . 31

4.3 PIE symbols $[3] \ldots \ldots \ldots$

4.4 The Preamble and Frame-sync [3] . . . . . . . . . . . . . . . . . . . . 34

4.5 FM0 basis functions and generator state diagram [3] . . . . . . . . 36

4.6 Miller basis functions and generator state diagram [3] . . . . . . . . 36

4.7 Link timing $[3] \ldots \ldots \ldots$. . . . . . . . . . . . . . . 37 
5.1 Basic functional blocks of a RFID Reader . . . . . . . . . . . . . 43

5.2 Architecture of Duo-PIC separate TX-RX design . . . . . . . . . . 47

5.3 Architecture of Duo-PIC separate TX-RX uni-antenna design . . . . . 48

5.4 Architecture of Uni-PIC separate TX-RX design . . . . . . . . . . . 50

5.5 Architecture of Uni-PIC separate TX-RX Uni-antenna design . . . . . 51

5.6 Architecture of single processor transceiver . . . . . . . . . . . . 53

6.1 Pinout top view $[1] \ldots \ldots \ldots \ldots$

6.2 A typical application circuit for $915 \mathrm{MHz}[1] \ldots \ldots$. . . . . . . . . . . 59

6.3 Complete radio control state diagram $[1] \ldots \ldots$. . . . . . . . . . . 62

6.4 Packet format of CC1101 [1] . . . . . . . . . . . . . . 63

6.5 Application circuit diagram of RF5110 [2] . . . . . . . . . . . . 64

6.6 Complete circuit diagram of Blocking Reader . . . . . . . . . . . . . . 67

7.1 Tag Response as shown by Sniffer . . . . . . . . . . . . . . . 70

7.2 Tag Response as shown by Sniffer showing the timings . . . . . . . . . 70

7.3 Experimental setup for Tag response range . . . . . . . . . . . . 71

7.4 Tag response range . . . . . . . . . . . . . . . . 72

7.5 Experimental setup1 of Blocking Reader illustration . . . . . . . . . . 73

7.6 Graph of distance (D) vs read rate for experimental setup1 . . . . . . 74

7.7 Graph of Distance (D) vs Read Rate . . . . . . . . . . . . 76

7.8 Experimental setup2 illustrating blocking . . . . . . . . . . . . 77

7.9 Graph of read rate with BR and SR fixed . . . . . . . . . . . . 78

7.10 Comparing Friss Power for SR and BR with Read Rate . . . . . . . . 79

7.11 Setup illustrating blocking envelope around Tag . . . . . . . . . . . 80

7.12 Blocking envelope around the Tag . . . . . . . . . . . . . . 81

7.13 Setup illustrating blocking envelope around Blocking Reader . . . . . 82

7.14 Blocking envelope around the Blocking Reader . . . . . . . . . . 83 
7.15 Setup illustrating blocking envelope in vertical axis . . . . . . . . . 84

7.16 Blocking envelope in vertical axis results . . . . . . . . . . . 85

8.1 FEKO simulation of monopole antenna in front of human body . . . . 88

8.2 FEKO simulation of monopole antenna near hip of human body . . . 89

8.3 Setup for blocking reader around a human body(water bottle) . . . . 90

8.4 Maximum blocking distance measured around water bottle . . . . . . 91 


\section{LIST OF TABLES}

Table

Page

4.1 RF Envelope Parameters [3] . . . . . . . . . . . . . . . . . . . . 31

4.2 Reader power-up/down waveform parameters [3] . . . . . . . . . . 32

4.3 Preferred Tari Values $[3] \ldots \ldots$. . . . . . . . . . . . . . . . . 32

4.4 Link timing parameters $[3] \ldots \ldots$. . . . . . . . . . . . . . . . . 38

4.5 Commands $[3] \ldots \ldots \ldots$. . . . . . . . . . . . . . . . . . . 39

4.6 Select command $[3] \ldots \ldots$. . . . . . . . . . . . . . . . . . . . 39

4.7 Tag response to action parameter [3] . . . . . . . . . . . 40

4.8 Query commands $[3] \ldots \ldots$. . . . . . . . . . . . 40

4.9 QueryAdjust command $[3] \ldots \ldots$. . . . . . . . . . . . 41

4.10 QueryRep command [3] . . . . . . . . . . . . . . 41

4.11 ACK command [3] . . . . . . . . . . . . . . . . 42

4.12 NAK command $[3] \ldots \ldots \ldots$. . . . . . . . . . . . . . . . 42

5.1 Duo-PIC separate TX-RX design: parts and cost . . . . . . . . . 47

5.2 Duo-PIC separate TX-RX uni-antenna design: parts and cost . . . . . 49

5.3 Uni-PIC separate TX-RX design: parts and cost . . . . . . . . . . 50

5.4 Uni-PIC separate TX-RX Uni-antenna design: parts and cost . . . . . 51

5.5 List of Parts and Cost for Single processor transceiver design . . . . . 53

5.6 Cost comparison of different Reader designs . . . . . . . . . . . . . . 54

6.1 Bill of material for the application circuit [1] . . . . . . . . . . 60

6.2 Final register settings . . . . . . . . . . . . . 66 


\section{CHAPTER 1}

\section{INTRODUCTION}

\subsection{Introduction}

An RFID (Radio Frequency IDentification) Reader is a device whose primary purpose is to read the identifier stored on all RFID tags located near it. Reader communicates this Tag identifier to the backend information system. RFID is widely deployed in the supply chain industry. It is becoming more ubiquitous by the day. This is good for achieving the applications and such from the Internet of Things but it poses significant security and privacy concerns for individuals since the most widely deployed tags are promiscuous. This means that a person may be easily tracked without their knowledge or their consent. So how secure is the RFID wireless communication? Will the Tags communicate only to authorized Readers. Security and Privacy is an important issue in RFID systems. Low cost RFID readers will enable widely anticipated benefits, and they will also be the source of security protecting our privacy as we wonder through this ubiquitously connected world of our future. Different companies have come up with there own methods of providing security and privacy. It is astonishing how a modest device like an RFID tag essentially just a wireless license plate, can give rise to the complex melange of security and privacy problems [24]. There are some research done in providing privacy. Juels offers a survey of these problems in his paper[24]. Blocker Tag is one possible solution mentioned in his paper [6]. Also S.A. Weis proposes several security mechanisms to make the Tags smarter [44]. 
In this thesis I present the Blocking Reader. The Blocking Reader is designed to be a low-cost ISO $18000-6 \mathrm{C}$ compliant reader whose primary purpose is to legally prevent other readers from reading tags in its vicinity. The Blocking Reader communicates with the Tags and captures it. This prevents other Readers in the vicinity to communicate with this Tag. Blocking Reader is built on a Low-Cost Reader archi-

tecture. Also performance of the Blocking Reader is tested. Application of Blocking Reader on person's body (FEKO simulated Human body model) shows how it can preserve consumer's privacy.

\subsection{The Threat to Privacy}

The promiscuity of RF tags is not unique; magnetic stripe cards, for example, are promiscuous, but we assume that the owner of the card takes the physical responsibility of preventing unauthorized users from physically accessing the card [45]. It is possible to access the information of the Tag by any unauthorized reader. Even if tag contents are protected, individuals may be tracked through predictable tag responses; essentially a traffic analysis attack violating location privacy. Spoofing of tags may aid thieves or spies. Saboteurs could threaten the security of systems dependent on RFID technology through denial of service [49].

Technology is advancing at a faster pace. Need of tagging all the products for having good supply chain management is increasing. RFID has promised to provide that at a very low cost. But what are the risks of this. The Picture 1.1 shows Mr. Jones privacy is breached by knowing all his personal information. Having all this information a advertising company will make his life miserable.

In case of military it is very important to preserve the privacy of all its operations. The enhanced supply-chain visibility that makes RFID so attractive to industry 


\section{The consumer privacy problem}

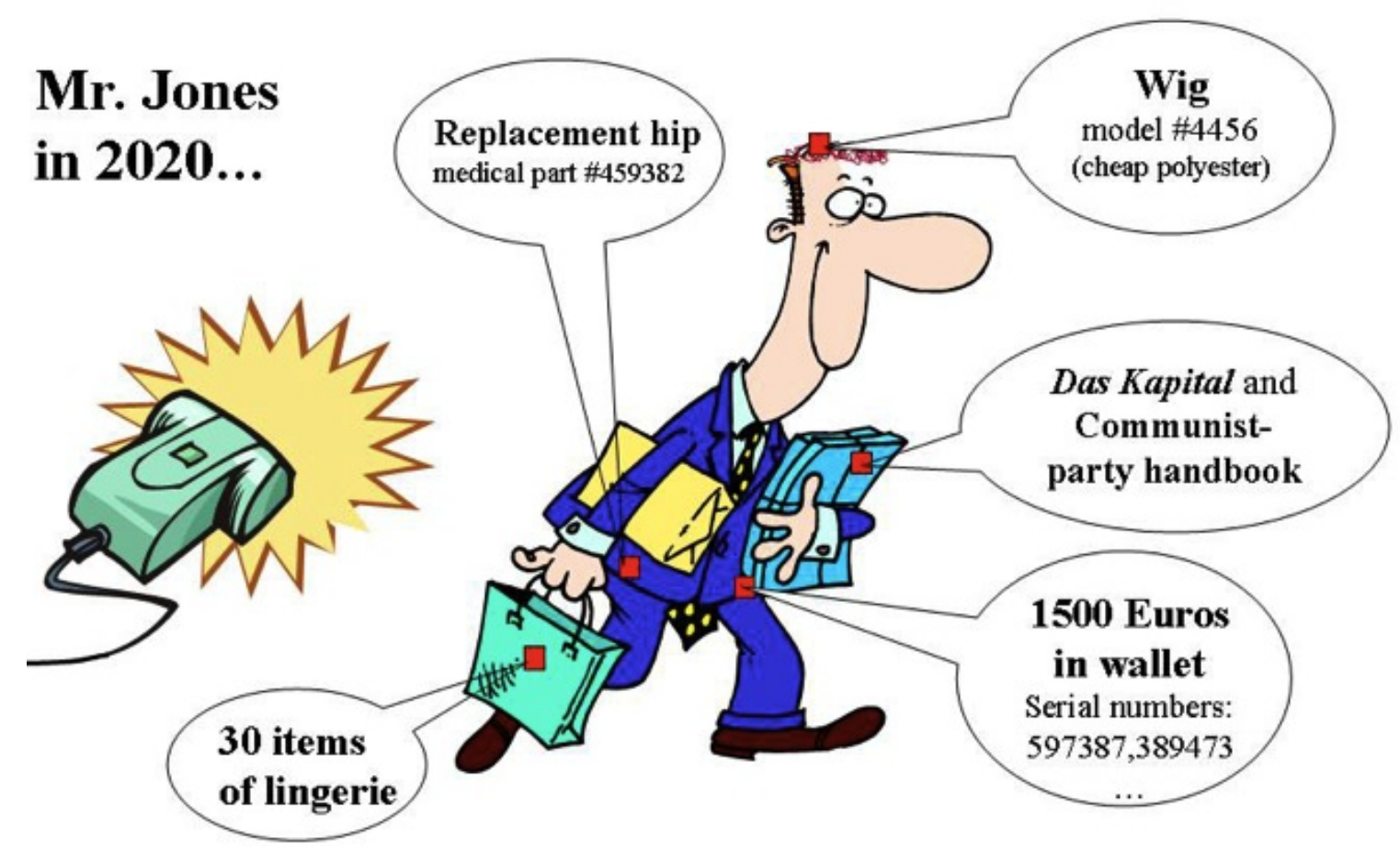

Figure 1.1. Potential consumer privacy problems of RFID [24] .

can also, in another guise, betray competitive intelligence[24]. It is a national risk for the military if the enemy forces are monitoring them.

The RFID technology has gained popularity in the supply chain industry making the products visible at real time. But there is a treat to the company if other companies can also get this information. We have already discussed how easy it is to get this information. So it is very important to ensure the privacy by making the RFID Systems more secure. In this Thesis we will discuss how we can make this happen with other devices outside the RFID system. 


\subsection{Blocking Reader(BR)}

In this thesis I present the Blocking Reader which blocks the communication between the Reader and Tags. A Blocking Reader is a Reader that prevents other unauthorized Reader from communicating with your Tags and provides a method of security to the system. The Blocking Reader is an external device that can be used in the vicinity of the RFID system and provide with the security. Hence doesn't require any reconfiguration of the RFID system. The blocking is achieved for a very good range of about $1 \mathrm{~m}$ with a monopole antenna transmitting $19-20 \mathrm{dBm}$ of power. Any tags inside this range is blocked from other Readers trying to access the information of this Tag.

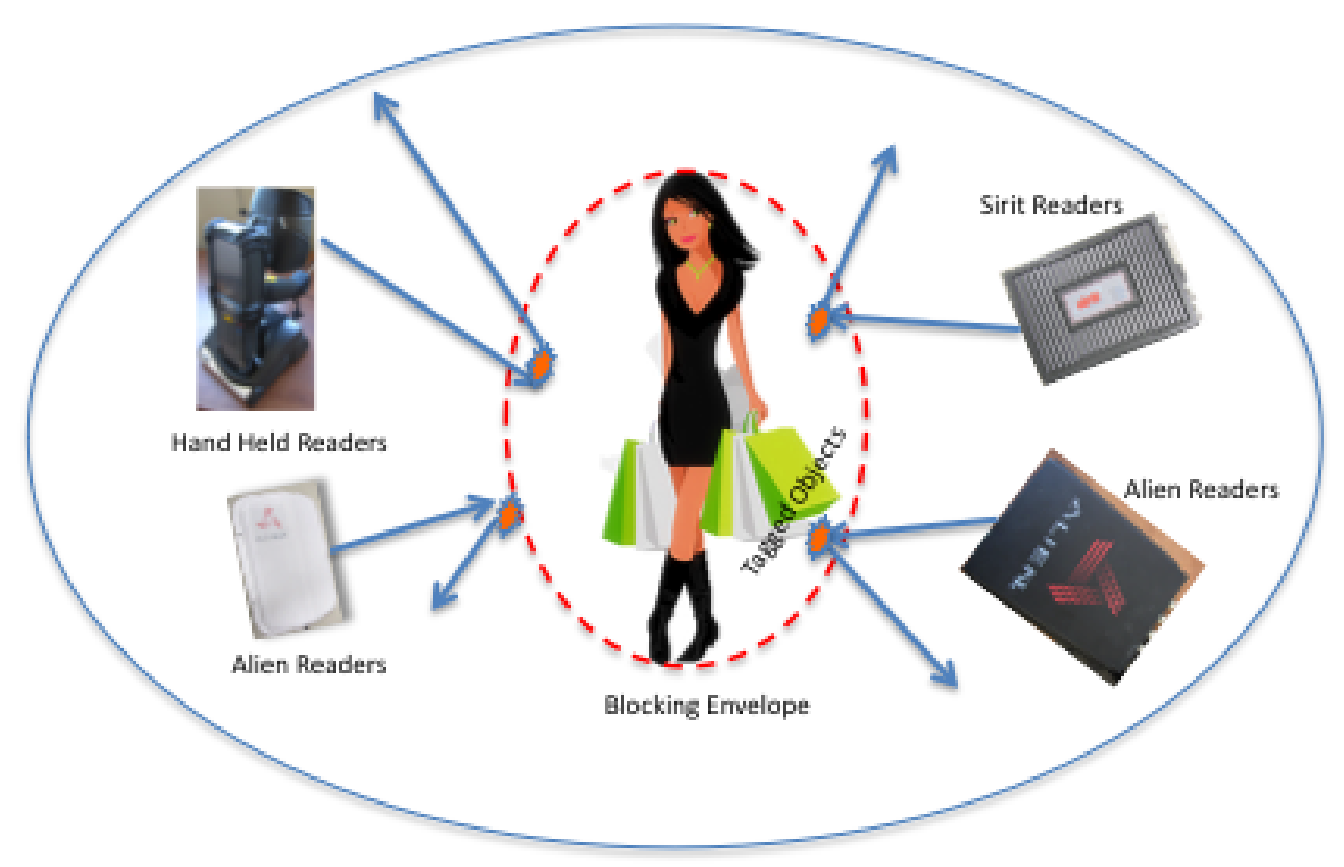

Figure 1.2. Blocking envelope around the person. 
Blocking Reader uses inventory round of the protocol to its advantage. The Blocking Reader transmits a serious of Query commands hence capturing the Tags. The Tags reply to the query with an RN16 value but instead of acknowledging the Tag, the BR transmit another Query command. Now the Tag assumes that the Reader has not received the RN16. So it sends the RN16 again. This is done continuously capturing the Tag. Blocking Reader uses a low-cost Reader architecture design. In

this thesis I present a different low-cost Reader architectures and implement BR in the simplest low-cost Reader design.

\subsection{Previous Work}

Many have come up with different ways to provide privacy and security to the RFID systems. Jules came up with a concept of Blocker Tag [24]. S.A. Weis proposes several security mechanisms to make the Tags smarter [44].

\subsubsection{Blocker Tag}

Ari Juels proposes a simple blocker-tag scheme for privacy protection [24]. Here blocker tags selectively exploit the tree-walking singulation protocol. Indeed his approach was to participate in the Tag communication in a non-compliant way causing active jamming. Here the the approach is behaving like a Tag and sending a full spectrum of serial numbers. Hence this process will obscure the serial numbers of other Tags. This makes Readers hard to singulate Tags in the field. The blocker tag when carried by a person induces a physical region of privacy protection around the person. 


\subsubsection{Killer Tag approach}

This is a decent approach to consumer privacy, the Tags are killed with the "kill" command whenever necessary as proposed by AutoID Center[45, 3]. In the RFID world all manufactured products are tagged with a unique RFID Tag to keep track of products and its identity. When the products are sold to the consumer, it can be simply be killed with a simple "kill" command. Indeed this approach is inadequate, the killed Tags can't be used. In many RFID applications require Tags to still be active, once killed tags cannot be activated. This approach is already used in practice. There is no cost as such to kill a Tag, but this is a compulsive approach similar to tampering the Tag.

\subsubsection{Faraday Cage approach}

Metal is known to reflect any RF radiations of certain frequencies. Indeed this can be used to advantage by caging the tags in a container made of metal or foil. We already know that some petty thieves use foil-lined bags for shoplifting. RFID credit cards are prone to digital pickpocketing, foil lined wallets are sold in the market at a starting cost of $\$ 19.85$ (via SkyMall.com). Also Faraday Cage approach is a physical approach.

\subsubsection{Active Jamming approach}

Radio jamming is another physical means to block Tags by actively broadcasting high power signals to decrease the signal to noise ratio. Tag jamming creates a noise shield around the Tag. In this approach an illegal broadcast of high power RF signals where done to create disturbance in communication. Hence having a Jamming effect. Unlike blocker tag which is designed to be FCC compliant. Active jamming is already 
in practice in jails where they want to jam any cell phone signals. Jamming was also used in world war II to prevent enemies from communicating.

\subsubsection{Smart RFID Tag approach}

RFID Tags can become smarter by implementing additional features on top or in the protocol making it secure. Many concepts are being proposed by S.A. Weis in his paper and thesis [44, 48].

\subsubsection{Hash-Lock approach}

In this approach $[44,48]$ the Tag are locked and refuses to give its ID unless unlocked. The unlock code being a meta-ID is only known to a authorized Reader. Hence the privacy of the Tags are preserved even after Tagged products sold to other customers. The meta-ID can be shared between Readers authorizing them to read. But how secure is this approach, snooping can retrieve the Tag ID when communicating with a authorized Reader. Also Meta-ID can be cracked. It also creates inconvenience for the consumer to handle this.

\subsubsection{Re-encryption approach}

Re-encryption approach proposed by Juels and Pappu [25]. It was mainly focused on financial cryptography. The RFID tagged bank notes are prone to privacy and security issues. The serial numbers of the Tags are encrypted with lawenforcement public key. Periodic re-encryption is done to avoid guessing of different appearances. The main drawback of this is the cost of implementation, difficult to re-encrypt every time. 


\subsubsection{Silent Tree-Walking approach}

Silent Tree-Walking approach was proposed by S.A.Weis [44]. This concept was proposed mainly to avoid the treat posed by passive eavesdroppers. By encrypting the Reader communication it is possible to avoid passive eavesdroppers to infer the Tag ID. But this approach is not suitable if another active reader is trying to communicate with the Tag.

So none of these approaches suitably preserve the privacy for personal tags. These approaches are suitable only for specific applications. But the concept of blocker Tag is exploited and instead of behaving like a Tag, the Blocking Reader behaves like a Reader. Blocking Reader uses compliant ways to achieve blocking, again inducing a physical region of privacy.

\subsection{Implementation}

The Blocking Reader is implemented with a Ultra low cost Reader architecture. It uses a CC1101 radio for RF communication. As the name says Blocking Reader is a Reader which blocks. It emulates a Reader in more efficient way hence capturing Tags causing the blocking effects. There is a potential scope for any Reader to just use this concept to turn into a Blocking reader if necessary. The Blocking Reader is designed to be FCC compliant for RFID systems, not transmitting more that $1 \mathrm{~W}$ power. Blocking Reader uses that same ISO 180006-C GEN2 protocol which Readers use. The Blocking Reader is implemented with a simple Reader architecture and is tested for its working. The performance of the Blocking Reader is tested for different experimental setups. A real time application is done in presence of a Human Body and the results are verified with the simulations done in FEKO. 


\subsection{Summary}

It is surprising that a modest device like a RFID Tag is lacking personal security. And being naked to any justified interrogators to attain secure data of the Tag. The thesis ensures privacy of the RFID Tag with a low cost ISO 18000-6C compliant Blocking Reader. BR can be used to block the communication between your Tag and any unauthorized Reader in the vicinity. A low-cost Reader design architecture is used in the Blocking Reader hence having a low cost of implementation. The BR uses inventory round of the protocol for blocking. The Tag replies to the communication of the BR. Hence the Blocking Reader is actually blocking and not jamming. Laboratory experiments prove that the BR can deliver a maximum blocking range of about $1 \mathrm{~m}$. $1 \mathrm{~m}$ of envelope around the BR is like a shied inside which no Tags can be read. BR provide personal privacy for a person wearing the decide. Laboratory experiment shows a maximum blocking envelope of about less then $1 \mathrm{~m}$ when used a 5 gallon filled water bottle. The concept of BR can be used in other RFID systems with inventory round. The BR provides scope for implementing it on the Tag. Finally Blocking Reader can provide a solution to personal privacy issues of the Tag. 


\section{CHAPTER 2}

\section{ELECTROMAGNETIC'S OF RFID}

\section{$2.1 \quad$ Introduction}

It is important to know the Electromagnetic's of Radio Frequency Identification(RFID), the physics concerning the communication between reader and tag. Knowledge of the fields and different laws governing the entire RFID System communication will help us to give more insight to analysis. The thesis consist of analysis of RF Radiation patterns of the Blocking reader. The RF front end of the blocking reader design consists of proper selection of the type of modulation, frequency and power. This chapter gives a small introduction to all the theories involved in the communication like Electric field, Magnetic field theory, equations governing them, modulation, antenna theory, multi-path and polarization.

\subsection{Basic RFID System}

A typical RFID system is as shown in the Figure 2.1. It consists of a Reader which readers the Tag information and transmits to the back-end information system for further processing. Unlike barcodes the Tags communicate wirelessly transmitting the identifier to the Reader. There are different types of RFID systems.

RFID systems can be classified based on frequency and based on communications. In this thesis we will only talk about passive UHF RFID system. UHF systems are far field operated systems where data is communicated through electromagnetic waves. UHF band allowable in US for RFID communication is 902-928 MHz. Passive RFID systems consists of Passive UHF Tags. Passive Tags are battery-less that de- 


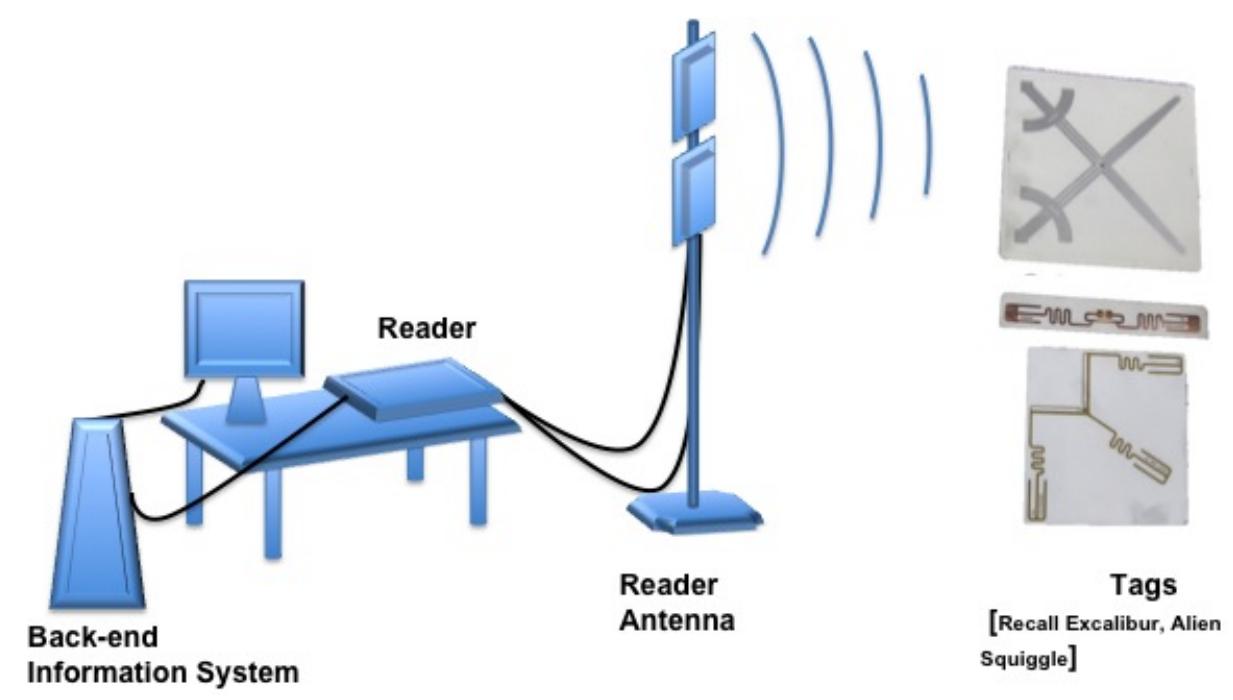

Figure 2.1. A Typical RFID System.

rives power from the Reader for communication. Some passive Tags are as shown in the Figure 2.1. ISO 180006-C GEN 2 protocol used in the passive systems is explained in chapter 4. Now let us see some physics of RFID.

\section{$2.3 \quad$ Electric Field}

The ancient Greeks observed that when the fossil resin, amber was rubbed, small light-weight objects were attracted [50][40]. Not until eighteen century it was quantified that it was due to electric charge. The electric charge is either positive or negative. Positively charged due to the lack of electrons and negatively charged due to the presence/accumulation of electronics. This charge can be placed due to electrostatic induction.

Coulomb in 1785 using his very sensitive torsion balance, on the face between like charges, followed by further investigations in 1787 to cover the attractive case, which gave direct quantitative verification of the inverse square law, it is now uni- 
versally known as Coulomb's Law [51]. Hence he saw a force field between these two bodies which can be represented from the from this equation below.

$$
F=\frac{1}{4 \pi \varepsilon_{o}} \frac{q_{1} q_{2}}{r^{2}}
$$

Here $\mathrm{F}$ is electrostatic force, $\mathrm{r}$ is the distance between the charged bodies, $\mathrm{q} 1$, q2 are the charges on the respective bodies and $\varepsilon_{o}$ is the permittivity of free space.

The electric field can be defined as force a charged body experiences per unit charge. The unit of electric field is Newton per coulomb $\left({ }^{N} / C\right)$ which is equivalent to volts per meter $(V / m)$. If $\mathrm{E}$ is the electric field, $\mathrm{F}$ is the coulombs force experienced by the charged body with charge Q then

$$
E=\frac{F}{Q}
$$

Electric field is dependent on the dielectric constant of the medium. So if $E_{0}$ is the electric field in the free space and E is the Electric field in the medium with the relative permittivity of $\varepsilon$.

$$
E=\varepsilon E_{0}
$$

if, $\varepsilon_{o}=8.854 \times 10^{-12}$ and $\varepsilon_{r}$ is the relative permittivity of the medium then,

$$
\varepsilon=\varepsilon_{0} \varepsilon_{r}
$$

The energy (U) stored by an electric field is given by

$$
U=\frac{1}{2} \varepsilon E^{2}
$$




\subsubsection{Gauss's theorem of Electric field}

Gauss's theorem relates the flux trough a closed surface S to the charge enclosed by that surface [51].

$$
\phi_{e}=\oint_{s} E \cdot d A
$$

\subsection{Magnetic Field}

Studies on electromagnetism began after Faraday's discovery of electromagnetic induction in the year 1831, a phenomenon that was later theorized by Maxwell in 1873 with the concept of fields [20]. The utilization of magnetic materials like iron, hematite, magnetite etc have been done for centuries. We all know about the navigation compass. Gilbert considered earth as a giant magnet to explain the compass.

Magnetic Field is denoted by two vectors magnetic field strength $\vec{B}$ unit $\left({ }^{A} / \mathrm{m}\right)$ and magnetic flux density $\vec{H}$ unit $\left({ }^{W} b / m^{2}\right)$. The flux density and magnetic field strength are related by permeability of the material $(\mu)$. The relation is shown in the following equation

$$
\vec{B}=\mu \vec{H}
$$

and

$$
\mu=\mu_{0} \mu_{r}
$$

Where, $\mu_{0}=4 \pi \times 10^{-7} \mathrm{H} / \mathrm{m}$. 


\subsubsection{Ampers Law}

When Oersted observed a deflection in a small compass needle in the vicinity of a wire carrying current, he taught this might be due to a magnetic field created by the wire. The Ampere law states that the magnetic field intensity, as measured by a small compass at a distance $r$ from a straight current $\vec{I}$, is described as

$$
|\vec{H}| \alpha \frac{\vec{I}}{r}
$$

for which the directions of $\vec{I}$ and vector $\vec{H}$ are related by the right hand screw rule, as shown in Figure $2.2[20]$.

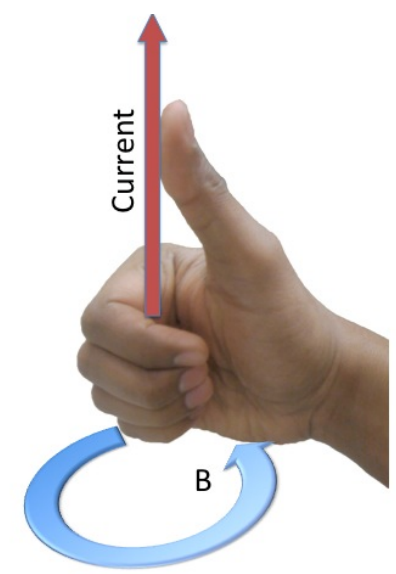

Figure 2.2. Ampere Law: Right hand thumb rule.

The Biot-Savart Law stating the relationship between the electric current flowing through the conductor and magnetic flux is as follows.

$$
H=\frac{I}{4 \pi} \int \frac{d l \times R}{R^{3}}
$$




\subsubsection{Gauss's theorem of magnetic field}

The magnetic equivalent of Gauss's theorem for the electric field for a closed surface $S$ is given by,

$$
\phi_{m}=\oint_{s} B \cdot d S=0
$$

\subsection{Maxwell Equations}

Maxwell formulated the laws of electromagnetism by hydrodynamic analogy, basing them on faraday's concept of fields [20]. These equations constitute fundamental laws of physics so every macroscopic fields obeys Maxwell's equations in their entirety. Dynamic fields are the most complex compared to Static. However, certain features of dynamic field can be analyzed as though the field were static, then the field is called as quasi-static.

$$
\begin{gathered}
\nabla \times E=-\frac{\partial B}{\partial t} \\
\nabla \times H=J+\frac{\partial D}{\partial t} \\
\nabla \cdot D=\rho \\
\nabla \cdot B=0
\end{gathered}
$$

\subsection{Antenna}

Antennas serve as transducers between electromagnetic waves traveling in free space and guided electromagnetic signal in circuits [22]. Basically antennas acts as a interface that converts wired systems into wireless systems. So it is important to have 
good knowledge of this device which has a very important role in the performance of a system. An RFID system is a wireless system, with power less that $1 \mathrm{~W}$ for passive systems, it is very important for any design engineer to have knowledge of basic antenna operational parameters including input impedance, efficiency, radiation pattern, directivity, gain and noise temperature. In this section we will very briefly discuss about different types of antennas used in RFID systems and its gain. The reader antennas must be very directional and sensitive. And tag antennas must be wide-band, in some cases low cost compact design. In RFID system there are two sets of antennae used, one is with the reader and other in the tag.

\subsubsection{Reader Antenna}

Reader antennas should be directional and sensitive as mentioned earlier. Most of the time multiple antennas are connected to the Reader. The reader antenna is mostly a smart antenna where the gain, sensitivity, protocol parameters can be changed. There are readers like Voyantic reader which can be used to do tag analysis, environment analysis by changing these parameters. An EPC-compliant antenna costs up to $\$ 500$ [11]. Some of the reader antennas are mentioned below.

- Coil Antennas

- Patch Antennas

- Etched Antennas

- Monopole Antennas

- Lens Antennas

- Fractal Antennas

Figure 2.3 shows a Alien linear antenna with 5.90dbi gain and operates at 902928MHz. A Patch antenna is designed in FEKO and simulated for $915 \mathrm{Mhz}$ and the radiation patterns are as shown below. 


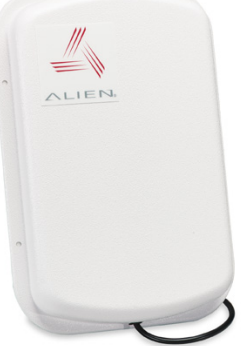

Figure 2.3. RFID Reader antenna (picture courtesy of alien Reader).

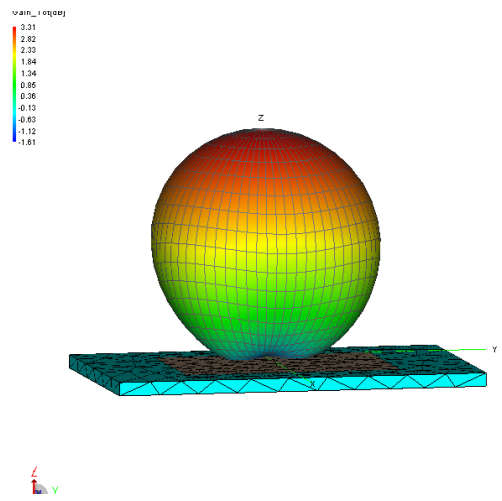

Figure 2.4. Patch antenna 3D gain pattern as simulated in FEKO.

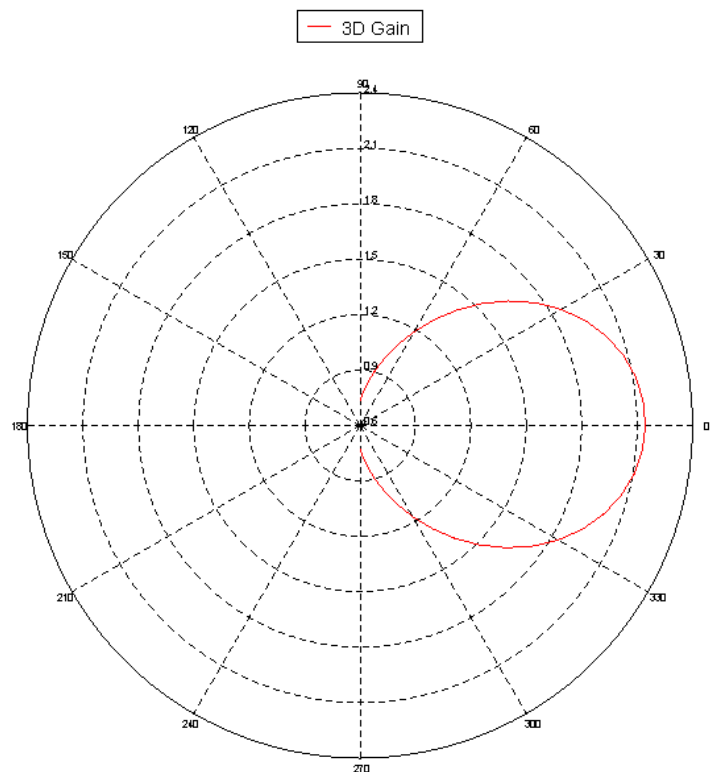

Figure 2.5. Patch antenna gain polar plot. 


\subsubsection{Tag Antenna}

Tag antennas as we mentioned earlier should be wide band and is constrained to cost. Due to this a lot of research is done in this field and there are thousands of antenna designs. The tag antennae are usually connected to the chip directly. Most commonly used tag antennas are

- Coil Antennas

- Etched Antennas

- Monopole Antennas

- Inlay Antennas

A alien squiggle inlay ALN-9640 tag is shown in Figure 2.6. The radiation pattern simulated by FEKO is shown in Figure 2.7

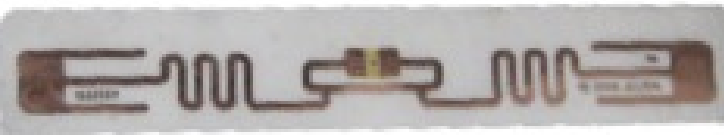

Figure 2.6. An alien squiggle tag. 


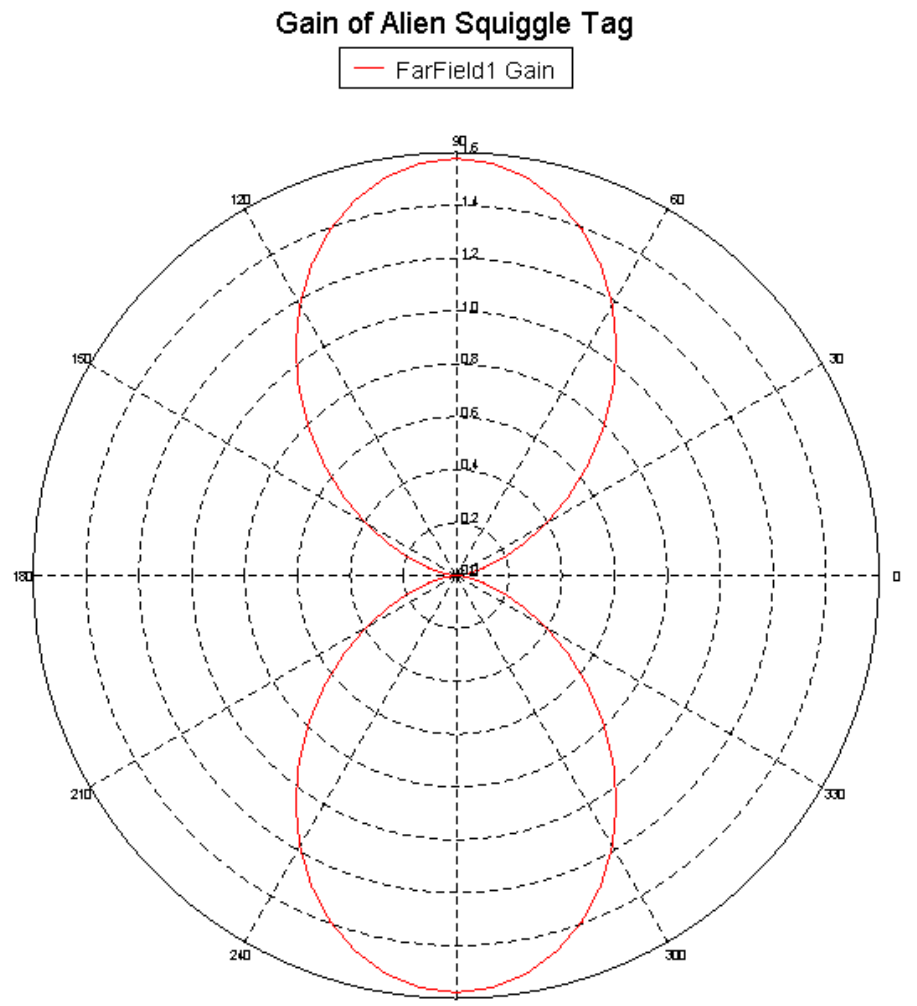

Figure 2.7. Gain pattern of alien squiggle tag.

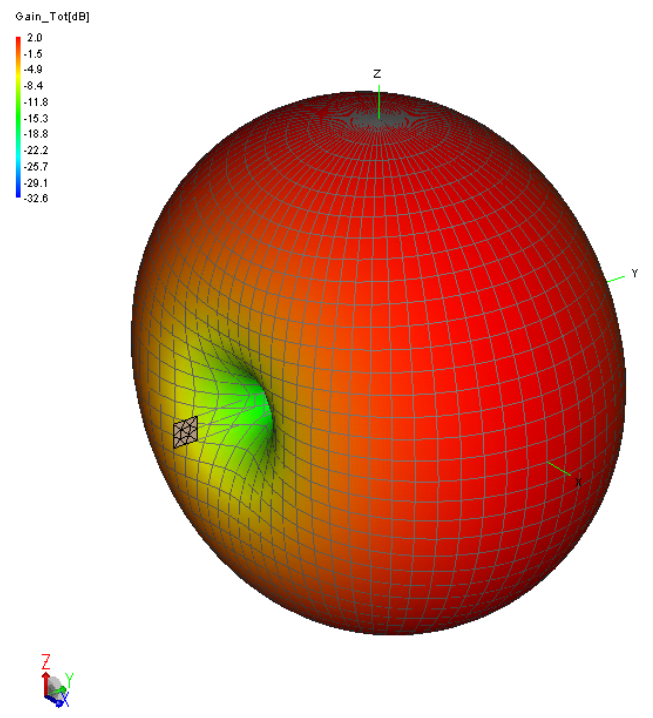

Figure 2.8. FEKO 3D plot of alien squiggle tag. 


\subsection{Polarization}

The electromagnetic field consists of electric and magnetic field vectors which are orthogonal to each other as show in the Figure 2.9. The property of waves which orients itself during oscillations is called polarization. There are different types of polarizations they are

- Linear

- Circular

- Elliptical

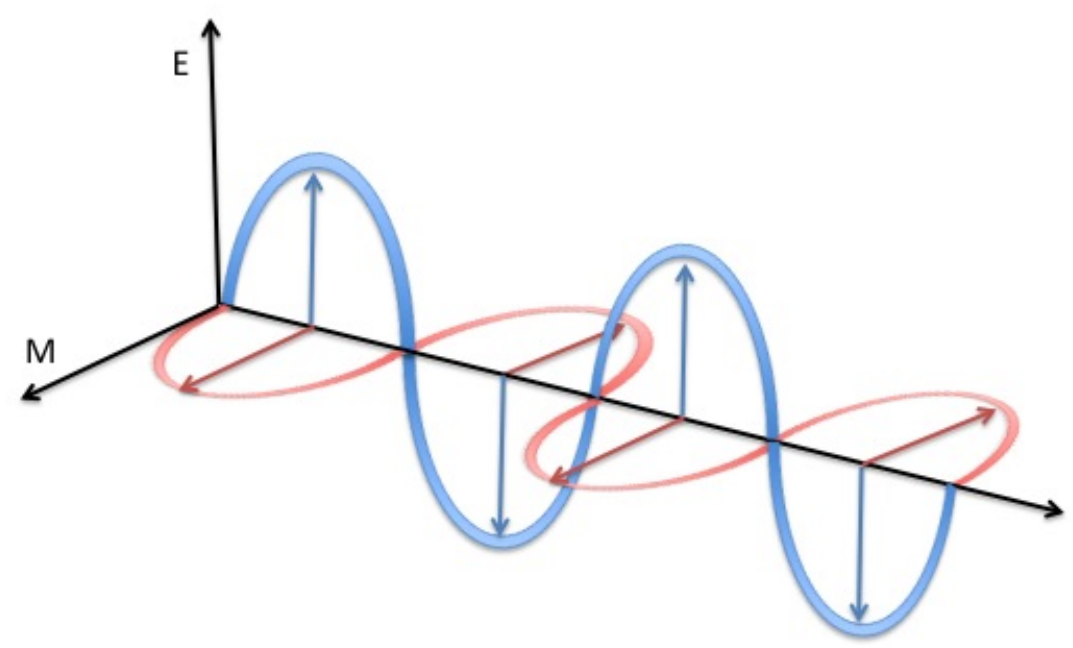

Figure 2.9. A typical electromagnetic wave.

Based on the application of RFID systems the type of the polarization is considered. It is very important to have knowledge of polarization in RFID because, the readability of the tag depends on the orientation of the tag. 


\subsection{Multipath}

The term multipath denotes the possibility of EM wave propagation along various paths from the transmitter to the receiver [9]. Due to multipath the radiation pattern of the transmitter changes. In RFID if the radiation pattern of the reader antenna changes the readability of the reader changes. This is the sole reason for the difference in the radiation pattern of the reader in different environment. The reflection, absorption of radiations by the objects around the reader constitute the environment characterization. Even presence of human changes the radiation pattern of the antenna [14]. The effect of multipath increases with the distance between the transmitter and receiver. In this thesis we have done simulation experiments of human body showing how the radiation pattern changes. One of the reason is the reflection of $\mathrm{RF}$ radiations around the human body.

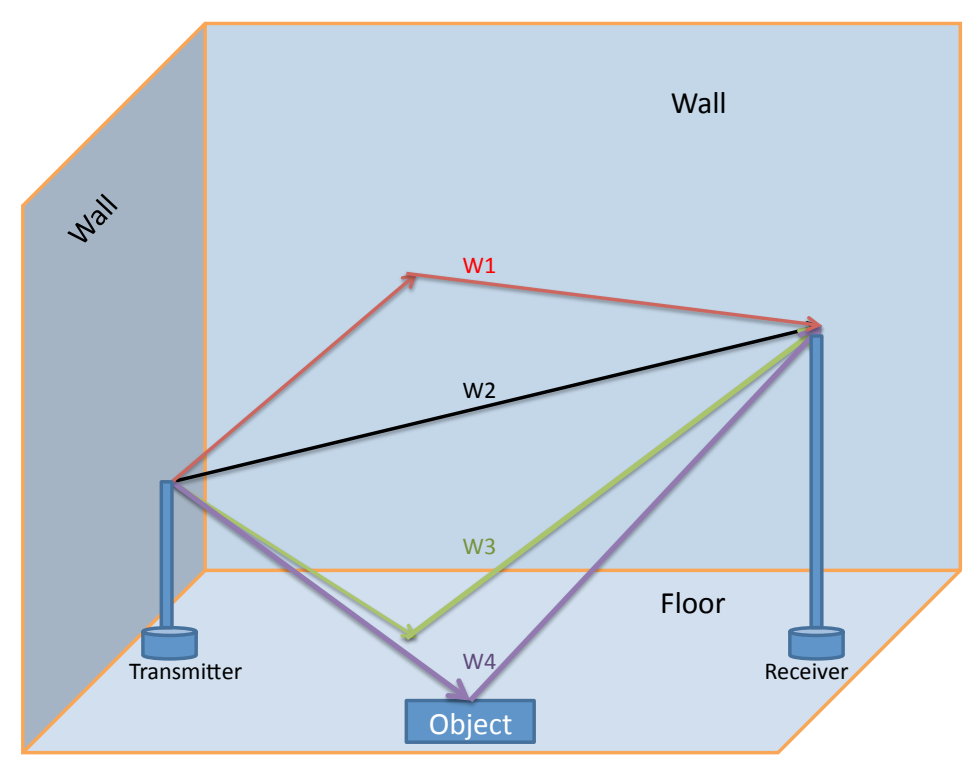

Figure 2.10. Multipath geometry showing EM waves reflections. 


\subsection{Friss Equation}

Friis equation represents the power relation between transmitter antenna power and receiver antennas power as shown in the Equation 2.16. This equation is applicable only in ideal conditions. So it is assumed that the antennas are in unobstructed free space and there is no multipath. The received power $P_{r}$ at the receiver antenna placed at a distance $\mathrm{R}$ can hence be calculated if we know $P_{t}$ the power transmitted and gains of transmitter $\left(G_{t}\right)$ and $\operatorname{receiver}\left(G_{r}\right)$ antennas.

$$
P_{r}=P_{t} G_{t} G_{r}\left(\frac{\lambda}{4 \pi R}\right)^{2}
$$




\section{CHAPTER 3}

\section{THEORY OF BLOCKING}

\subsection{Introduction}

In this chapter we will discuss about the theory behind Blocking. Previous work from Juels, S.A. Wies and others came up with different approaches to provide privacy and security to the RFID systems as discussed in section 1.4. Some concepts and approaches like killer Tag, smart Tag are application specific, Tag jamming is illegal and faraday's cage has practical limitations. So in this thesis we have a new approach to blocking called Blocker Reader where we block the Readers from reading the Tag using Tag capture approach. This approach is similar to blocker tag but here blocking reader acts like a Reader. An external device called Blocking Reader captures your Tags and not allow other Readers to Read the Tag. In this chapter we discuss the concept of Tag capture and see how the Blocking Reader use this to block the Readers.

\subsection{Inside a Passive RFID Tag}

A passive UHF RFID Tag has no battery to power. It uses the carrier wave power from the Reader to energize the circuitry and transmit the encoded ID. So the

Tags reply at the same frequency as the Reader. Tags antennas are hence designed to be wide-band in $902-928 \mathrm{MHz}$. Typically tuned to have maximum gain at center frequency $915 \mathrm{MHz}$.

Figure 3.1 shows some basic blocks that are present inside a passive Tag. Passive Tags now come as a single chip IC connected to an antenna. Inside the chip 


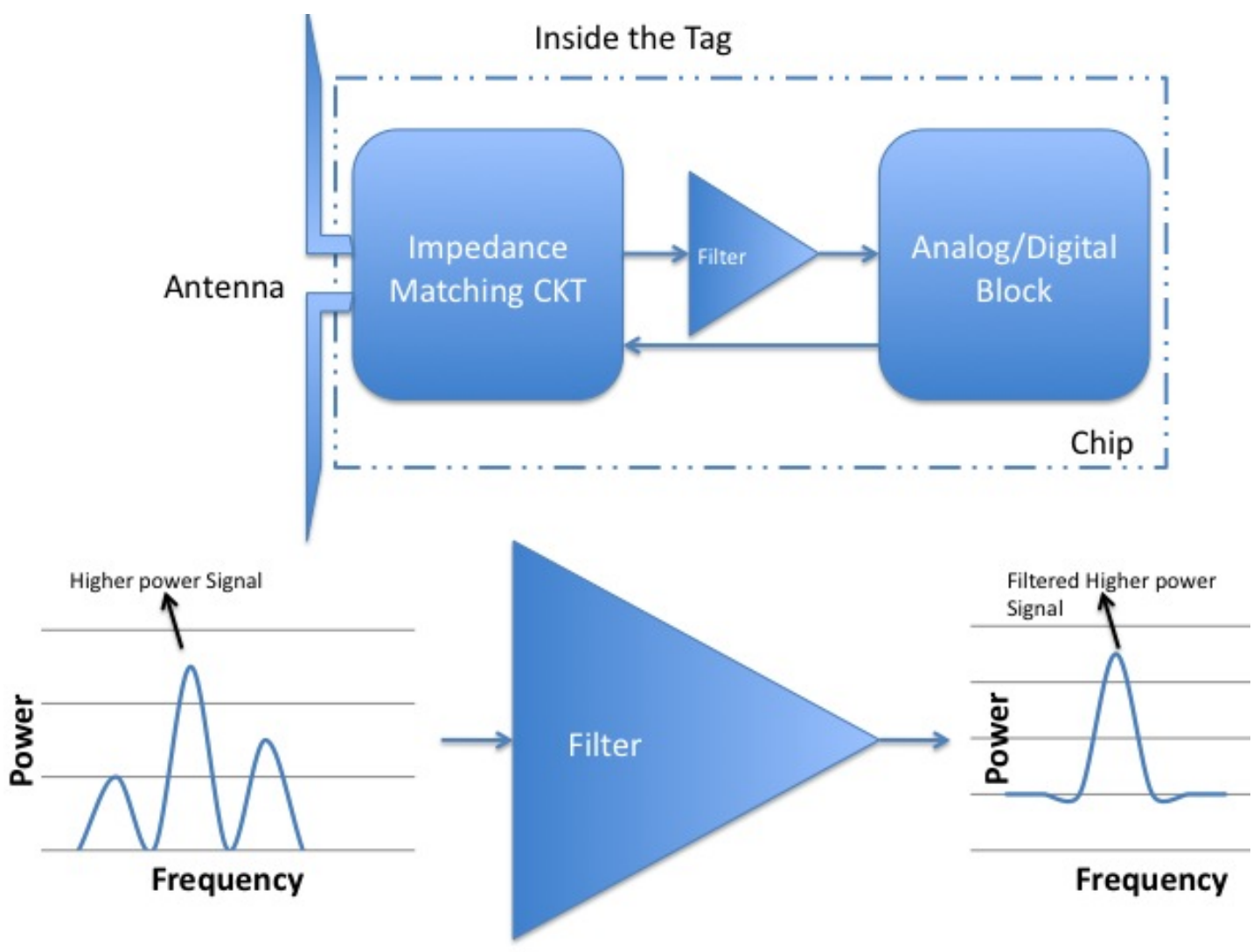

Figure 3.1. Block diagram showing inside of the Tag and its filter.

we show impedance matching circuit, filter and analog/digital block for our understanding. The impedance matching circuit matches the impedance of the antenna. Analog/digital block hold the Tag ID and does all the required processing. Filter block is an important block for our understanding of Tag capture. It filters and selects a high power signal from noise and other signals.

Tags are wide-band receivers, it is important for a Tag to identify at the frequency slot. In case of multiple Reader environment Tags receive many signals in the frequency band 902-928MHz. Readers continuously does frequency hopping looking for which slot the Tags reply. Tags follow a simple technique to choose which Reader to reply to. Tag just identifies the maximum power signal and chooses to reply in 
that channel. So its filter block which identifies higher power signal and filters out all the other signals as shown in the Figure 3.1. This is done simply to avoid collisions, it starts replying in this selected slot and other readers are not communicated with. It is quite obvious to note that now the Tags have locked to this frequency slot and continues to communicate with this Reader provided the Reader maintains the same slot and power level. This may be due to filter capacitors and indictors maintaining the charge and hence filter parameters. This is a Tag capture condition. But FCC part 15 regulations allow the Reader to stay in the slot for a maximum of $400 \mathrm{~ms}$. Hence it depends on the Reader frequency hopping, power ramping etc.

\subsection{Reader Physics}

In this section we will discuss about the communication physics of the Reader pertaining to the Tag capture mentioned in previous section. The Reader comply under FCC part 15.247 for digital modulation systems. Maximum allowable output power is $1 \mathrm{~W}(+30 \mathrm{dBm})$. Also average time of occupancy on any frequency shall not be greater that $0.4 \mathrm{~s}$ within a $20 \mathrm{~s}$ period. Also for frequency hopping if the $20 \mathrm{~dB}$ bandwidth of the hopping channel is less than $250 \mathrm{kHz}$, the Reader shall use at least 50 hopping frequencies. Maximum allowed 20dB bandwidth of the hopping channel is $500 \mathrm{kHz}$.

In practice Readers follow techniques that can provide maximum readability, maximum range and avoid collisions. Readers might follow slot occupancy as show in the Figure 3.2. Here slots refers to the frequency slots occupied during hopping. Freq1-4 represents different instances of frequency hopping. Typically the slot occupancy time might vary from $300 \mathrm{~ms}$ to $20 \mathrm{~ms}$. Readers spending more time in a slot simply means that Reader is reading the Tag continuously in this slot period. Since the readability of a Tag depends on the slot occupancy time, we can say that the 


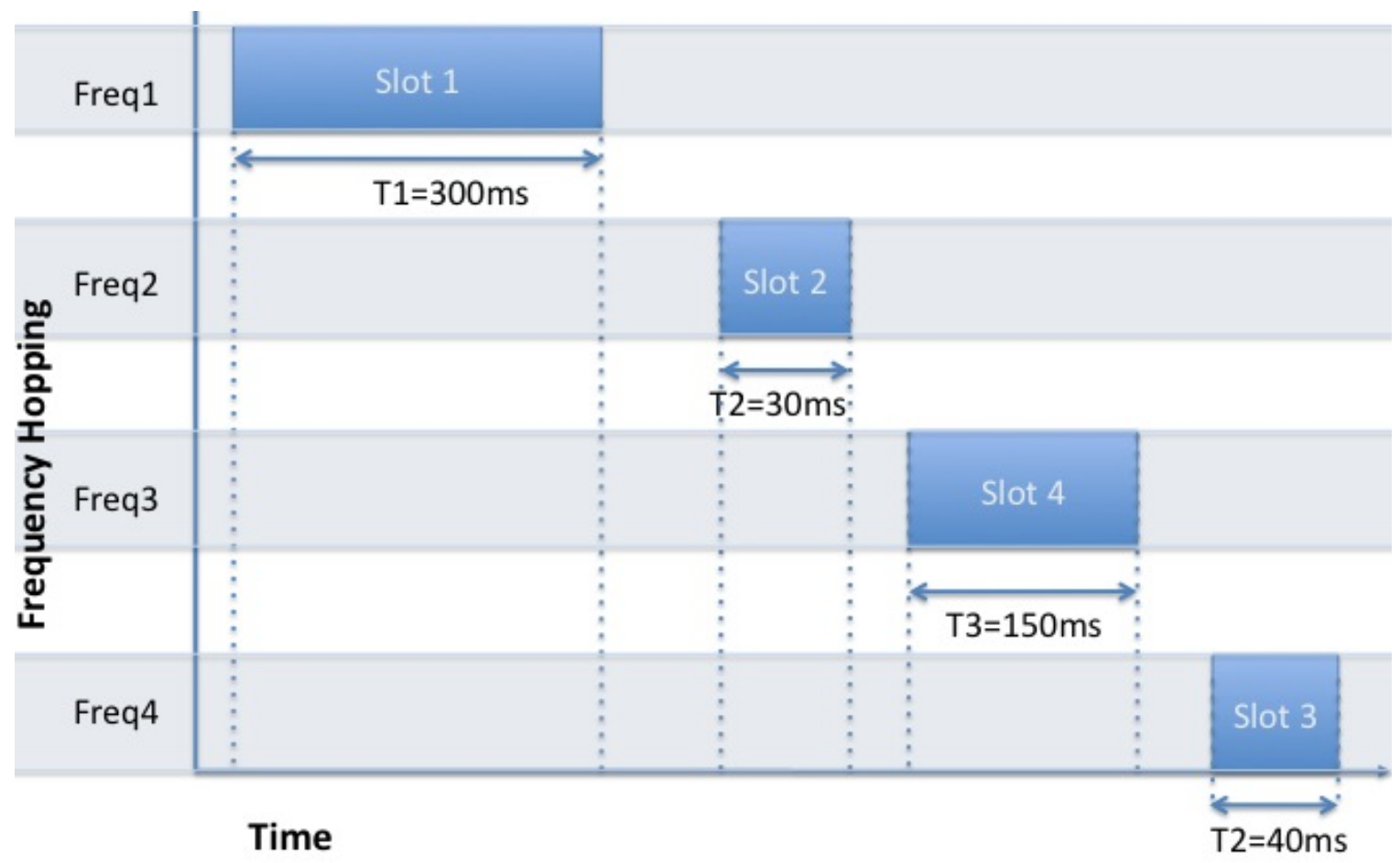

Figure 3.2. Reader frequency hopping and slot occupancy.

Tag capture also depends on the slot occupancy time. During frequency hopping the Reader take time in moving to different slot. This may be due to processing time or the transceiver frequency hopping limitations. Sometime it would be necessary to increase the power when a Reader doesn't detect any Tags in the previous slot. It takes time for processor of the Reader to make necessary changes in power. Due to all these reasons there might be a slight delay for the next slot to start. This might be sufficient for other Readers to capture the Tag. 


\section{CHAPTER 4 \\ ISO 180006-C GENERATION-2 PROTOCOL}

\subsection{Introduction}

A protocol is a language that a machine understands. Protocols are needed in hardware to achieve coordinated action in the presence of complicating factors such as communication channels that impose long and possibly varying delays or deliver messages unreliably or out-of-order, bounded buffers or other scarce resources that may cause deadlocks, and components that have unpredictable delays and other nondeterministic behavior [14]. The protocol used in the design is ISO 180006-C class 1 Gen 2 protocol. This was developed at the Auto-ID center at MIT [3]. This standardized for passive UHF RFID systems for communication at $860 \mathrm{MHz}-960 \mathrm{MHz}$ [3]. The protocol has commands that are used to establish communications between reader and tag. The interrogator modulates the carrier wave $902 \mathrm{MHz}-928 \mathrm{MHz}$ and the following commands are sent. Based on the commands sent the passive RFID tag will backscatter and sends commands by further modulating. The UHF band is separated into various regions around the world. In Europe it is from $865 \mathrm{MHz}-868 \mathrm{MHz}$ with $200 \mathrm{KHz}$ channels, whereas in United States and Canada it is $902 \mathrm{MHz}-928 \mathrm{MHz}$ with $500 \mathrm{KHz}$ channel. [46]

\subsection{Reader Protocol Layers}

The thesis implements a passive UHF RFID Reader, which reads Class-1 identity tags. The Class- 1 tags are a passive backscatter tags with the following minimum features: 
- An object identifier (OID),

- A tag identifier (TID),

- A 'kill' function that permanently disables the Tag,

- Optional password-protected access control, and

- Optional user memory [3].

In case of a reader it should be able to comply on the requirements mentioned in the physical layer, Tag-identification layer and the protocol parameters.

\subsubsection{Physical Layer}

A physical layer is a fundamental layer underlying the logical data structure of a protocol. It is a layer which determines the means the basic hardware does the communication. Here the Gen2 protocol pertaining to the Reader transmitting information to one or more tags is done by modulating the RF carrier using doublesideband amplitude shift keying (DSB-ASK), single-sideband amplitude shift keying (SSB-ASK) or phase-reversal amplitude shift keying (PR-ASK) using pulse-interval encoding (PIE) format. Tags being a passive tag need energy to do communication, so it receives their operating energy from the reader sent modulated RF carrier.

The Tags communicate with the Reader by backscatter-modulating the amplitude and/or phase of the RF carrier signal. FM0 or Miller modulated subcarrier are

the encoding formats used by the Tag verses PIE of the Reader. Since the tags are passive, a half-duplex communication is appropriate.

\subsubsection{Tag-identification Layer}

The Reader needs to manage the Tag population so that it can read multiple tag more efficiently. This is achieved by performing these basic operations: 
- Select: This is a command used by the Reader to select a user specified group of Tags from the population. Which is same as selecting records from a database.

- Inventory: The inventory consists of multiple commands. The reader begins the inventory round by the Query command. This inventory is the core that identify the tags and request the information from the tag like $\mathrm{PC}$, OID, and CRC-16.

- Access: The Access also consists of multiple commands. As the name specifies access consists of set of commands that are used by the reader to access core information from the tag. Usually it comprises of identifying the tag first and then the access operation is done.

\subsection{Reader Protocol Parameters}

In this section we will discuss about the different signal parameters. The detailed discussion of the physical layer in the sections of signaling interface. Which defines frequencies, modulation, data coding, $\mathrm{RF}$ envelope, data rates and the parameters required for $\mathrm{RF}$ communication.

\subsubsection{Operational Frequencies}

A Reader choses to operate at particular frequency range from $860 \mathrm{MHz}$ to $960 \mathrm{MHz}$ determined by the local radio regulations and by the local radio regulations and by the local radio-frequency environment. 


\subsubsection{Reader-to-Tag $(R \Rightarrow T)$ communications}

\subsubsection{Modulation}

As mentioned earlier the reader shall communicate using DSB-ASK, SSB-ASK, or PR-ASK modulation. The reader modulates the RF carrier signal in one of these modulation schemes with PIE encoding to communicate with the tag. The data rate is set by means of the preamble that initiates the round.

\subsubsection{Amplitude Shift Keying (ASK)}

ASK Modulation

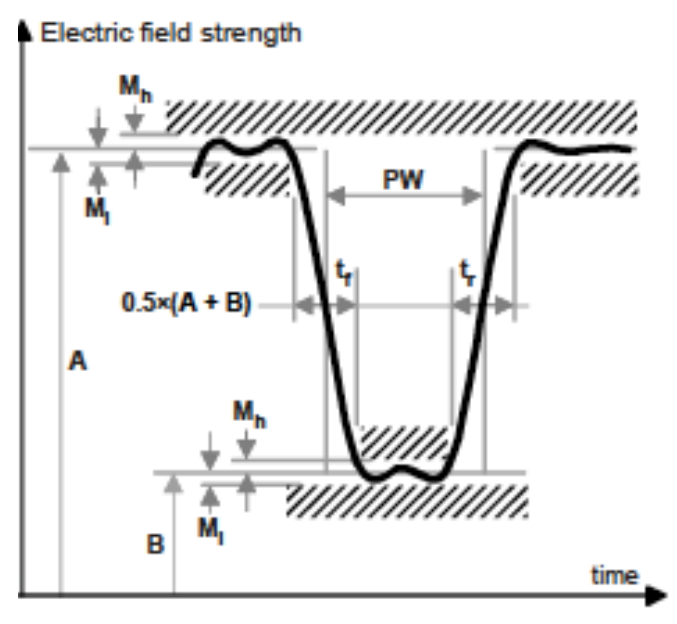

PR-ASK Modulation

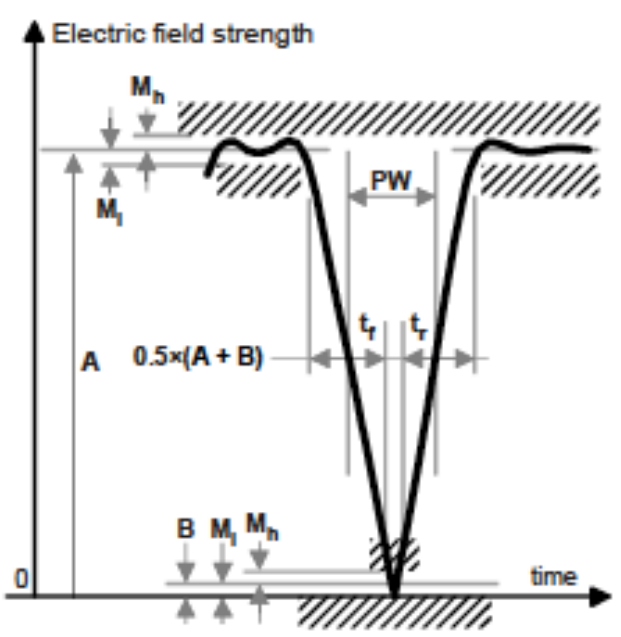

Figure 4.1. Reader to Tag RF modulation envelope [3].

The Figure 4.1 and Table 4.1 shows the modulation parameters for the tari value $6.25 \mu \mathrm{s}$ to $25 \mu \mathrm{s}$. The carrier signal is modulated by varying the envelope of the signal in ASK. The amplitude is varied by encoding the '0's and '1's in the signal. Mostly the signal is having two amplitudes, one low amplitude for a ' 0 ' and high for a '1'. It can change according to the encoding formats. The protocol uses Pulse 
Table 4.1. RF Envelope Parameters [3]

\begin{tabular}{|l|l|l|l|l|l|}
\hline Parameter & Symbol & Minimum & Typical & Maximum & Units \\
\hline Modulation Depth & $(\mathrm{A}-\mathrm{B}) / \mathrm{A}$ & 80 & 90 & 100 & $\%$ \\
\hline RF Envelope Ripple & $\left(M_{h}=M_{l}\right)$ & 0 & & $0.05(\mathrm{~A}-\mathrm{B})$ & $V / m$ \\
\hline RF Envelope Rise Time & $\left(t_{r, 10-90 \%}\right)$ & 0 & & 0.33 Tari & $\mu$ s \\
\hline RF Envelope Fall Time & $\left(t_{f, 10-90 \%}\right)$ & 0 & & 0.33 Tari & $\mu$ s \\
\hline RF Pulsewidth & $\mathrm{PW}$ & MAX $(0.265$ Tari, 2$)$ & & 0.525 Tari & $\mu$ s \\
\hline
\end{tabular}

Interval Modulation (PIE). The On-Off Keying is another type of modulation which the protocol uses. It is similar to ASK where the signal is on/off for a duration creating an on/off envelope.

\subsubsection{Reader power-up/down waveform}

The reader power-up/down RF envelope will comply with the Figure 4.2 and Table 4.2. Here the carrier signal level will rise monotonously from its $10 \%$ level until at least ripple limit $M_{l}$. Similarly when the carrier level fallen below $90 \%$ level for power down, it can fall monotonically until the power-off limit $M_{s}$.

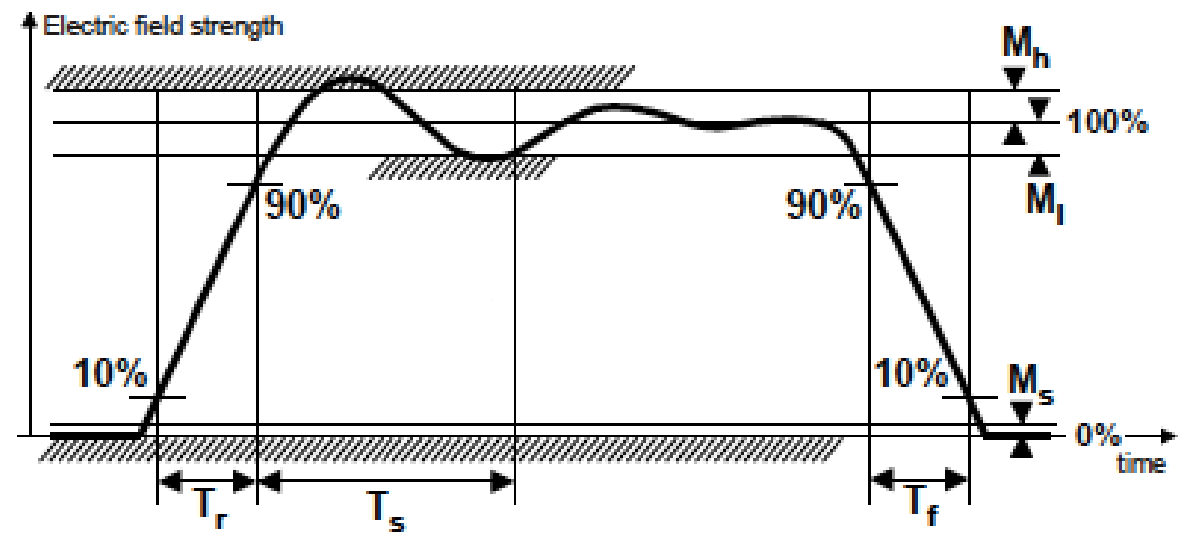

Figure 4.2. Reader power-up/down RF envelope [3]. 
Table 4.2. Reader power-up/down waveform parameters [3]

\begin{tabular}{|l|l|l|l|l|l|}
\hline Parameter & Definition & Minimum & Typical & Maximum & Units \\
\hline$T_{r}$ & Rise time & 1 & & 500 & $\mu \mathrm{s}$ \\
\hline$T_{s}$ & Settling time & & & 1500 & $\mu \mathrm{s}$ \\
\hline$T_{f}$ & Fall time & 1 & & 500 & $\mu \mathrm{s}$ \\
\hline$M_{s}$ & Signal level when OFF & & & 1 & $\%$ full scale \\
\hline$M_{l}$ & Undershot & & & 5 & $\%$ full scale \\
\hline$M_{s}$ & Overshoot & & & 5 & $\%$ full scale \\
\hline
\end{tabular}

Table 4.3. Preferred Tari Values [3]

\begin{tabular}{|l|l|l|}
\hline Tari Value & Tari Tolerance & Spectrum \\
\hline $6.25 \mu \mathrm{sec}$ & $\pm 1 \%$ & $\begin{array}{l}\text { DSB-ASK, SSB- } \\
\text { ASK or PR-ASK }\end{array}$ \\
\hline $12.5 \mu \mathrm{sec}$ & $\pm 1 \%$ & $\begin{array}{l}\text { DSB-ASK, SSB- } \\
\text { ASK or PR-ASK }\end{array}$ \\
\hline $25 \mu \mathrm{sec}$ & $\pm 1 \%$ & $\begin{array}{l}\text { DSB-ASK, SSB- } \\
\text { ASK or PR-ASK }\end{array}$ \\
\hline
\end{tabular}

\subsubsection{Data rates}

The Reader shall communicate with the Tari values as shown in the Table 4.3. With time width values fixed for '0's and '1's with reference to Tari value the data rate changes. The data rate chosen is very important. Some Reader design doesn't allow some data rates. This effects the performance of the system. So selection of a Reader design is also dependent on the data rate. Later will discuss about the selection of a data rate for good architecture design of the reader. The Gen 2 compliant Tari values are shown in the Table 4.3. 


\subsubsection{Data encoding}

The Reader to Tag communication use Pulse Interval Modulation as a data encoding scheme. Tari is the reference time interval for interrogator-to-tag signaling, and is the duration of data-0 [3]. The PIE symbols are as show in the Figure 4.3. Showing the PIE, where the high value is CW at higher amplitude and low values is the CW at lower amplitudes.

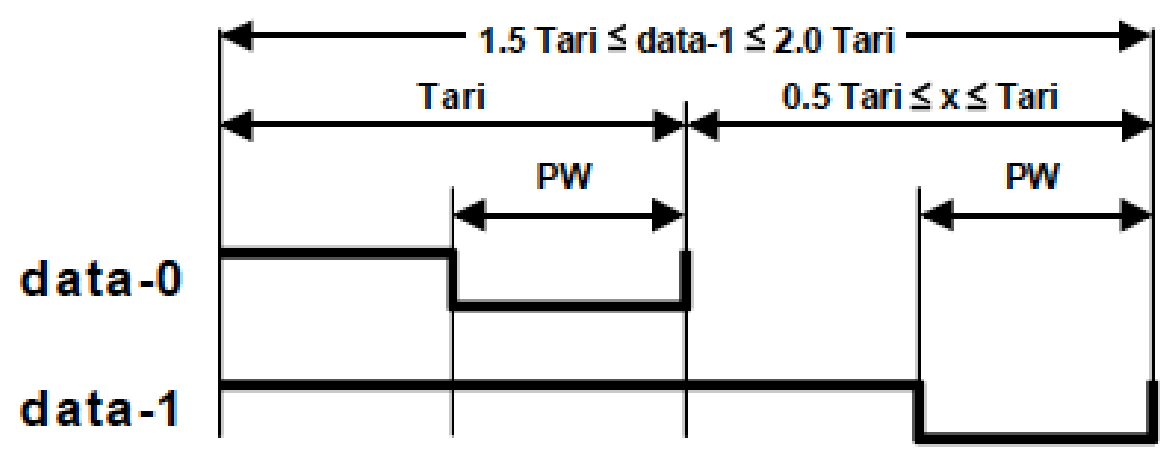

Figure 4.3. PIE symbols [3].

\subsubsection{Reader to Tag Preamble and Frame-sync}

A reader will begin any communication with the Tag with either preamble or a frame-sync. In the inventory commands the Query command is the most important as the reader states with this command. The preamble precedes this query command only. Whereas the frame-sync precedes every other commands.

The preamble and frame-sync is as shown in the Figure 4.4. Preamble consists of a fixed-length delimiter, a data-0 symbol, an $R \Rightarrow T$ calibration (RTcal) symbol, and a $\mathrm{T} \Rightarrow \mathrm{R}$ calibration (TRcal) symbol. Frame-sync is same as preamble expect the last TRcal symbol. 
$\underline{R=>T \text { Preamble }}$

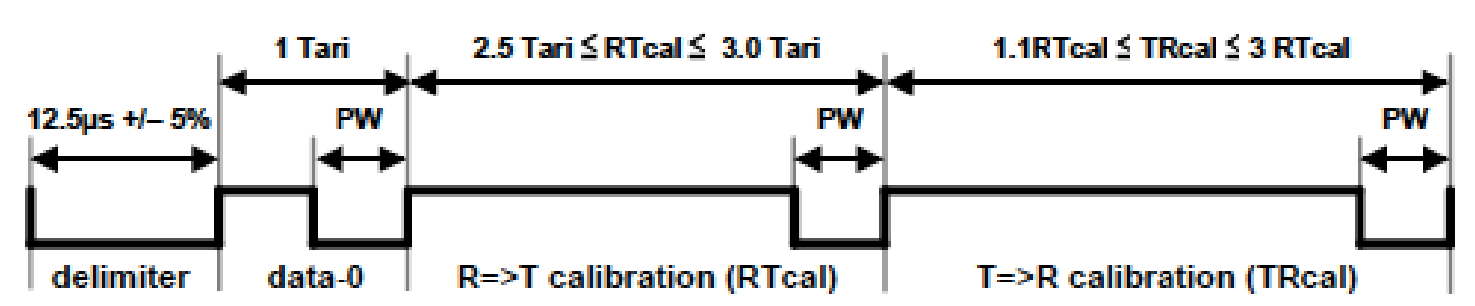

$\underline{R}=>$ T Frame-Sync

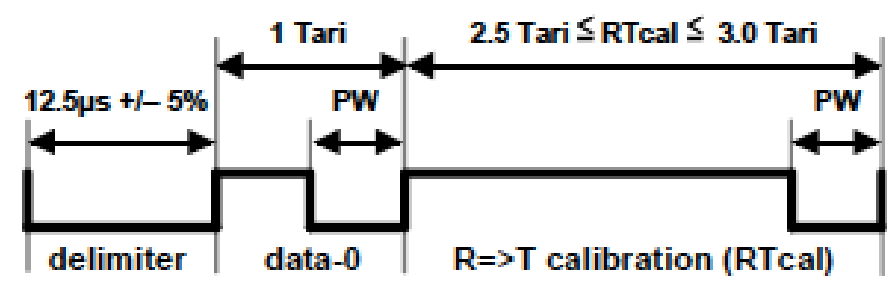

Figure 4.4. The Preamble and Frame-sync [3].

- RTcal: The reader can set the length of RTcal $=0_{\text {length }}+1_{\text {length }}$. So that the tag can measure RTcal and calculate pivot $=\mathrm{RTcal} / 2$. Now the tags will interpret a symbol smaller than pivot as data-0 and longer as data-1. Also the Tag shall consider signals longer than 4 RTcal as bad data. A CW is transmitted for a minimum of 8 RTcal before changing RTcal.

- TRcal: A TRcal length is chosen by the Reader to specify the link frequency(LF). This LF is used by the tag to determine its FM0 data rate. The LF is calculated from the equation 4.1. Where DR is the Divide ratio already known by Tag specified by the manufactrer.

$$
L F=\frac{D R}{T R c a l}
$$




\subsubsection{Frequency-Hopping Spread-Spectrum (FHSS)}

The FHSS signaling of the Reader shall comply on the FCC regulations. It shall comply on the RF envelope shown in the Figure 4.2. So it is important to consider these regulation in the reader design.

\subsubsection{Tag-to-Reader $(\mathrm{T} \Rightarrow \mathrm{R})$ communications}

\subsubsection{Modulation}

Modulation shall be same as reader, as explained earlier in the sections 3.3.2.1 -3.3 .2 .3 .

\subsubsection{Data encoding}

The backscattered data from the Tag shall be encoded by FM0 baseband or Miller modulation of a subcarrier at the data rate.

\section{- FM0 baseband:}

FM0 encoding or the bi-phase space is as shown in the Figure 4.5. The FM0 basis functions showing the data-0 and data-1. And the FM0 generator state diagram labels, $\mathrm{S}_{1}-\mathrm{S}_{4}$, indicating four possible FM0-encoded symbols.

- Miller-modulated subcarrier

Miller encoding is as shown in the Figure 4.6. The FM0 basis functions showing the data-0 and data-1. And the FM0 generator state diagram labels, $\mathrm{S}_{1}-\mathrm{S}_{4}$, indicating four possible Miller-modulated subcarrier symbols. In this encoding the duty cycle of the symbols is measured at output and is between $45 \%$ to 55\%. A Miller encoding signaling shall end with a "dummy" data-1 bit at the end of signal transmission. 

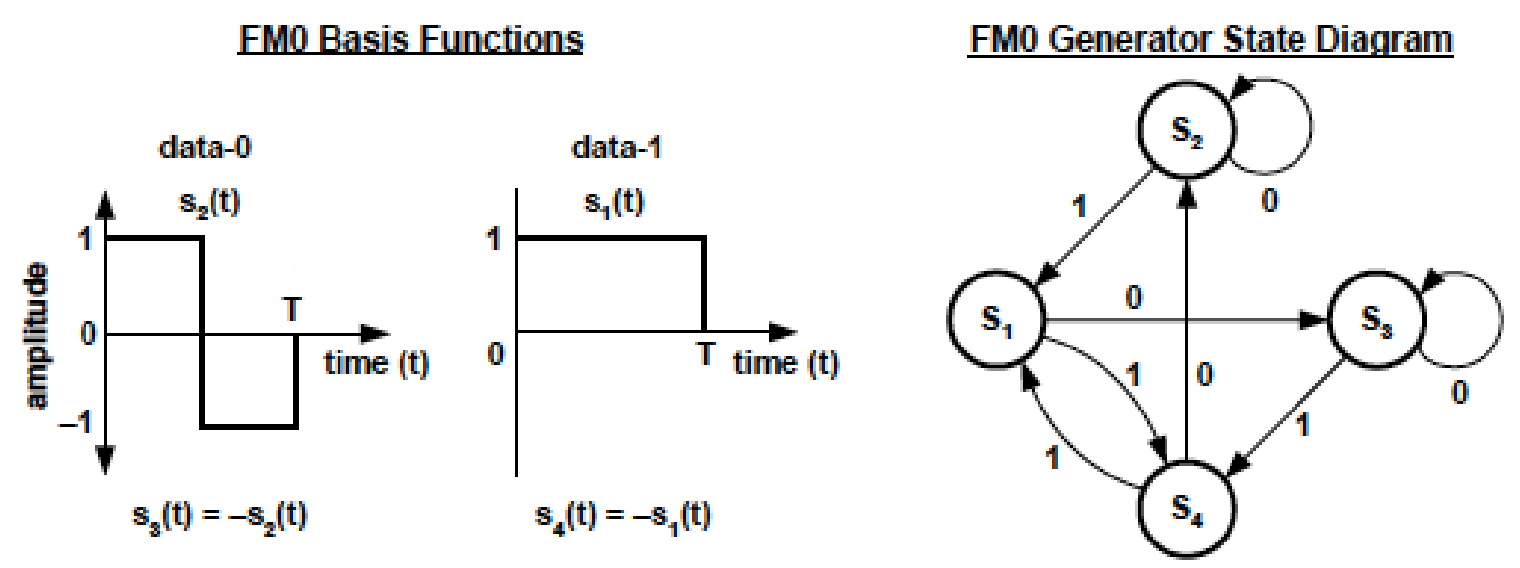

Figure 4.5. FM0 basis functions and generator state diagram [3].
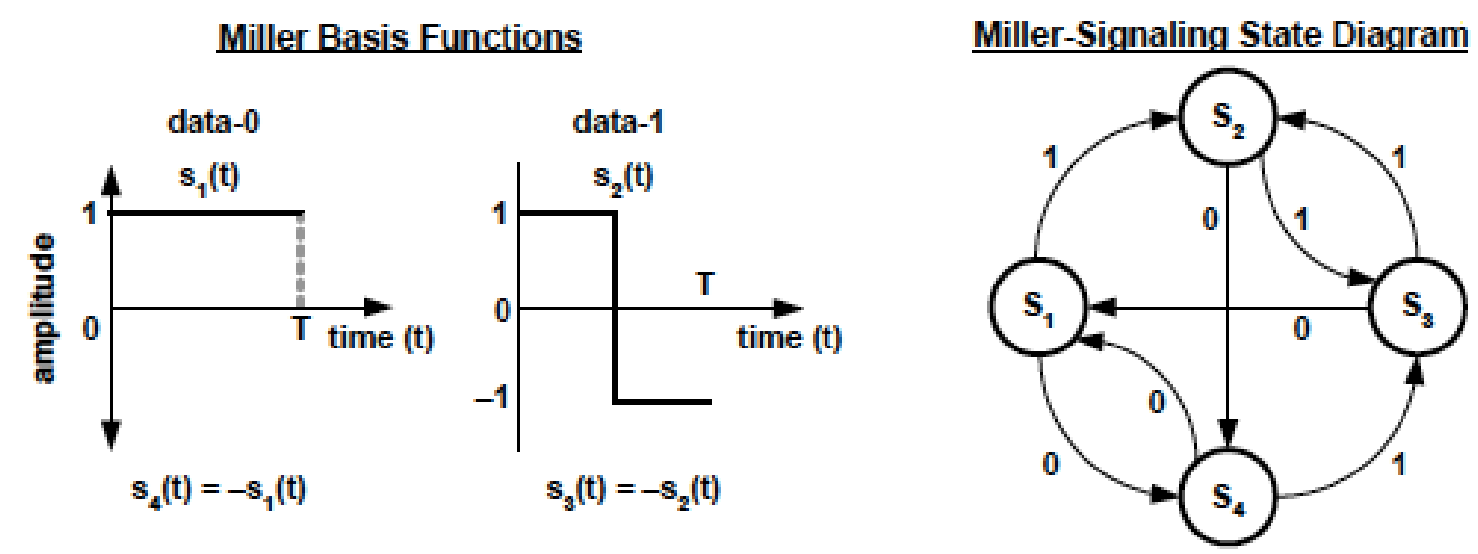

Figure 4.6. Miller basis functions and generator state diagram [3].

\subsubsection{Link timings}

The link timing of the communication between the Reader and Tag is shown in the Figure 4.7. Table 4.4 consists of link timing parameters. A inventory command consists of Query command which initiates the round. The Query command consists of $\mathrm{DR}$ and $\mathrm{M}$ values(which is discussed in later sections); the preamble that precedes 
the Query specifies TRcal. These parameters are used by Tag to determine the back scatter frequency, modulation type and $\mathrm{T} \Rightarrow \mathrm{R}$ data rate.

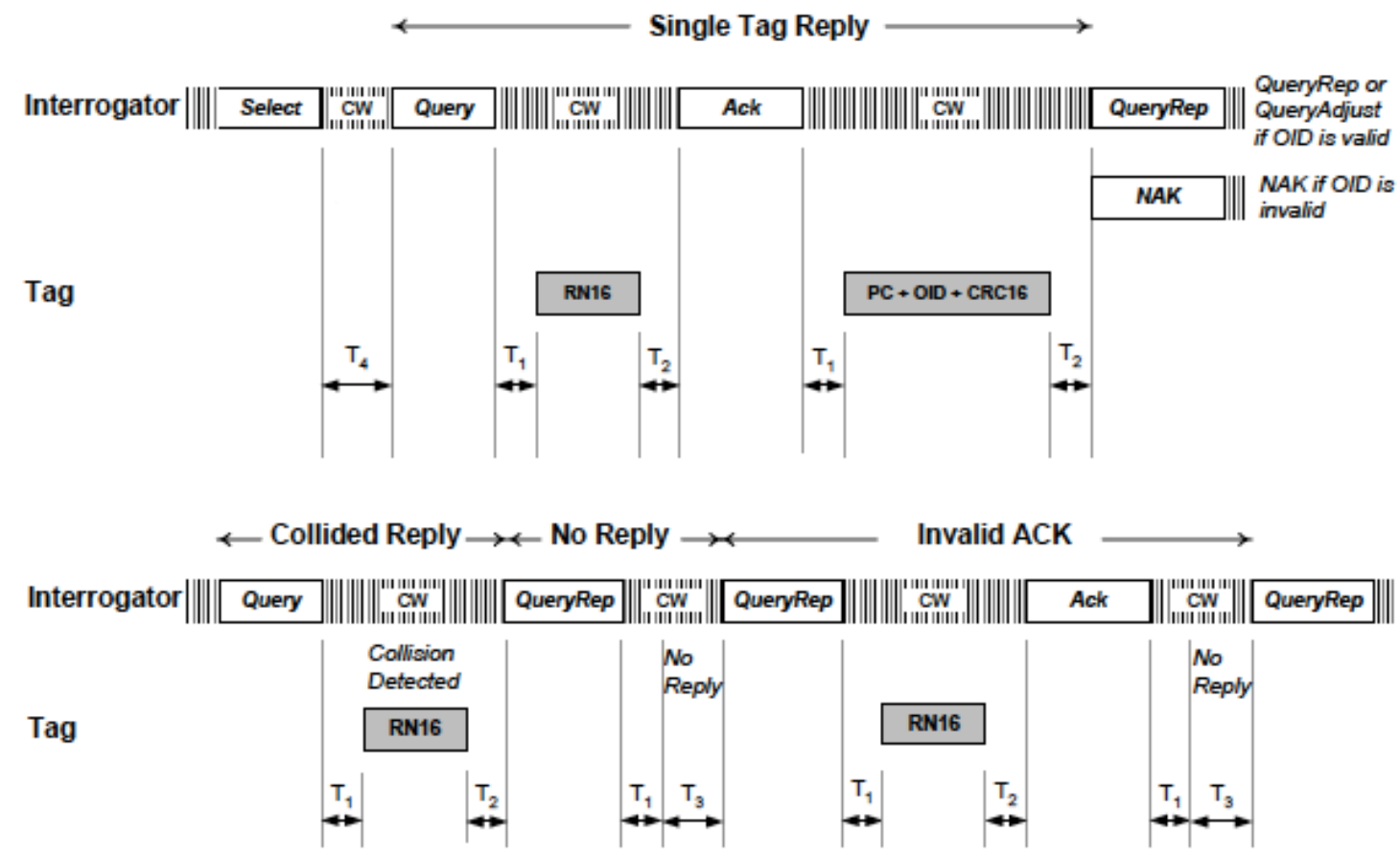

Figure 4.7. Link timing [3].

Inventory round of a single tag reply shown in Figure 4.7 shows the reader transmitting $\mathrm{CW}$ for a duration of $\mathrm{T}_{4}$ which is minimum of $2 \mathrm{RT}$ cal. The Reader shall use a constant $R \Rightarrow T$ link rate, if a change of $R \Rightarrow T$ link rate is required to be changed then a $\mathrm{CW}$ for a minimum of $8 \mathrm{RT}$ cal is transmitted to indicate there is a change in data rate. When a Query is communicated with the tag the Tag sends RN16 after a time $\mathrm{T}_{1}$ as shown. Reader waits for time $\mathrm{T}_{2}$ and sends Ack command. Now the tag reply with a command consisting of PC, OID and CRC16. An QueryRep or QueryAdjust is sent by reader to acknowledge that OID is valid. Else a NAK command is sent. 
Table 4.4. Link timing parameters [3]

\begin{tabular}{|l|l|l|l|l|}
\hline Parameter & Minimum & Typical & Maximum & Description \\
\hline$T_{1}$ & $\begin{array}{l}M A X\left(R T c a l, 10 T_{p r i}\right) \\
\times(1-F T)-2 \mu \mathrm{s}\end{array}$ & $M A X\left(R T c a l, 10 T_{p r i}\right)$ & $\begin{array}{l}M A X\left(R T c a l, 10 T_{p r i}\right) \\
\times(1-F T)+2 \mu \mathrm{s}\end{array}$ & $\begin{array}{l}\text { Time from interrogrator } \\
\text { transmission to tag re- } \\
\text { sponse, measured at an- } \\
\text { tenna terminal. }\end{array}$ \\
\hline$T_{2}$ & $3.0 \mathrm{~T}_{p r i}$ & $20.0 \mathrm{~T}_{p r i}$ & $\begin{array}{l}\text { Time from last falling } \\
\text { edge of the last bit of } \\
\text { the Tag response to first } \\
\text { falling edge of a Reader } \\
\text { signal. }\end{array}$ \\
\hline$T_{3}$ & $0.0 \mathrm{~T}_{p r i}$ & $\begin{array}{l}\text { Time an Reader waits, af- } \\
\text { ter } \mathrm{T}_{1} \text {, before it issues an- } \\
\text { other command }\end{array}$ \\
\hline$T_{4}$ & $2.0 \mathrm{~T}_{p r i}$ & & $\begin{array}{l}\text { Minimum time between } \\
\text { Reader commands }\end{array}$ \\
\hline
\end{tabular}

\subsubsection{Reader Commands and Tag replies}

Reader-to-Tag commands shall have the format shown in the Table 4.5. Each and every command shall have a command code preceding the commands. It is unique to every command. The Table 4.5 indicates the codes and length of the few commands. The Tags shall ignore invalid commands. Following shall be the reasons for the commands to be invalid.

- If the current Tag state is incorrect.

- Command not supported by Tag.

- If the parameters are incorrect.

- If the CRC error is detected.

- An incorrect section is specified.

- Other unrecognized encoding/signaling.

A CRC-5 is 5 bits of length is defined by the polynomial shown in the Equation 4.2. The preset value is 01001 and residue 00000 . 
Table 4.5. Commands [3]

\begin{tabular}{|l|l|l|l|l|}
\hline Command & Code & Length(bits) & Required? & Protection \\
\hline QueryRep & 00 & 4 & Yes & Unique command length \\
\hline ACK & 01 & 18 & Yes & Unique command length \\
\hline Query & 1000 & 22 & Yes & Unique command length and CRC \\
\hline QueryAdjust & 1001 & 9 & Yes & Unique command length \\
\hline Select & 1010 & $>43$ & Yes & CRC-16 \\
\hline NAK & 11000000 & 8 & Yes & Unique command length \\
\hline
\end{tabular}

Table 4.6. Select command [3]

\begin{tabular}{|c|c|c|c|c|c|c|c|c|c|}
\hline & Command & Target & Action & MemBank & Pointer & Length & Mask & Truncate & CRC-16 \\
\hline \# of bits & 4 & 3 & 2 & 2 & EBV & 8 & variable & 1 & 16 \\
\hline Description & 1010 & $\begin{array}{l}\text { 000:S0 } \\
001: S 1 \\
010: S 2 \\
011: S 3 \\
\text { 100:SL } \\
\text { 101:RFU } \\
\text { 110:RFU } \\
\text { 111:RFU }\end{array}$ & $\begin{array}{l}\text { See } \\
\text { Table } 4.7\end{array}$ & $\begin{array}{l}00: \text { RFU } \\
\text { 01:OID } \\
\text { 10:TID } \\
\text { 11:User }\end{array}$ & $\begin{array}{l}\text { Starting } \\
\text { Mask } \\
\text { Address }\end{array}$ & $\begin{array}{l}\text { Mask } \\
\text { Length } \\
\text { (bits) }\end{array}$ & Mask value & $\begin{array}{l}0 \text { :Disable } \\
1 \text { :enable }\end{array}$ & \\
\hline
\end{tabular}

$$
\text { Polynomial }=X^{5}+X^{3}+1
$$

\subsubsection{Select (Mandatory)}

The Select command implemented by the Reader/Tag selects a particular Tag population. The Select command comprises of the following bits as shown in Table 4.6. Based on the Target, Acton, MemBank, Pointer, Length and Mask fields the Tag matching and non-matching criteria is specified. The response to the Action parameters is as shown in Table 4.7 . 
Table 4.7. Tag response to action parameter [3]

\begin{tabular}{|l|l|l|}
\hline Action & Matching & Non-Matching \\
\hline 00 & assert SL or inventoried $\rightarrow$ A & deassert SL or inventoried $\rightarrow$ B \\
\hline 01 & assert SL or inventoried $\rightarrow$ A & do nothing \\
\hline 10 & do nothing & deassert SL or inventoried $\rightarrow$ B \\
\hline 00 & negate SL or $(\mathrm{A} \rightarrow \mathrm{B}, \mathrm{B} \rightarrow \mathrm{A})$ & negate SL or $(\mathrm{A} \rightarrow \mathrm{B}, \mathrm{B} \rightarrow \mathrm{A})$ \\
\hline
\end{tabular}

Table 4.8. Query commands [3]

\begin{tabular}{|c|c|c|c|c|c|c|c|c|c|}
\hline & Command & DR & $\mathbf{M}$ & TRext & Sel & Session & Target & $\mathrm{Q}$ & CRC-16 \\
\hline \# of bits & 4 & 1 & 2 & 1 & 2 & 2 & 1 & 4 & 5 \\
\hline Description & 1000 & $\begin{array}{l}0: \mathrm{DR}=8 \\
1: \mathrm{DR}=64 / 3\end{array}$ & $\begin{array}{l}00: \mathrm{M}=1 \\
01: \mathrm{M}=2 \\
10: \mathrm{M}=4 \\
11: \mathrm{M}=8\end{array}$ & $\begin{array}{ll}0: \text { No } & \text { pilot } \\
\text { tone } & \\
1: \text { Use } & \text { pilot } \\
\text { tone } & \end{array}$ & $\begin{array}{l}00: \text { All } \\
01: \text { All } \\
10: \sim \text { SL } \\
11: \text { SL }\end{array}$ & $\begin{array}{l}00: \mathrm{S} 0 \\
01: \mathrm{S} 1 \\
10: \mathrm{S} 2 \\
11: \mathrm{S} 3\end{array}$ & $\begin{array}{l}0: \mathrm{A} \\
1: \mathrm{B}\end{array}$ & $0-15$ & \\
\hline
\end{tabular}

\subsubsection{Query (Mandatory)}

Query command is the most important command in the inventory round. It initiates and specifies the complete operation of Reader and Tag. This command is extensively used by the Blocking Reader. For any tag to respond query is the trigger command. If a Tag, in response to the Query, draws a zero slot value form its RNG, then its reply to a Query shall be with a RN16 value, otherwise the tag shall remain silent [3]. Table 4.8 shows the bits of the command. The $D R$ is TRcal divide ratio which sets the $\mathrm{T} \Rightarrow \mathrm{R}$ link frequency. $M$ is the cycles per symbol which sets the $\mathrm{T} \Rightarrow \mathrm{R}$ data rate and modulation format. TRext bit is used to determine if pilot tone should be prepended for $\mathrm{T} \Rightarrow \mathrm{R}$ preamble. The Sel bits which tags respond to the Query. The Session determines which session on inventory round is selected. Target selects the inventoried flags A or B. $Q$ determines the number of slots in the round. 
Table 4.9. QueryAdjust command [3]

\begin{tabular}{|c|c|l|l|}
\hline & Command & Session & UpDn \\
\hline \# of bits & 4 & 2 & 3 \\
\hline Description & 1001 & $00: S 0$ & $110: \mathrm{Q}=\mathrm{Q}-1$ \\
& & $01: \mathrm{S} 1$ & $000:$ No change to Q \\
& & $10: \mathrm{S} 2$ & $011: \mathrm{Q}=\mathrm{Q}-1$ \\
& & $11: \mathrm{S} 3$ & \\
\hline
\end{tabular}

Table 4.10. QueryRep command [3]

\begin{tabular}{|c|c|l|}
\hline & Command & Session \\
\hline \# of bits & 2 & 2 \\
\hline Description & 00 & $00: \mathrm{S} 0$ \\
& & $01: \mathrm{S} 1$ \\
& & $10: \mathrm{S} 2$ \\
& & $11: \mathrm{S} 3$ \\
\hline
\end{tabular}

\subsubsection{QueryAdjust (Mandatory)}

A QueryAdjust command is sent by the Reader to adjust the Q parameters of the inventory round only. It consist of the following bits as shown in the Table 4.9. A QueryAdjust is always prepended by a frame-sync. In response to this command the Tag shall send RN16 command. QueryAdjust command consist of command, session and UpDn. The UpDn determines if the Tag shall increment/decrement the $\mathrm{Q}$ or have the $\mathrm{Q}$ value to be same.

\subsubsection{QueryRep(mandatory)}

QueryRep command shall be implemented by the Tag and Reader to decrement the slot value. The Tag shall respond with RN16 if the Slot value is '0'. Table 4.10 shows the command bit of QueryRep. 
Table 4.11. ACK command [3]

\begin{tabular}{|c|c|l|}
\hline & Command & RN \\
\hline \# of bits & 2 & 16 \\
\hline Description & 01 & Echoed RN16 or handle \\
\hline
\end{tabular}

Table 4.12. NAK command [3]

\begin{tabular}{|c|c|}
\hline & Command \\
\hline \# of bits & 8 \\
\hline Description & 11000000 \\
\hline
\end{tabular}

\subsubsection{ACK(mandatory)}

ACK command is a acknowledgment given by the Reader to a signal Tag. It is a command which just replies with the same RN16 sent by the Tag. The command bits are as shown in the Table 4.11 .

\subsubsection{NAK(mandatory)}

A NAK command is implemented by Reader and Tag to command all Tags to return to arbitrate state. Table 4.12 shows the command bits.

\subsection{Summary}

ISO 180006-C GENERATION-2 PROTOCOL is designed for a specific purpose. To have a standardized communication layer for all the Tags. Tag Vender use this extensively now. Implementing this in the Reader requires understanding of timings and different commands. The low cost Reader designs have limited memory. A part of protocol can be implement for the basic operation of the Reader. In the next chapter we will discuss about implementation of this protocol in the Blocking Reader. 


\section{CHAPTER 5}

\section{READER ARCHITECTURE}

\section{$5.1 \quad$ Introduction}

A Typical RFID Reader consists of a Central Processing unit, a RF front-end and server interface as shown in the Figure 5.1.

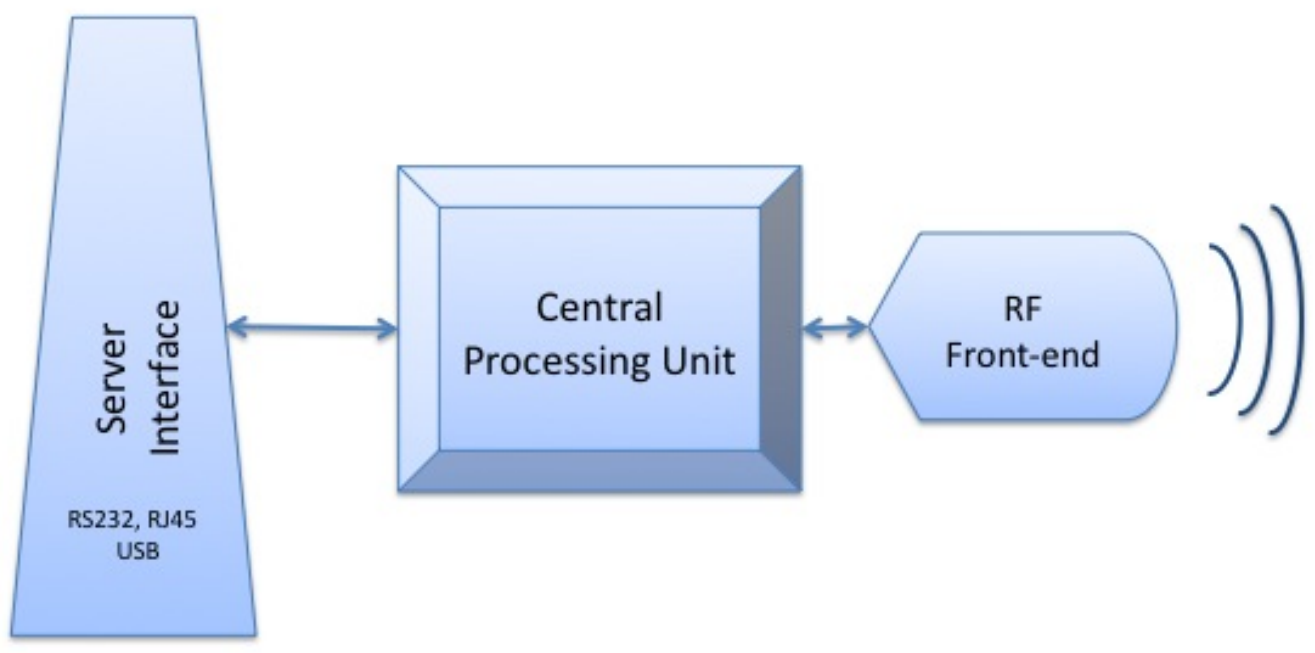

Figure 5.1. Basic functional blocks of a RFID Reader.

RFID Readers come in many designs. The cost of a typical UHF RFID passive Reader varies from $\$ 1,295$ (for Alien ALR-9800) to $\$ 25,000$ (for a Reader test equipment). The UHF Readers available in the market are designed solely for specific applications. Some are designed for performance, some are designed for doing tag 
analysis. But all this Reader systems contain all this basic functional blocks shown in the Figure 5.1.

\subsection{Low-Cost Reader Requirements}

Research is still going to come up with a low cost RFID systems so that it can be extensively used in many applications. The RFID Reader Tag technology replaces the barcode technology. Barcodes and the barcode readers costs a lot less compared to a RFID tag and reader. Currently a RFID passive tag is greater than 8 cents and a typical low cost Reader is about $\$ 200-\$ 500$. But a barcode reader is as low as $\$ 18$. So it is important to have a Ultra low-cost Reader and Tag. A lot of research is going on on designing a low cost UHF passive tags. The Auto-ID Center is developing low-cost radio frequency identification (RFID) based systems with the initial application as next generation bar-codes[45]. Based on the assumptions on the deployment of RFID technology the estimated cost of a passive tag in 10 years would be $\$ 0.02$ [32]. So a 0.01cent tag and 10 dollar reader can change the use of RFID systems. And we could see its deployment in many fields and applications.

\subsection{Possible Low-Cost Reader Architectures}

The design presented in this paper has the following design constraints

- The cost the Reader design should be as low as $30 \$$.

- The Reader should comply on Gen 2 protocol.

- Reader functionality should support read/write information to tags.

- Radiated power should not exceed $1 \mathrm{~W}$.

- Achievable read of 100 tags/sec. 
Each objective has its own challenges. To reduce the cost of the reader we have to compromise on different parameters like performance, read rate, read range etc. The performance of this reader will be less compared to other readers. But the reason for this design is the use of this reader in an application where there is no requirement of high processing readers, as discussed earlier. On research there were lots of designs that were feasible. By knowing the functionalities of the blocks, the design is put together. This level of design will reduce the cost of the design, knowing the cost of each and every block. Achieving the first design constraint is very important, this requires the knowledge of other parameters also. Parameters like power, type of modulation, frequency, protocol etc should be considered. Some of the low cost basic designs are shown.

\subsubsection{Duo-PIC separate TX-RX design}

In this design the transmitted and received signal are separately processed with separate processing units as shown in the Figure 5.2. The two processing unit com-

municate with each other through the data bus. So basically this design has two modules. The transmitter module and the receiver module. The transmitter module consists of a processor, transmitter, impedance matching circuit and an antenna. The receiver module consists of a processor, receiver, impedance matching circuit and an antenna. Here the two modules are isolated in analog domain and communicates each other digitally. When looked at this design we might think there are unnecessary modules. But when a low cost processor is used, it is cheap to dedicate a processor to one function. Here a low cost low functional microprocessor can be used and can be dedicated to one function like transmit/receive. The Gen 2 protocol can be written in such a way that the transmitter/receiver module will only transmit/receive and communicate each other using interrupts. The transmitter and receiver can be so 
chosen to work only for the $902-928 \mathrm{MHz}$ ranges. Therefore it reduces the cost on the impedance matching circuit. The Table 5.1 shows set of components that can be used. It also shows the total cost of the Reader.

Advantages of this design are:

- Simple Design.

- Easy to implement the Gen 2 protocol.

- Can further reduce the cost by using low cost IC's.

- Eliminates the requirement of circulator.

Disadvantages of this design are:

- Difficult to find IC's with specific functionality, mostly RF IC's.

- Parallel processing, difficult to handle timings.

- Isolation of antennas. 


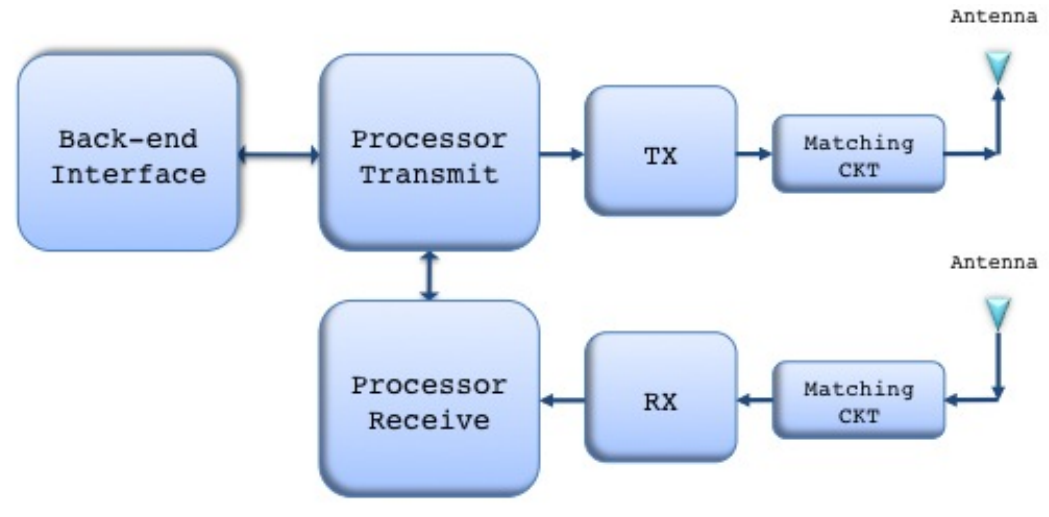

Figure 5.2. Architecture of Duo-PIC separate TX-RX design.

Table 5.1. Duo-PIC separate TX-RX design: parts and cost

\begin{tabular}{|l|l|l|}
\hline Components & \multicolumn{1}{|c|}{ Type of IC/CKT } & Cost(\$) \\
\hline Processor & C8051F320 & $3.16 \times 2$ \\
\hline Transmitter & MTX-103-915DR-B & 9.2048 \\
\hline Receiver & MRX-005-915DR-B & 8.98520 \\
\hline $\begin{array}{l}\text { Ethernet controller } \\
\text { and Connector }\end{array}$ & $\begin{array}{l}\text { ENC28J60/SO-ND } \\
\text { and 609-1059-2-ND }\end{array}$ & 3.6 .9072 \\
\hline $\begin{array}{l}\text { Antenna } \\
\text { Monopole) }\end{array}$ & 509-ANT-915-06A & $7.0 \times 2$ \\
\hline Matching CKT & $\begin{array}{l}\text { Discrete Balun and } \\
\text { Components }\end{array}$ & 4 \\
\hline $\begin{array}{l}\text { Board and other } \\
\text { miscellaneous }\end{array}$ & 2 Layer PCB & 15 \\
\hline \multicolumn{2}{|l|}{ Total Cost } & $\mathbf{6 2 . 0 1 7 2}$ \\
\hline
\end{tabular}




\subsubsection{Duo-PIC separate TX-RX uni-antenna design}

The Duo-PIC separate TX-RX uni-antenna design is an improvement over the Duo-PIC separate TX-RX design. Considering the advantages and disadvantages the Duo-PIC separate TX-RX design can be improved. The Duo-PIC separate TX-RX uni-antenna design is as shown in the Figure 5.3. Architecture of Duo-PIC separate TX-RX uni-antenna design uses a circulator which can eliminate the problem of isolation of two antennas. Further more the impedance matching circuit can be clubbed to single circuit reducing the cost of the design.

This circuit will also have other disadvantages adding to the Duo-PIC separate TX-RX design like

- The design of the circulator for UHF frequencies is bit difficult.

- Cost of the circulator increases design cost.

- Using one antenna reduces the power transmitted to passive tags.

The Table 5.2 shows list of components and its costs. Here you can see the cost of the circulator is potentially increases the cost of the overall design. The total cost of this design considering we use this components is $\$ 77.71$.

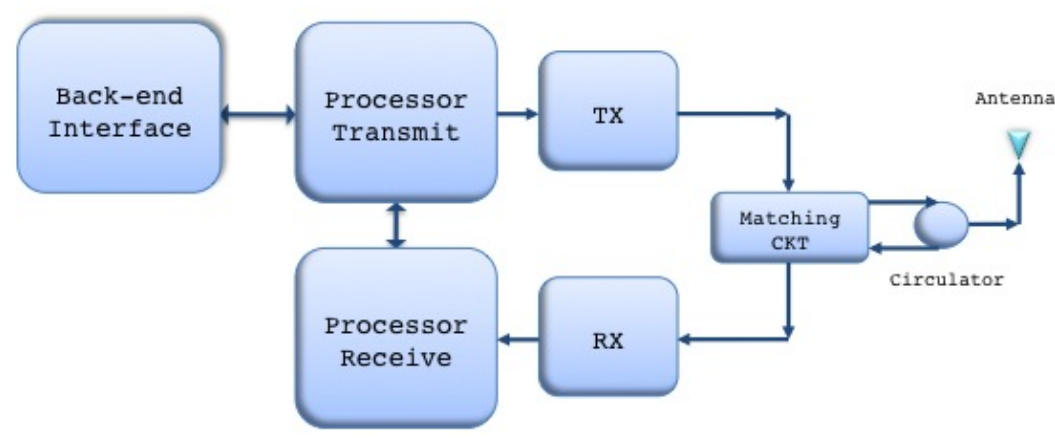

Figure 5.3. Architecture of Duo-PIC separate TX-RX uni-antenna design. 
Table 5.2. Duo-PIC separate TX-RX uni-antenna design: parts and cost

\begin{tabular}{|c|c|c|}
\hline Components & Type of IC/CKT & $\operatorname{Cost}(\$)$ \\
\hline Processor & C8051F320 & $3.16 \times 2$ \\
\hline Transmitter & MTX-103-915DR-B & 9.2048 \\
\hline Receiver & MRX-005-915DR-B & 8.98520 \\
\hline $\begin{array}{l}\text { Ethernet controller } \\
\text { and Connector }\end{array}$ & $\begin{array}{l}\text { ENC28J60/SO-ND } \\
\text { and 609-1059-2-ND }\end{array}$ & $\begin{array}{ll}3.6 & + \\
0.9072 & \end{array}$ \\
\hline $\begin{array}{l}\text { Antenna } \\
\text { (Monopole) }\end{array}$ & 509-ANT-915-06A & 7.0 \\
\hline Matching CKT & $\begin{array}{l}\text { Discrete Balun and } \\
\text { Components }\end{array}$ & 4 \\
\hline $\begin{array}{l}\text { Board and other } \\
\text { miscellaneous }\end{array}$ & 2 Layer PCB & 15 \\
\hline Circulator & SS-SC24A & 22.7 \\
\hline \multicolumn{2}{|c|}{ Total Cost } & 77.7172 \\
\hline
\end{tabular}

\subsubsection{Uni-PIC separate TX-RX design}

In each and every design there is step-wise improvements this will eventually indicate how the final design is come up. The architectural design is as shown in the Figure 5.4. In this design a good performance processor is used with both transmitter and receiver circuit to be different. Here the problem of parallel processing can be eliminated. Also it eliminates the requirement of the other processor reducing the cost. But proper choice of a 16-bit processor like a micro-controller should be used. Disadvantages are same as Duo-PIC separate TX-RX design. 


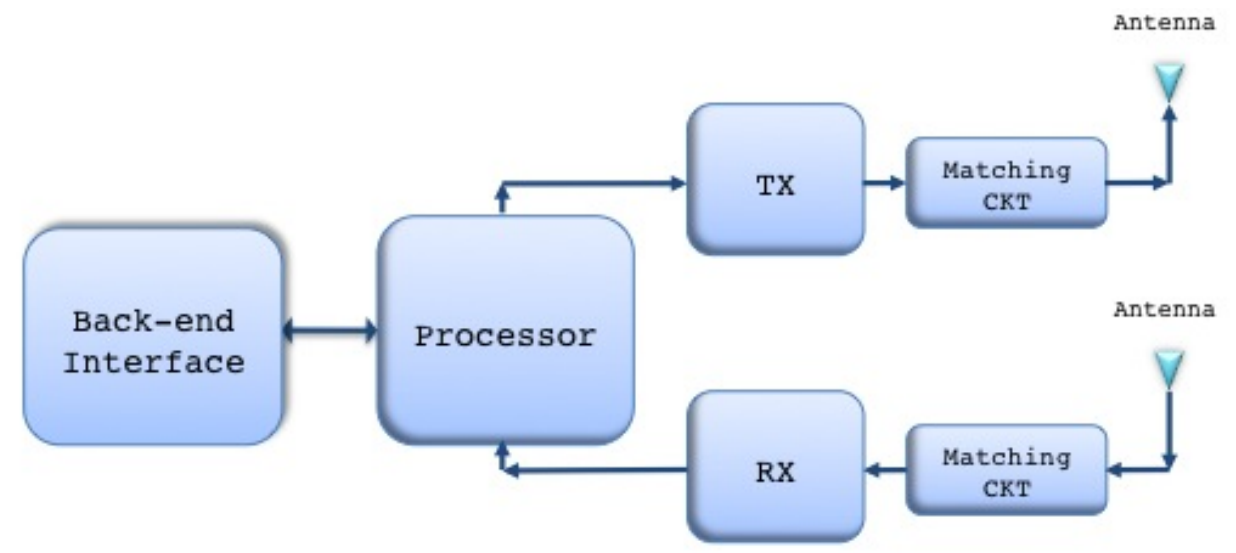

Figure 5.4. Architecture of Uni-PIC separate TX-RX design.

Table 5.3. Uni-PIC separate TX-RX design: parts and cost

\begin{tabular}{|l|l|l|}
\hline Components & \multicolumn{1}{|c|}{ Type of IC/CKT } & Cost(\$) \\
\hline Processor & C8051F320 & 3.16 \\
\hline Transmitter & MTX-103-915DR-B & 9.2048 \\
\hline Receiver & MRX-005-915DR-B & 8.98520 \\
\hline $\begin{array}{l}\text { Ethernet controller } \\
\text { and Connector }\end{array}$ & $\begin{array}{l}\text { ENC28J60/SO-ND } \\
\text { and 609-1059-2-ND }\end{array}$ & 3.6 \\
\hline $\begin{array}{l}\text { Antenna } \\
\text { (Monopole) }\end{array}$ & $509-9072$ \\
\hline $\begin{array}{l}\text { Matching CKT } \\
\text { Board and other } \\
\text { miscellaneous }\end{array}$ & $\begin{array}{l}\text { Discrete Balun and } \\
\text { Components }\end{array}$ & $4.0 \times 2$ \\
\hline \multicolumn{2}{|l|}{ Total Cost PCB } & 15 \\
\hline
\end{tabular}

\subsubsection{Uni-PIC separate TX-RX Uni-antenna design}

In case of this design it is an improvement over Uni-PIC separate TX-RX design like that of transition from Duo-PIC separate TX-RX design to Duo-PIC separate TX-RX uni-antenna design. This design has advantages of elimination of parallel processing and elimination of two antennas topology with no isolation problems. Some 
of the advantages like reduced cots by eliminating one antenna and a processor. But also includes complexity of design with the circulator.

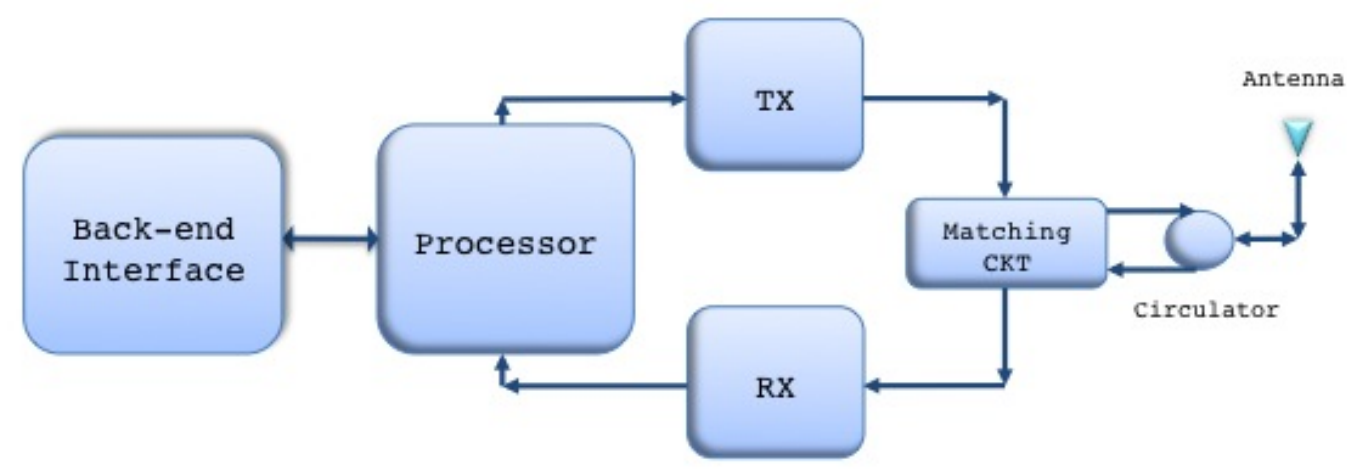

Figure 5.5. Architecture of Uni-PIC separate TX-RX Uni-antenna design.

Table 5.4. Uni-PIC separate TX-RX Uni-antenna design: parts and cost

\begin{tabular}{|l|l|l|}
\hline Components & \multicolumn{1}{|c|}{ Type of IC/CKT } & Cost(\$) \\
\hline Processor & C8051F320 & 3.16 \\
\hline Transmitter & MTX-103-915DR-B & 9.2048 \\
\hline Receiver & MRX-005-915DR-B & 8.98520 \\
\hline $\begin{array}{l}\text { Ethernet controller } \\
\text { and Connector }\end{array}$ & $\begin{array}{l}\text { ENC28J60/SO-ND } \\
\text { and 609-1059-2-ND }\end{array}$ & $\begin{array}{l}3.6 \\
0.9072\end{array}$ \\
\hline $\begin{array}{l}\text { Antenna } \\
\text { (Monopole) }\end{array}$ & 509-ANT-915-06A & 7.0 \\
\hline Matching CKT & $\begin{array}{l}\text { Discrete Balun and } \\
\text { Components }\end{array}$ & 4 \\
\hline $\begin{array}{l}\text { Board and other } \\
\text { miscellaneous }\end{array}$ & 2 Layer PCB & 15 \\
\hline Circulator Total Cost & 22.7 \\
\hline \multicolumn{2}{|l|}{ SS-SC24A } \\
\hline
\end{tabular}




\subsubsection{Single processor transceiver design}

This Single processor transceiver design as shown in the Figure 5.6 can be said as a simplest design. In this design there is a powerful PIC memory sufficient to hold the protocol. A Transceiver that does both transmit and receive of RF signals. The choice of a transceiver IC is very important for this design. And should be able to comply on all the parameters discussed earlier. Again the processor used here should be a 16-bit micro-controller to handle the transceiver. We also note that there is no need for the circulator in this design as the transceiver can do half duplex communication. There is no problem of isolation in this circuit as the design has one antenna.

One disadvantages of this design is it can mostly be used in low power applications, hence the range decreases. A passive RFID Tag uses power from the reader for communication so it is required for the Reader to switch between transmit and receive quickly. The power constraints can be over come by a simple amplifier circuit.

On research and careful consideration this architecture is considered for the design of the Passive UHF RFID Reader in this paper. Important reasons being the simplest design and low cost.

Let us see how the Single processor transceiver design can comply on the design constraints. Cost of the total design should not exceed $30 \$$. The cost discretion is as shown in the Table 5.5 below. From the table we can see that the cost is complied with less that $30 \$$. The next constraint was to see if the design could comply on the Gen2 protocol. Here the transceiver and the RF circuit used should be able to comply on the protocol. CC1101 is the transceiver used in this design this IC is design by Chip Con. The description of the IC CC1101 is as mentioned below. On research this IC was considered as a better option for this design. The next constraint was the on supporting read/write of the tags. This shouldnt be a problem with the 
80C51F320 micro-controller being a programmable which can be programmed to do these functions. The output power of the CC1101 can be programmable up to +10 $\mathrm{dBm}$ making a way below the $1 \mathrm{~W}$ power, it supports for connection of an amplifier to boost the power. So this design complies on the design constraints.

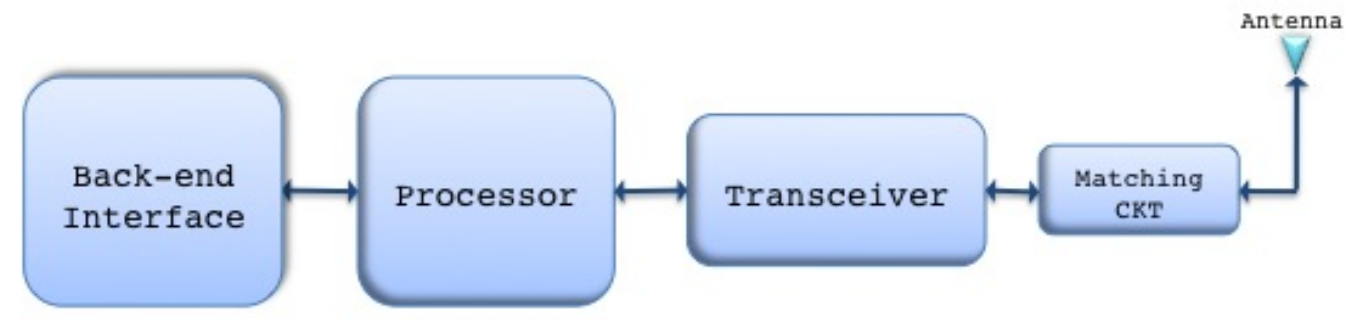

Figure 5.6. Architecture of single processor transceiver .

Table 5.5. List of Parts and Cost for Single processor transceiver design

\begin{tabular}{|l|l|l|}
\hline Components & Type of IC/CKT & Cost(\$) \\
\hline Processor & C8051F320 & 3.16 \\
\hline Transceiver & CC1101 & 2.55 \\
\hline $\begin{array}{l}\text { Antenna } \\
\text { (Monopole) }\end{array}$ & $509-A N T-915-06 \mathrm{~A}$ & 7.0 \\
\hline $\begin{array}{l}\text { Matching CKT } \\
\text { Board and other } \\
\text { miscellaneous }\end{array}$ & $\begin{array}{l}\text { Discrete Balun and } \\
\text { Components }\end{array}$ & 4 \\
\hline \multicolumn{2}{|c|}{ 2 Layer PCB } & 15 \\
\hline \multicolumn{2}{|c|}{ Total Cost } & $\mathbf{3 1 . 7 1}$ \\
\hline
\end{tabular}


Table 5.6. Cost comparison of different Reader designs

\begin{tabular}{|l|l|}
\hline Design & Cost \\
\hline Duo-PIC separate TX-RX design & 62.0172 \\
\hline Duo-PIC separate TX-RX uni-antenna design & 77.7172 \\
\hline Uni-PIC separate TX-RX design & 58.8572 \\
\hline Uni-PIC separate TX-RX Uni-antenna design & 74.5572 \\
\hline Single processor transceiver design & 31.71 \\
\hline
\end{tabular}

\subsection{Amplifier}

An amplifier is important in Single processor transceiver design as the maximum output power that can be achievable with CC1101 transceiver is $+10 \mathrm{dBm}$. The protocol allows a maximum power of $+30 \mathrm{dBm}[3]$. So we could use an external amplifier that can amplify the signal. Some advantages of using amplifier are

- Will increase read range.

- Keeps the Tags in active state.

- Gives an opportunity to design a simple reader with no limit to power.

Some disadvantages of using amplifier are

- Increased cost.

- Will also amplifies the noise in the channel.

- Some loss in data due to inbuilt filters.

\subsection{Summary}

A low cost UHF RFID Reader can be used in different applications. One of the important application we intend to use this design is for a Blocking Reader. Now a low cost Blocking Reader can be used to preserve privacy of the Tags. Later in this thesis we discuss how this design is implemented and show the performance of the working Blocking Reader. 


\section{CHAPTER 6 \\ IMPLEMENTATION}

\subsection{Introduction}

The Blocking Reader uses the Reader design shown in Figure 5.6. This looks like a simple design but implementation of this requires proper selection of the IC's that satisfies the basic requirements.

\subsection{Circuit Diagram}

\subsubsection{Processor}

The processor used in this design is C8051F320. The processor is having a 16Kbytes of Flash sufficient to program a Blocking Reader. It supports all USB and UART modes which provides interface to the server. 25/21 digital I/O ports and 16bit programmable counter array which can be used to interface with the transceiver. The processor is high speed with pipelined instruction architecture executing $70 \%$ of instructions in 1 or 2 clock cycles provides a fast programming of transceiver IC. It is having a throughput of $25 \mathrm{MIPS}$ with a clock of $25 \mathrm{MHz}$. It comes in the packaging of 32-pin LQFP. The $\mu$ Controller connections are as shown in the Figure 6.6.

\subsubsection{Programming}

The $8051 \mu \mathrm{C}$ chip include on-chip Silicon Labs 2-Wire (C2) debug circuitry. It allows a non-intrusive, full speed, in-circuit debugging. A full access to 16KB flash, 2304 bytes of on chip memory and registers. Debugging logic supports setting break

point, single stepping, run and halt commands. The thesis use a Keil $\mu$ Vision 3 for 
writing the code in assembly, C and Hex formats. The $8051 \mu \mathrm{C}$ connected to USB is used to dump the program.

\subsubsection{USB and UART}

The $\mu$ Controller chip has SMBus $/ I^{2}$ C,Enhanced UART, and Enhanced SPI se-

rial interfaces are implemented in hardware. The presence of Universal Serial Bus (USB) function controller with eight flexible endpoint pipes, integrated transceiver and have a RAM of $1 \mathrm{~K}$ FIFO. The Figure 6.6 shows the connection to a USB connector. Pin 4,5 are $\mathrm{D}+$ and $\mathrm{D}$ - of the $\mu \mathrm{C}$ chip is connected to $\mathrm{D}+$ and $\mathrm{D}-$ of the USB connector. Pin 8 of $\mu \mathrm{C}$ chip is the $V_{\text {bus }}$ which is connected to $V_{\text {bus }}$ of $\mu \mathrm{C}$ chip.

\subsubsection{Transceiver connections}

The $\mu$ Controller chip programs the transceiver controlling the RF front end. Port 0 is connected to the transceiver. The connections are as shown in the Figure 6.6. The $V_{\text {ref }}$ pin $27\left(\mathrm{P}_{0.7}\right)$ of $\mu$ Controller chip is connected to the $\mathrm{GD}_{2}$ of the transceiver. Similarly pin $28\left(\mathrm{P}_{0.6}\right)$ to $\mathrm{GD}_{0}$, pin $31\left(\mathrm{P}_{0.3}\right)$ to $\mathrm{CS}_{n}$, pin $32\left(\mathrm{P}_{0.2}\right)$ to $\mathrm{S}_{1}$ and pin $2\left(\mathrm{P}_{0.0}\right)$ to Sclk of the transceiver respectively. The transceiver allows serial programming, it contains various configuration,status and control registers which are accessible. The configuration is called 4-wire serial configuration and data transfer which is later discussed in further sections.

\subsubsection{Transceiver CC1101}

The Blocking Reader uses a transceiver chip from Chipcon (later bought by TI) know as CC1101. CC1101 is a wonderful low-cost low-power sub-1GHz RF transceiver. It is not solely designed for a RFID purpose like a TI $100 \$$ Reader chip. But the cost and functionality of the chip is very impressive. 
Some Analog and Digital Features of CC1101 that satisfy the design constraints of the UHF RFID Reader are

- Operates at 315/433/868/915 MHz ISM/SRD. Blocking Reader requires 915 $\mathrm{MHz}$.

- Extended frequency band 779-928 MHz.

- Allows OOK and flexible ASK shaping.

- Suitable for frequency hopping with $90 \mu$ s settling time.

- Programmable data rate from 1.2 to $500 \mathrm{kBaud}$. Blocking Reader requires 80 kBaud.

- Programmable output power up to $+10 \mathrm{dBm}$. FCC allows up-to $+30 \mathrm{dBm}$ for RFID Readers.

- In built Packet Handling, supports easy programming of Reader commands.

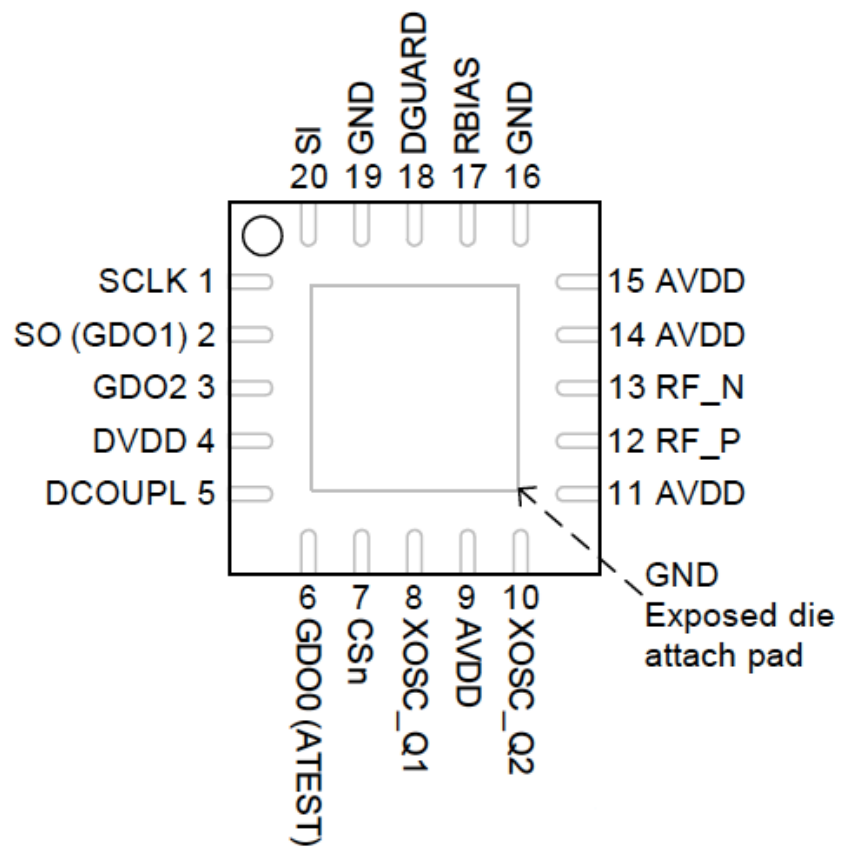

Figure 6.1. Pinout top view [1] . 
The transceiver Pin Configuration is shown in the Figure 6.1. Description of some important pins of the chip are

- SCLK: It is a digital input for serial configuration interface, clock input.

- S0(GDO1): It is a digital output for serial configuration interface, data output. and can be used as optional output pin when CSn is high.

- GDO2: Its is a digital output pin generally used for test signals, FIFO status signals, clear channel indicator, clock output, down-divided from XOSC and serial output RX data

- GDO0(ATEST): It is a digital I/O pin. Generally used for outputting test signals, FIFO status signals, clear channel indicator, clock output, down-divided from XOSC and serial RX/TX data.

- CSn: It is a digital input used for chip selection and serial configuration interface.

- $\mathbf{R F} \_\mathbf{P}$ : It is an RF I/O pin, a positive terminal for RF I/P signal to LNA and $\mathrm{O} / \mathrm{P}$ signal from PA.

- $\mathbf{R F} \_$N: It is an RF I/O pin, a negative terminal for RF I/P signal to LNA and $\mathrm{O} / \mathrm{P}$ signal from $\mathrm{PA}$.

- SI: It is a digital input for serial configuration interface. 


\subsubsection{Application Circuit}

The application circuit of CC1101 transceiver is as shown in the Figure 6.2. The circuit shows various connections. To the left of the CC1101 we can see digital interface. The digital interface is the interface connected to the processor. The matching circuit is right of the CC1101, this combination of inductors and capacitors determine the frequency of operation. The values of the components are as shown in the Table 6.1 .

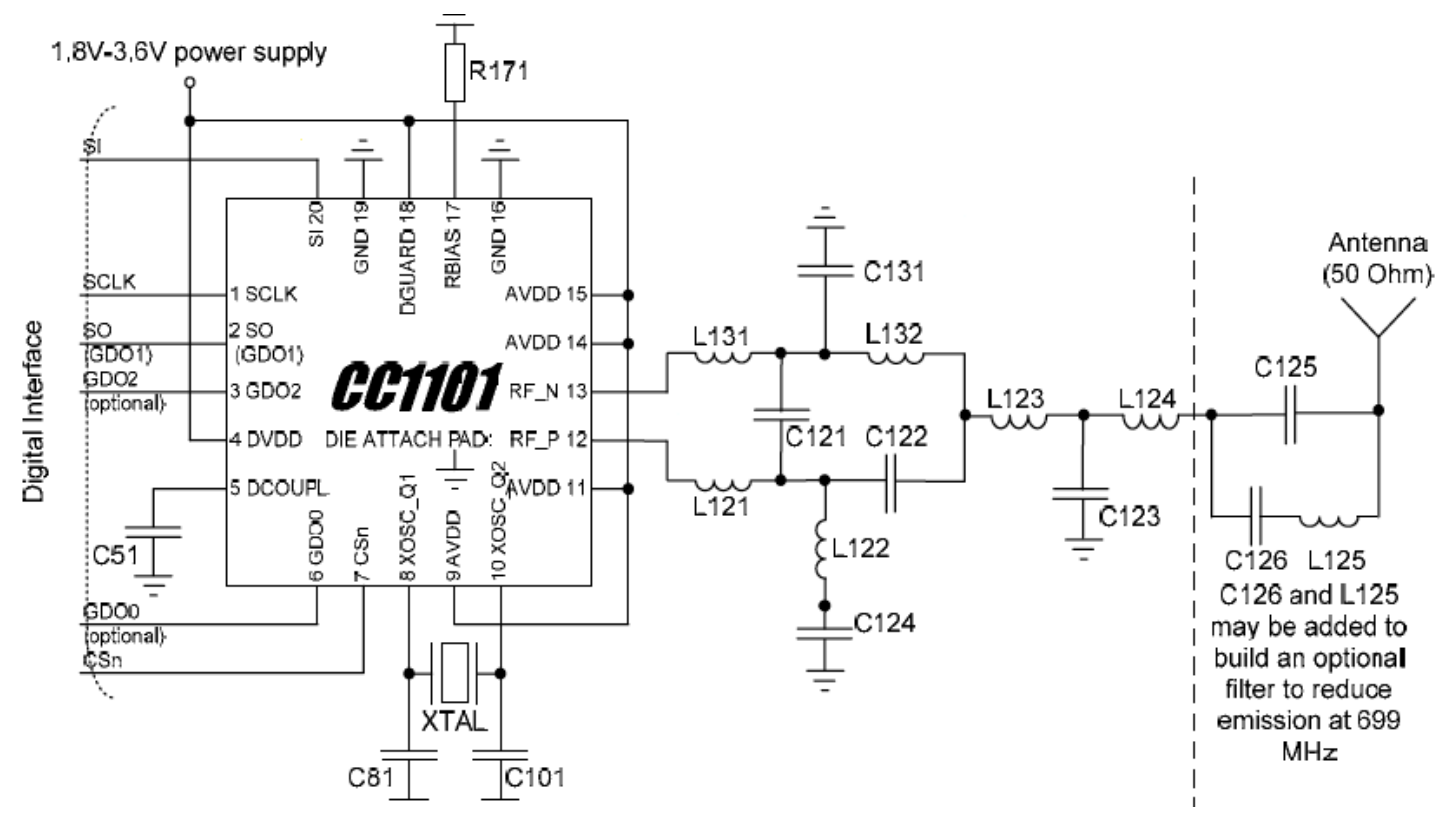

Figure 6.2. A typical application circuit for $915 \mathrm{MHz}[1]$. 
Table 6.1. Bill of material for the application circuit [1]

\begin{tabular}{|c|c|c|}
\hline Components & Value & Manufacturer \\
\hline $\mathrm{C} 15$ & $\begin{array}{l}\text { 100nF } \pm 10 \%, 0402 \\
\text { X5R }\end{array}$ & Murata GRM1555C series \\
\hline $\mathrm{C} 81$ & $\begin{array}{l}27 \mathrm{pF} \pm 5 \%, \\
\mathrm{NPO}\end{array}$ & Murata GRM1555C series \\
\hline C101 & $\begin{array}{l}27 \mathrm{pF} \\
\mathrm{NPO}\end{array}$ & Murata GRM1555C series \\
\hline C121 & $\begin{array}{l}1.0 \mathrm{pF} \pm 0.25 \mathrm{pF} \\
0402 \mathrm{NPO}\end{array}$ & Murata GRM1555C series \\
\hline $\mathrm{C} 122$ & $\begin{array}{l}1.5 \mathrm{pF} \quad \pm \quad 0.25 \mathrm{pF} \\
0402 \mathrm{NPO}\end{array}$ & Murata GRM1555C series \\
\hline C123 & $\begin{array}{l}3.3 \mathrm{pF} \quad \pm \quad 0.25 \mathrm{pF} \\
0402 \mathrm{NPO}\end{array}$ & Murata GRM1555C series \\
\hline $\mathrm{C} 124$ & $\begin{array}{l}100 \mathrm{pF} \pm 5 \%, 0402 \\
\text { NPO }\end{array}$ & Murata GRM1555C series \\
\hline $\mathrm{C} 125$ & $\begin{array}{l}100 \mathrm{pF} \pm 5 \%, 0402 \\
\text { NPO }\end{array}$ & Murata GRM1555C series \\
\hline $\mathrm{C} 126$ & $\begin{array}{l}2.2 \mathrm{pF} \pm 0.25 \%, 0402 \\
\mathrm{NPO}\end{array}$ & Murata GRM1555C series \\
\hline $\mathrm{C} 127$ & $\begin{array}{l}2.2 \mathrm{pF} \pm 0.25 \%, 0402 \\
\mathrm{NPO}\end{array}$ & Murata GRM1555C series \\
\hline $\mathrm{C} 131$ & $\begin{array}{lrl}1.5 \mathrm{pF} & \pm & 0.25 \mathrm{pF} \\
0402 \mathrm{NPO} & \end{array}$ & Murata GRM1555C series \\
\hline L121 & $\begin{array}{l}\text { 12nH } \pm 5 \%, 0402 \\
\text { monolithic }\end{array}$ & Murata LQG15HS series \\
\hline L122 & $\begin{array}{l}18 \mathrm{nH} \pm 5 \%, 0402 \\
\text { monolithic }\end{array}$ & Murata LQG15HS series \\
\hline L123 & $\begin{array}{l}12 \mathrm{nH} \pm 5 \%, 0402 \\
\text { monolithic }\end{array}$ & Murata LQG15HS series \\
\hline L124 & $\begin{array}{l}12 \mathrm{nH} \pm 5 \%, 0402 \\
\text { monolithic }\end{array}$ & Murata LQG15HS series \\
\hline L125 & $\begin{array}{l}9.1 \mathrm{nH} \pm 5 \%, 0402 \\
\text { monolithic }\end{array}$ & Murata LQG15HS series \\
\hline L131 & $\begin{array}{l}12 \mathrm{nH} \pm 5 \%, 0402 \\
\text { monolithic }\end{array}$ & Murata LQG15HS series \\
\hline L132 & $\begin{array}{l}18 \mathrm{nH} \pm 5 \%, 0402 \\
\text { monolithic }\end{array}$ & Murata LQG15HS series \\
\hline $\mathrm{R} 171$ & $56 \mathrm{k} \Omega \pm 1 \%, 0402$ & Koa RK73 series \\
\hline XTAL & $26.0 \mathrm{MHz}$ & NDk, AT-41CD2 \\
\hline
\end{tabular}




\subsubsection{Radio Control}

The transceiver is having a built-in state machine that can be used in switching between different modes. It clearly shows how different command strobes can be used to change the states. It is important for the thesis as the Blocking Reader need to know the operational states. The status register MARCSTATE contains the current state number, the number are as shown in the state diagram. To design a Reader it is important to know when to switch between in the transmitter mode to receiver mode. In case of a passive tag communication power plays important role. So the switching time should be as small as possible. The radio control state diagram is as shown in the Figure 6.3.

\subsubsection{Packet Handling Hardware Support}

CC1101 transceiver is having built-in hardware support for packet oriented radio protocols. The Gen2 protocol is a packet oriented protocol so it makes it easy to program the CC1101. The packet handler can be configured to add preamble, sync word and the CRC. And also programmed not to send any of these. The thesis uses packet handling but configures the transmitter not to send any preamble, sync or CRC. As the blocking reader uses the same Gen2 Reader protocol the preamble or frame sync is different from that the packet handling of CC1101. Later in this chapter we will discuss the configuration of different registers and see how the Gen2 packet is sent. The packet format is as shown in the Figure 6.4. 


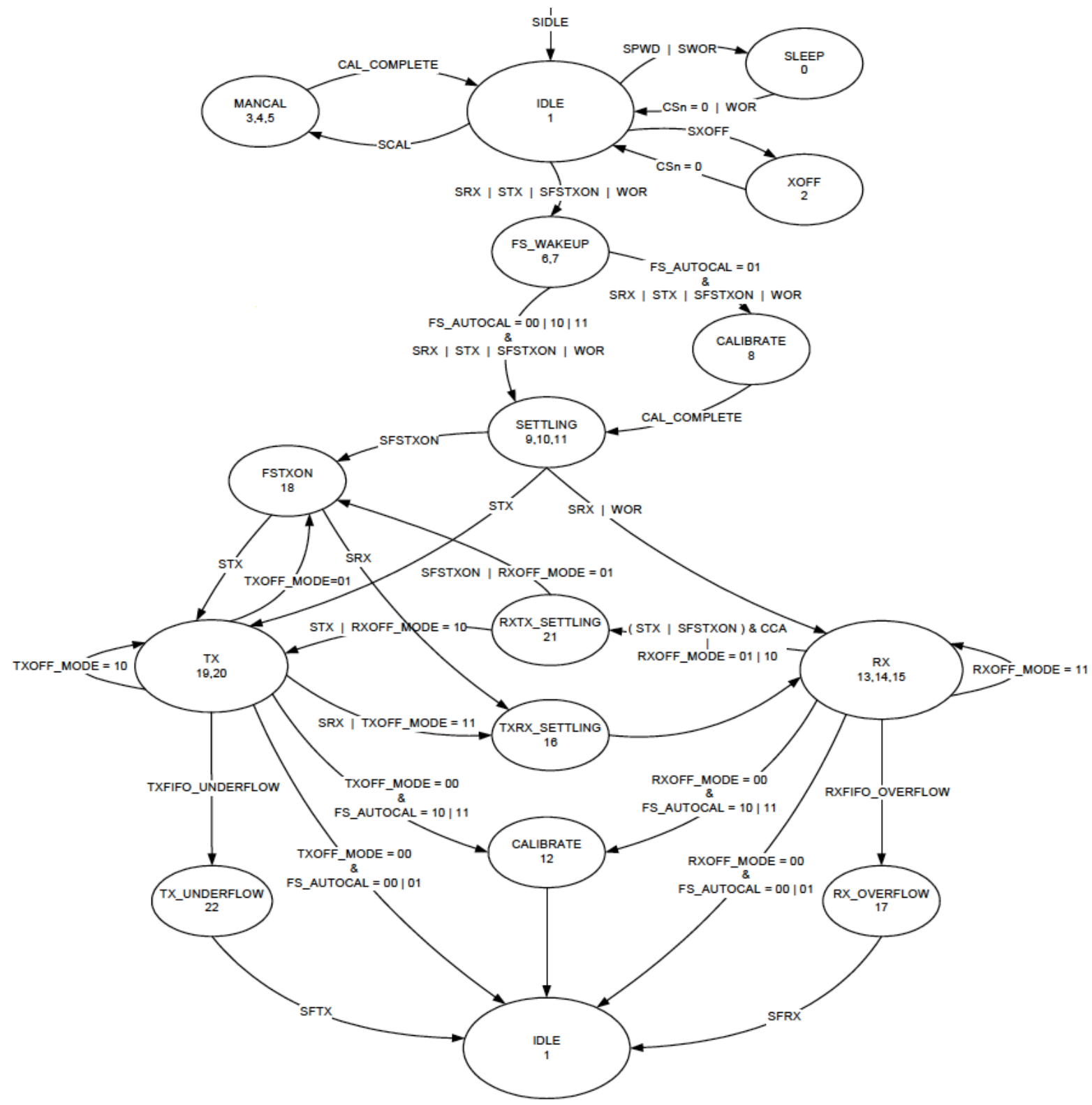

Figure 6.3. Complete radio control state diagram [1].

\subsubsection{Programming of CC1101}

The transceiver can be programmed by connecting to the micro-controller. The transceiver can be programmed to different modes. The buffer data of the transceiver 


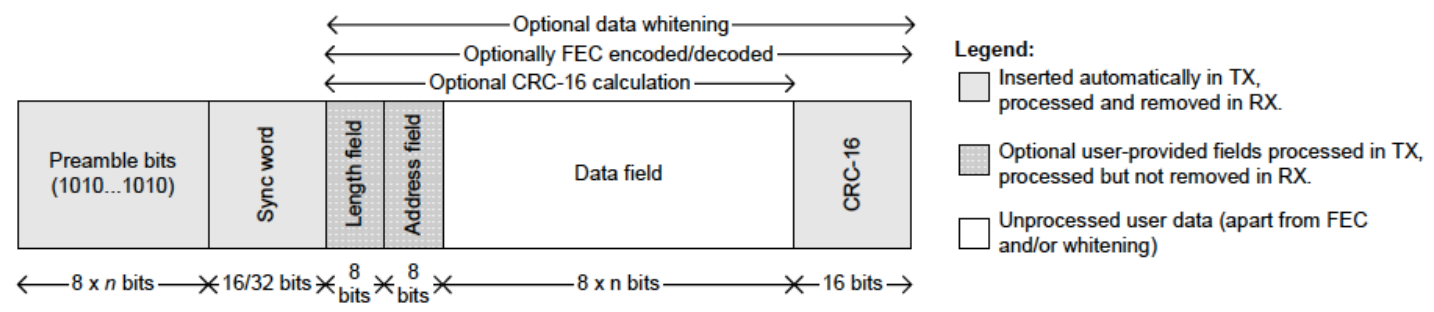

Figure 6.4. Packet format of CC1101 [1] .

can be read or written to the buffer memory. The 4 wire SPI-bus configuration can be used to read the status information.

\subsubsection{Amplifier}

The amplifier used in this design is RF5110 a 3V GSM power amplifier. This is a high efficiency power amplifier Some important features are

- Operates at frequency ranges from $800 \mathrm{MHz}$ to $900 \mathrm{MHz}$.

- Controllable output power with maximum power output of $+36 \mathrm{dBm}$.

- Needs a signal 2.7 to 4.8 supply voltage.

- Optimum efficiency of about $57 \%$.

The application circuit diagram is as shown in the Figure 6.5 


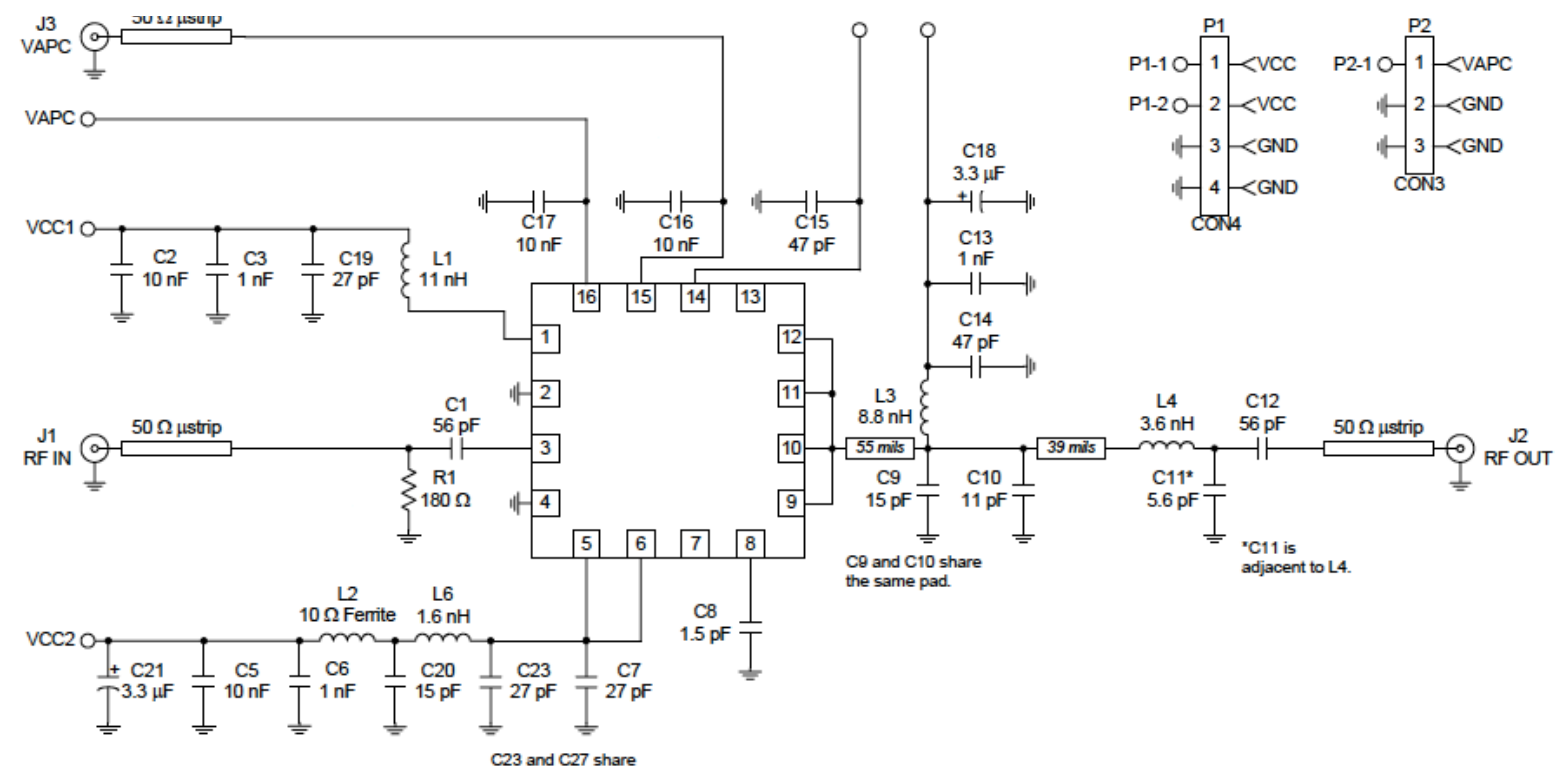

Figure 6.5. Application circuit diagram of RF5110 [2] .

\subsection{CC1101 Register Settings}

\subsubsection{Data Rate}

MDMCFG3 is a 8 bit register $D R A T E \_M[7: 0]$, which contains the mantissa of the data rate and MDMCFG4[DRATE_E[3:0]] is the exponent defined by the Equation 6.1.

$$
R_{D A T A}=\frac{\left(256+D R A T E \_M\right) \cdot 2^{D A T A_{-} E}}{2^{28}} \cdot f_{X O S C}
$$

The data rate is configured for $80 \mathrm{kBaud}$. The value chosen for MDMCGG3 is 0x93(h) and DRATE_E[3:0] is ' $1011^{\prime}(\mathrm{d})$.

\subsubsection{Frequency}

The frequency can be configured by setting 3 frequency control words FREQ0, FREQ1 and FREQ2 which are 8 bit length each. It is defined by the Equation 6.2. 
The center frequency is selected to be $915 \mathrm{MHz}$ for this the value of the registers are 0x3B, 0x31 and 0x23 respectively.

$$
f_{\text {carrier }}=\frac{f_{X O S C}}{2^{16}} \cdot F R E Q[23: 0]
$$

\subsubsection{Bandwidth}

The channel bandwidth can be set by the MDMCFG4 register which contains $C H A N B W_{-} E[1: 0]$ and $C H A N B W_{-} M[1: 0]$ as specified in the Equation 6.3. A default value of $203 \mathrm{kHz}$ is chosen, value being $0 \mathrm{x} 2 \mathrm{D}$.

$$
B W_{\text {channel }}=\frac{f_{X O S C}}{8 \cdot\left(4+C H A N B W_{-} M\right) \cdot 2^{C H A N B W_{-} E}}
$$

\subsubsection{Modulation}

The Blocking Reader requires ASK modulation. It is selected by setting MOD_FORMAT[2:0] as 011 .

Other register settings of the transceiver are as shown in the table 6.2 
Table 6.2. Final register settings

\begin{tabular}{|c|c|c|}
\hline Register & Value & Desicription \\
\hline FSCTRL1 & $0 \mathrm{x} 0 \mathrm{C}$ & Frequency synthesizer control \\
\hline FSCTRL0 & $0 \mathrm{x} 00$ & Frequency synthesizer control \\
\hline FREQ2 & $0 \times 23$ & Frequency control word, high byte \\
\hline FREQ1 & $0 \times 31$ & Frequency control word, middle byte \\
\hline FREQ0 & $0 \times 3 B$ & Frequency control word, low byte \\
\hline MDMCFG4 & $0 \times 7 \mathrm{~B}$ & Modem configuration \\
\hline MDMCFG3 & $0 \times 93$ & Modem configuration \\
\hline MDMCFG2 & $0 \times 34$ & Modem configuration \\
\hline MDMCFG1 & $0 \times 22$ & Modem configuration \\
\hline MDMCFG0 & $0 \times \mathrm{xF} 8$ & Modem configuration \\
\hline CHANNR & $0 \mathrm{x} 00$ & Channel number \\
\hline DEVIATN & $0 \times 62$ & Modem deviation setting \\
\hline FREND1 & $0 \times \mathrm{xB} 6$ & Front end RX configuration \\
\hline FREND0 & $0 \times 11$ & Front end RX configuration \\
\hline MCSM0 & $0 \times 18$ & Main Radio Control State Machine configuration \\
\hline FOCCFG & $0 \times 1 D$ & Frequency Offset Compensation Configuration \\
\hline BSCFG & $0 \mathrm{x} 1 \mathrm{C}$ & Bit synchronization Configuration \\
\hline AGCCTRL2 & $0 \mathrm{x} 07$ & AGC control \\
\hline AGCCTRL1 & $0 \mathrm{x} 00$ & AGC control \\
\hline AGCCTRL0 & $0 \times 91$ & AGC control \\
\hline FSCAL3 & 0xEA & Frequency synthesizer calibration \\
\hline FSCAL2 & $0 \times 2 \mathrm{~A}$ & Frequency synthesizer calibration \\
\hline FSCAL1 & $0 \mathrm{x} 00$ & Frequency synthesizer calibration \\
\hline FSCAL0 & $0 \mathrm{x} 1 \mathrm{~F}$ & Frequency synthesizer calibration \\
\hline FSTEST & $0 \times 59$ & Frequency synthesizer calibration \\
\hline TEST2 & $0 \times 88$ & Various test settings \\
\hline TEST1 & $0 \times 31$ & Various test settings \\
\hline TEST0 & 0x09 & Various test settings \\
\hline FIFOTHR & $0 \mathrm{x} 07$ & RXFIFO and TXFIFO thresholds \\
\hline IOCFG2 & 0x29 & GDO2 output pin configuration \\
\hline IOCFG0D & $0 \mathrm{x} 06$ & GDO0 output pin configuration \\
\hline PKTCTRL1 & 0x04 & Packet automation control \\
\hline PKTCTRL0 & $0 \mathrm{x} 01$ & Packet automation control \\
\hline ADDR & $0 \mathrm{x} 00$ & Device address \\
\hline PKTLEN & $0 \mathrm{xFF}$ & Packet length \\
\hline
\end{tabular}




\subsection{Complete Circuit Diagram}

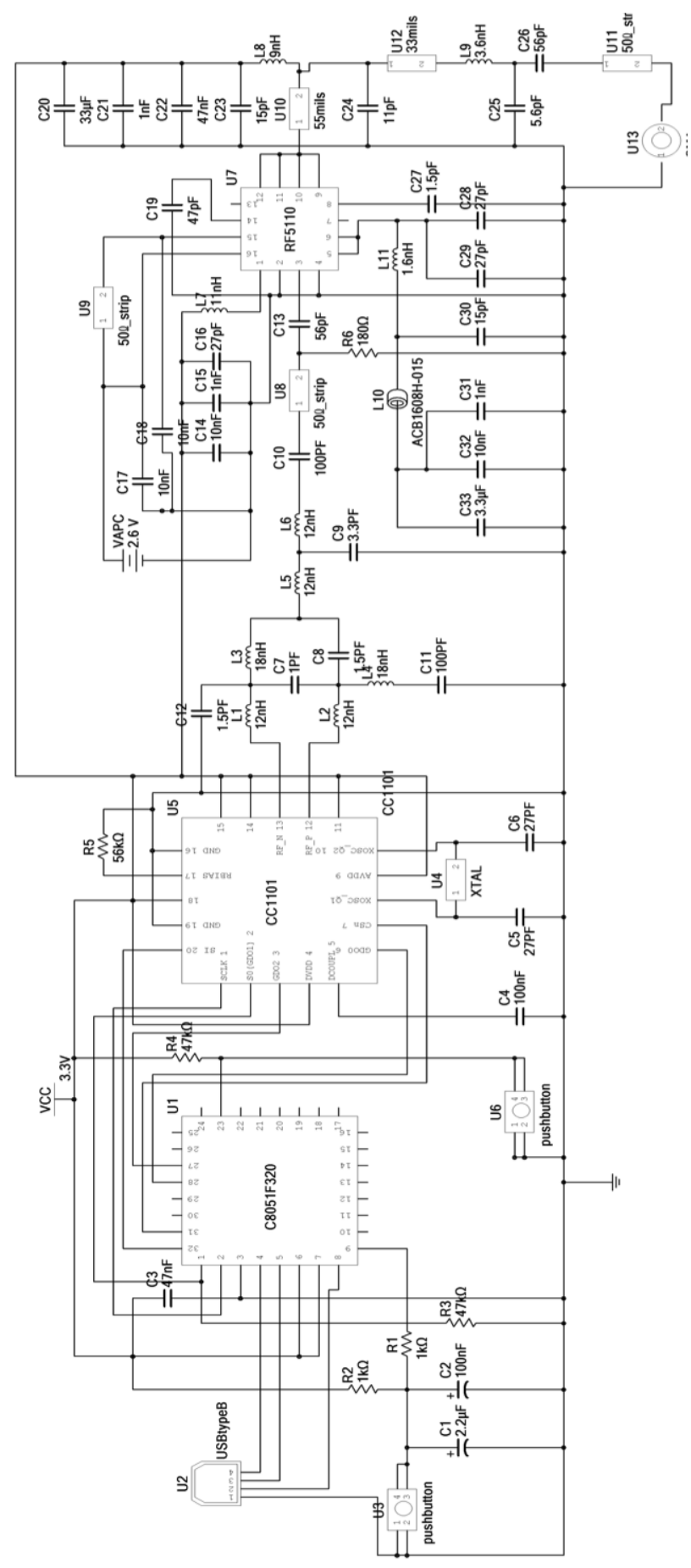

Figure 6.6. Complete circuit diagram of Blocking Reader. 


\subsection{Summary}

Using a low cost Reader design, Blocking Reader is developed. PIC C8015F320 provided a very good processing power with a $16 \mathrm{Kbyte}$ flash and $25 \mathrm{MHz}$ clock. The radio CC1101 is very powerful chip with easy to program packet handling and data rate. Flexible ASK shaping, suitable frequency hopping and programmable power up to $+10 \mathrm{dBm}$ provided with a optimum blocking range. Amplifier RF5110 is a low cost chip provided amplification of power improved the blocking range. The complete circuit diagram is as shown in Figure 6.6. Schematic designed in National Instruments Multisim 10.2. Complete Blocking Reader is estimated to be less than $\$ 30$. 


\section{CHAPTER 7}

\section{PERFORMANCE}

\subsection{Introduction}

This chapter contains different experiments and results to illustrate the performance of Blocking Reader. As the name specifies the Blocking Reader blocks the communication between Reader and Tag. Blocking Reader communicates with the Tag and Captures it. It uses the protocol against the Reader. A Sirit 501 Passive UHF RFID Reader is used in all the experiments. Alien Squiggle Gen2 Tag is used. And the Blocking reader with amplifier.

\subsection{Tag Response}

\subsubsection{Experimental setup}

In this experiment I would like to show the Tag response to the Query command of the Blocking Reader. The setup consist of a squiggle Tag, Blocking Reader and NI system used as sniffer. The NI system used in this experiment is National Instrument PXI-8106 embedded controller solely designed for LabView. Sniffer program displays the RF-915 MHz commands in time domain. In this experiment we basically use this NI system to show the Blocking Reader command and the Tag response.

\subsubsection{Tag Response Results}

The Figure 7.1 shows the RN 16 response of the Tag for the Query command of Blocking reader. Figure 7.2 shows the timings of $B R \Rightarrow$ Tag communication. Blocking Reader sends Query for every $1.4 \mathrm{~ms}$. Query commands are $0.8 \mathrm{~ms}$ of length later the 


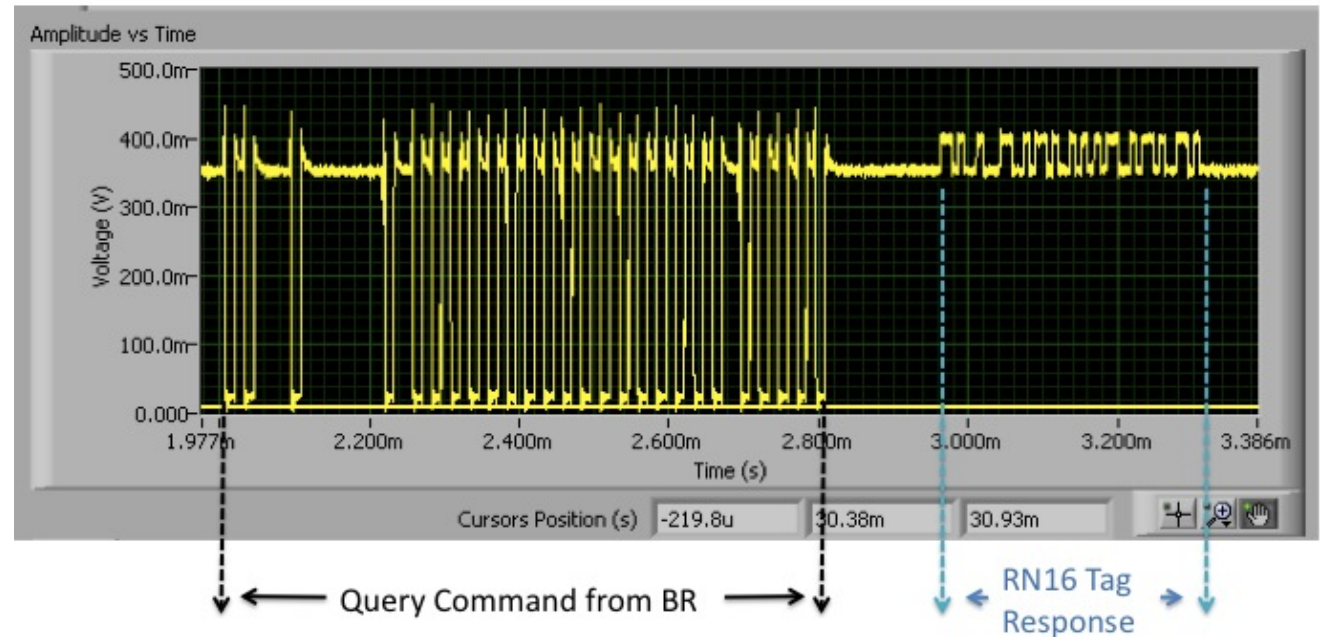

Figure 7.1. Tag Response as shown by Sniffer .

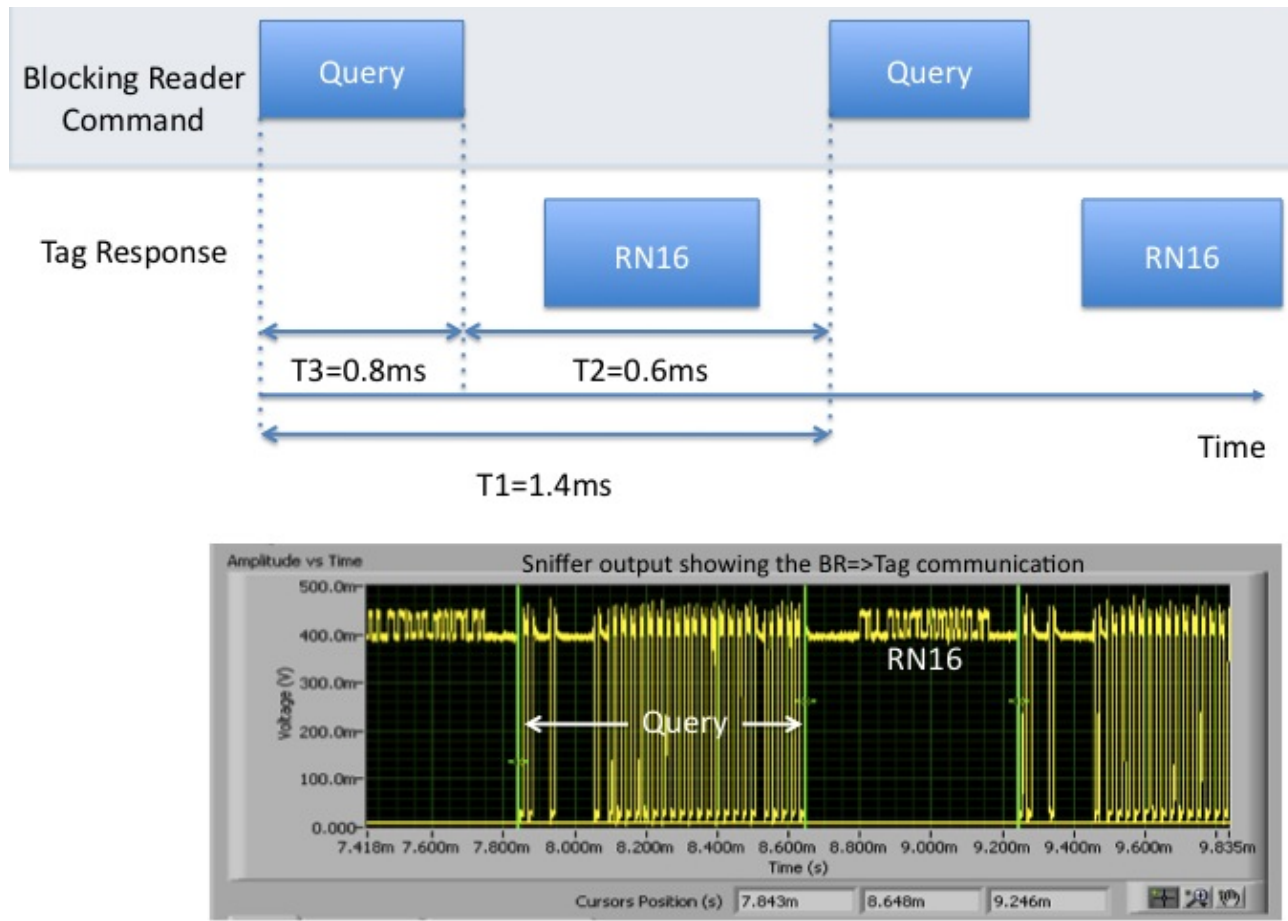

Figure 7.2. Tag Response as shown by Sniffer showing the timings. 
$\mathrm{BR}$ is programmed to send carrier wave for $0.6 \mathrm{~ms}$. In this time the Tag responds to the Query of BR.

\subsection{Maximum Tag Response Range}

\subsubsection{Experimental setup}

The setup is as shown in the Figure 7.3. The NI sniffer is placed next to the Tag and the BR is moved away from the Tag. The Tag response is seen in the sniffer and for every Tag response the value of voltage readings also noted. Here the BR power is set for $\mathrm{Vapc}=1.6 \mathrm{~V}$ of amplifier which gives $9-8 \mathrm{dBm}$.

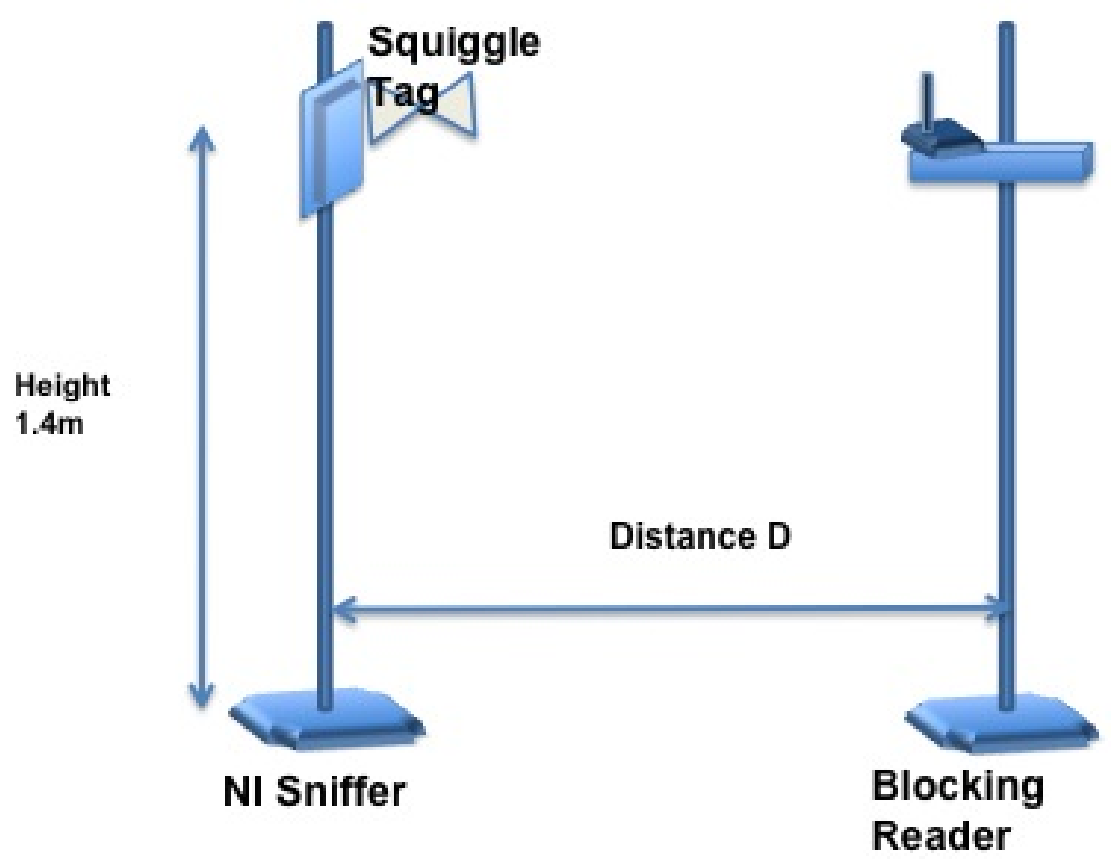

Figure 7.3. Experimental setup for Tag response range. 


\subsubsection{Results for Tag Response Range}

The Figure 7.4 shows Tag RN16 voltage with respect to distance from the Blocking Reader. As BR moved away from the Tag the voltage value of the Tag response is reducing. At $1.1 \mathrm{~m}$ the Tag stops responding to the BR query. There is an interesting pattern after $1.1 \mathrm{~m}$. It is too complex to say the reason for this pattern. We can say it might be due to multi-path, the places where Tag is not responding, it might have destructive interference. Finally we conclude that the maximum Tag response range is $1.1 \mathrm{~m}$ for a $+6-9 \mathrm{dBm}$ power of $\mathrm{BR}$.

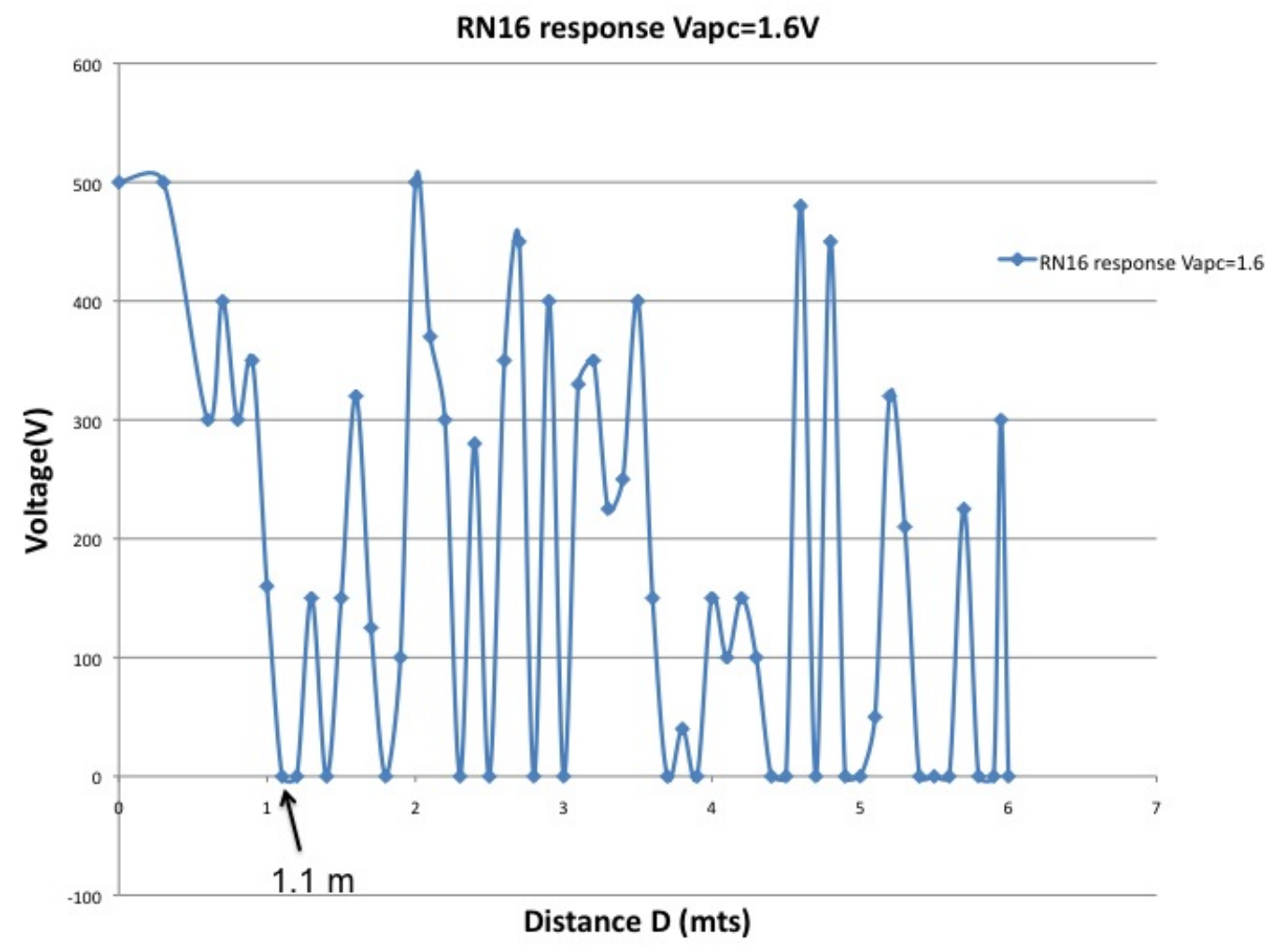

Figure 7.4. Tag response range. 


\subsection{Illustration of Blocking}

\subsubsection{Experimental setup 1}

In this experiment a sirit Reader and Tag are placed at $0.9 \mathrm{~m}$ apart. And a Blocking Reader is placed at a distance of $\mathrm{D}$ from the Tag as shown in the Figure 7.5. In this setup a optimum distance is selected between the Sirit Reader and the Tag where there is very good read rate. The Blocking reader is brought closer to the Tag and the Distance from the tag to the Blocking Reader and Tag is plotted verses the read rate as seen in the Sirit Reader.

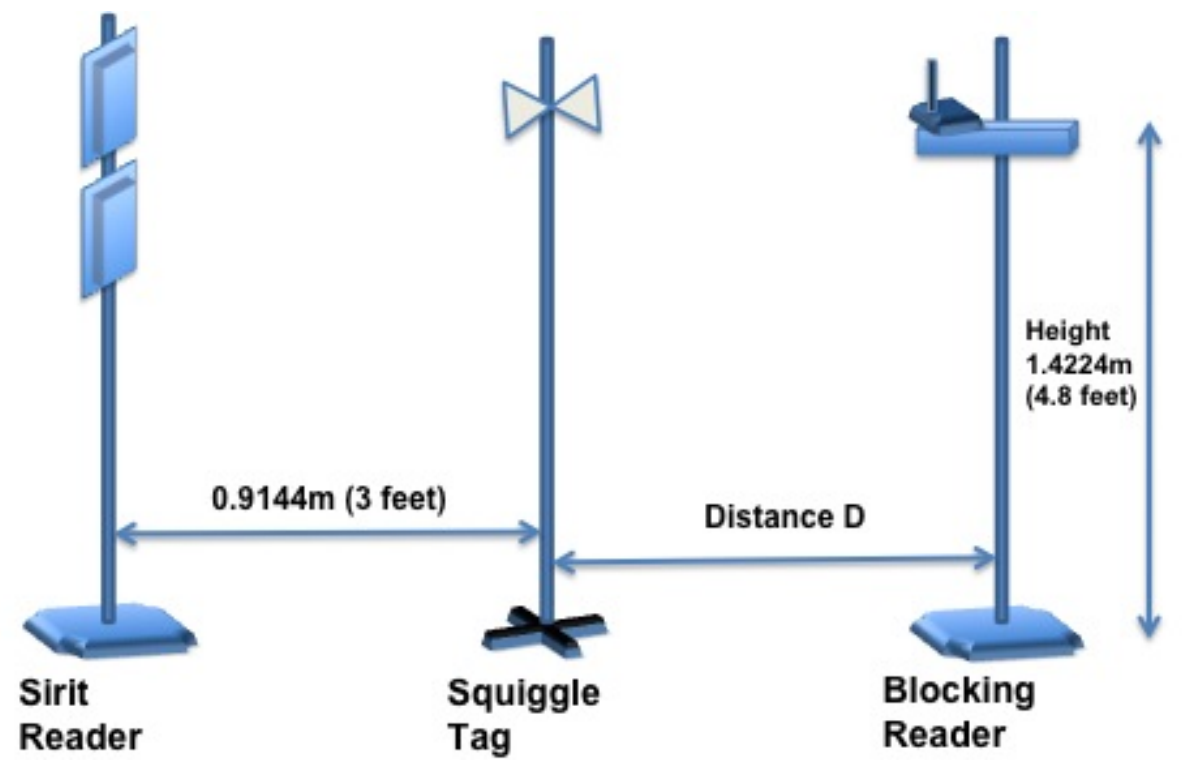

Figure 7.5. Experimental setup1 of Blocking Reader illustration .

\subsubsection{Results for experimental setup 1}

The Results are as shown in the Figure 7.6 is for experimental setup shown in the Figure 7.5. Here we can see that when the blocking reader is moved away from the Tag the Read Rate readings of the Sirit Reader are taken. There are absolutely 
no read until there is Blocking Reader is moved to a distance of $1.3 \mathrm{~m}$ There are two different readings taken. One with the Vapc value of the Amplifier $1.6 \mathrm{~V}$ which can give a maximum power of $+6.8 \mathrm{dBm}$ and Vapc value $2.6 \mathrm{~V}$ which can give a maximum power of $+21 \mathrm{dBm}$. For $\mathrm{Vapc}=1.6 \mathrm{~V}$ there is a maximum blocking range of about $1.3 \mathrm{~m}$ and for $\mathrm{Vapc}=2.6 \mathrm{~V}$ the maximum blocking range of $1.35 \mathrm{~m}$.

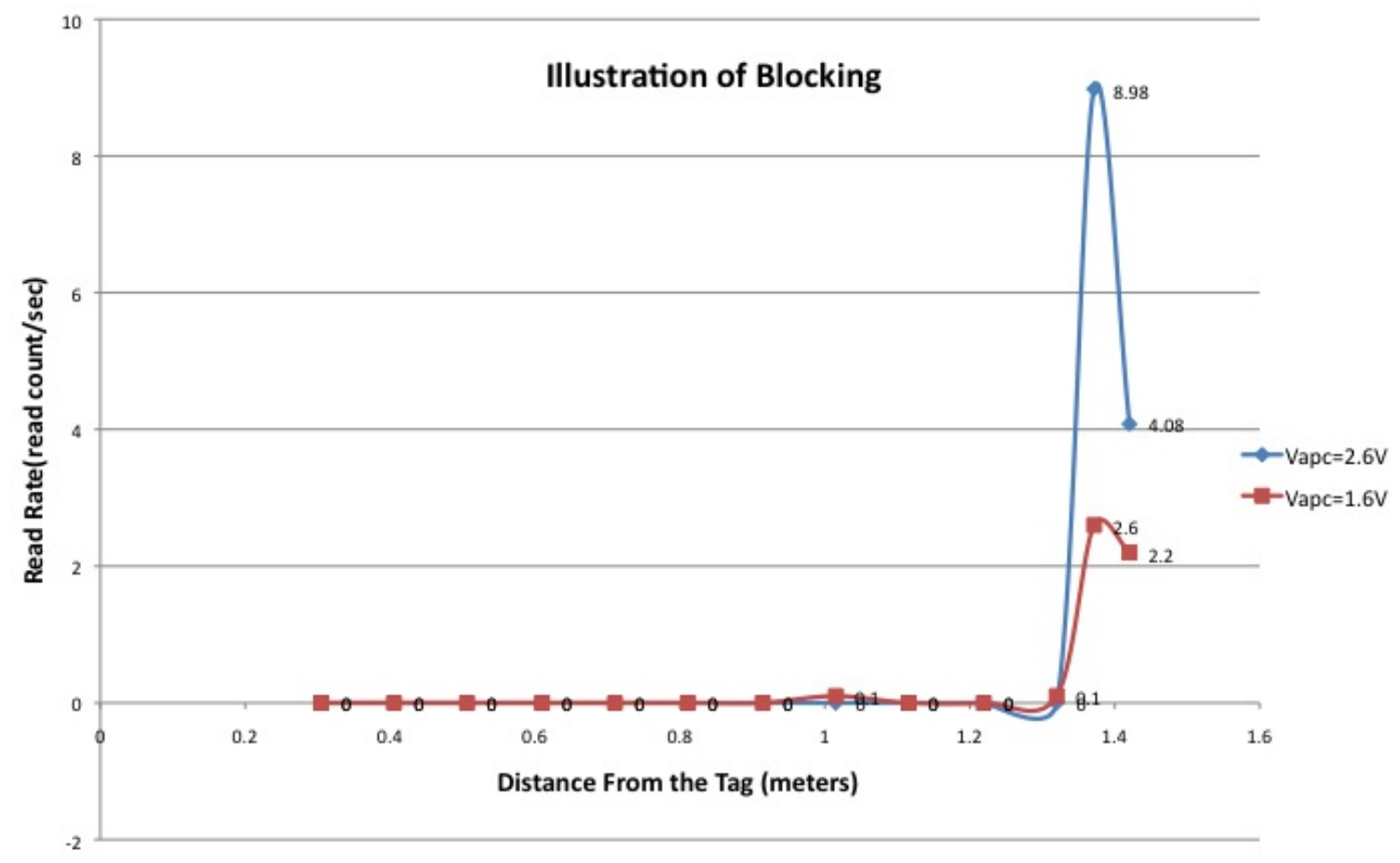

Figure 7.6. Graph of distance (D) vs read rate for experimental setup1.

Figure 7.7 shows that read rate calculated with the same setup distance varying till $5.4 \mathrm{~m}$. In this experiment we could see some interesting patterns. The blocking reader is able to block in some distances after that $1.3 \mathrm{~m}$. But the some times its not. In this Figure 7.7 the read rate is measured for distances from the tag varying from $0.3 \mathrm{~m}$ to $5.4 \mathrm{~m}$. The graphs are drawn for two Vapc values $1.6 \mathrm{~V}$ which gives about 
$9.1 \mathrm{dBm}$ power and $2.6 \mathrm{~V}$ which gives about $21 \mathrm{dBm}$ of power. Also there is a sine wave plotted, just for explanation purpose. Here we can clearly see the pattern of reads are following the same patten as sine wave. Where there is a positive peak of the sine wave we can notice that the blocking reader is actually blocking in some cases after $1.3 \mathrm{~m}$. As the blocking reader uses $915 \mathrm{MHz}$ frequency for communication. Power follows the pattern of sine wave for the communication. So this might be a possible reason. It should be noted that there is multi-path and reflections due to other objects around also causing some patterns. Assuming a reflected wave from floor if we plot a friss power adding two EM waves the power up-down follows the wave pattern of that frequency. It is obvious because of the phase, the adding can be constructive and destructive.

\subsubsection{Experimental setup 2}

In this setup the Sirit Reader and the Blocking Reader are kept at a constant distance of 1.5mapart and the Tag is moved away from the blocking reader as shown in the Figure 7.8. The negative values of the distances indicate that the tag is moved away from Blocking Reader and towards the Sirit Reader.

\subsubsection{Results for Experimental setup 2}

In this experimental setup 2 we can see that when the Tag is moved away from the Blocking Reader(BR) and Sirit Reader (SR) it can hardly be read except at one point. But when Tag is moved towards the SR away from BR then we can see that until certain point the Tag is not read. After the Tag passes $0.9 \mathrm{~m}$ mark the SR starts reading the Tag. We can explain this phenomenon by the free space power equation, Friss equation. 
Illustration of Blocking Reader

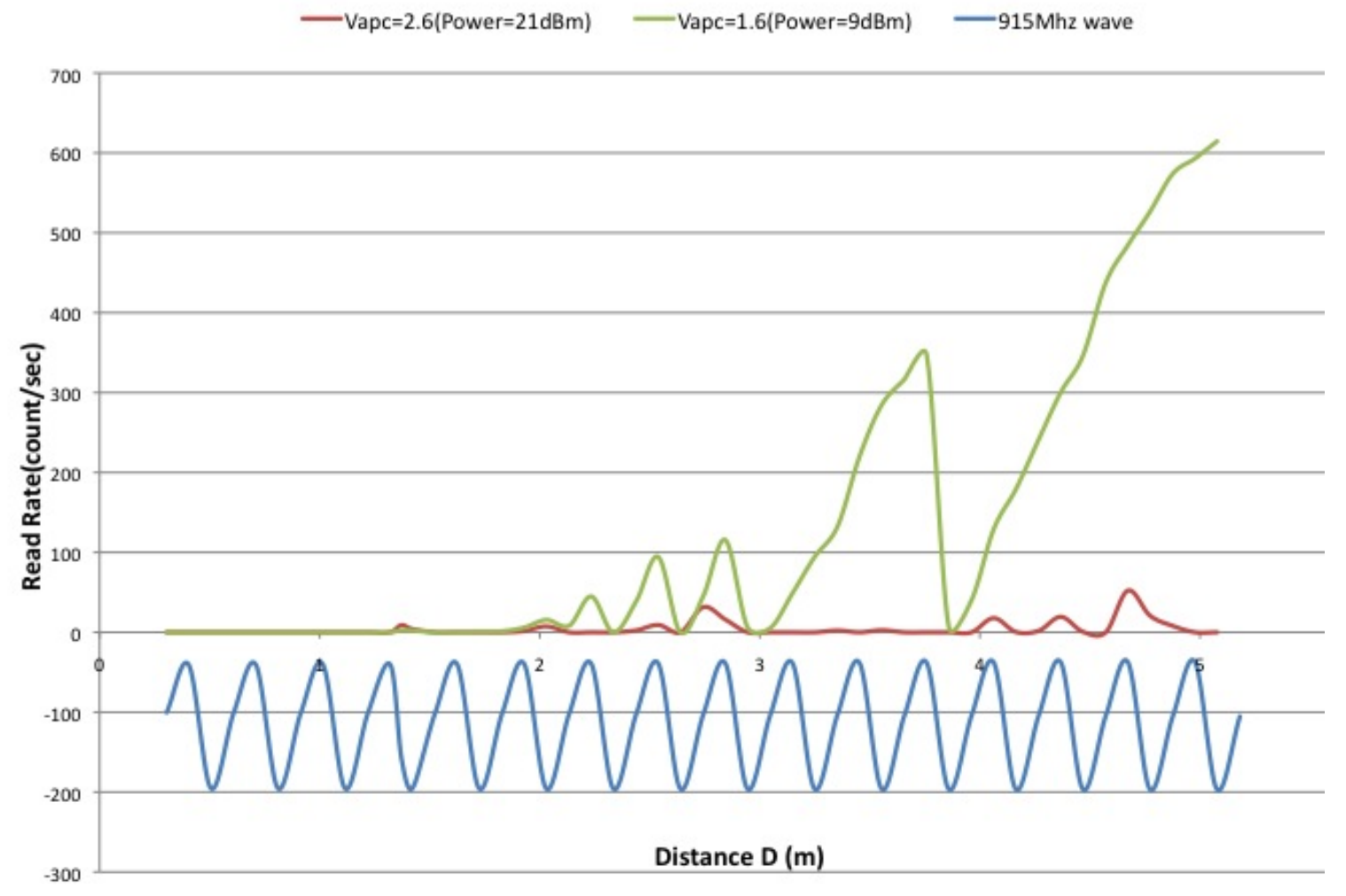

Figure 7.7. Graph of Distance (D) vs Read Rate.

Here there are two Readers, as we move away from the Reader power received at that point decreases. So When the Tag is moved away from the SR and BR the power received by the Tag from SR and BR decreases with distance. Here the Blocking Reader power is more than SR power as the BR is closer to SR. So it is very clear that the Tag should block any thing in this region.

Now when the Tag is moved between the SR and BR. Away from BR and towards SR the BR power received decreases and SR power received by the Tag increases hence at certain point the Tag starts to Read as show in the Figure 7.9. 


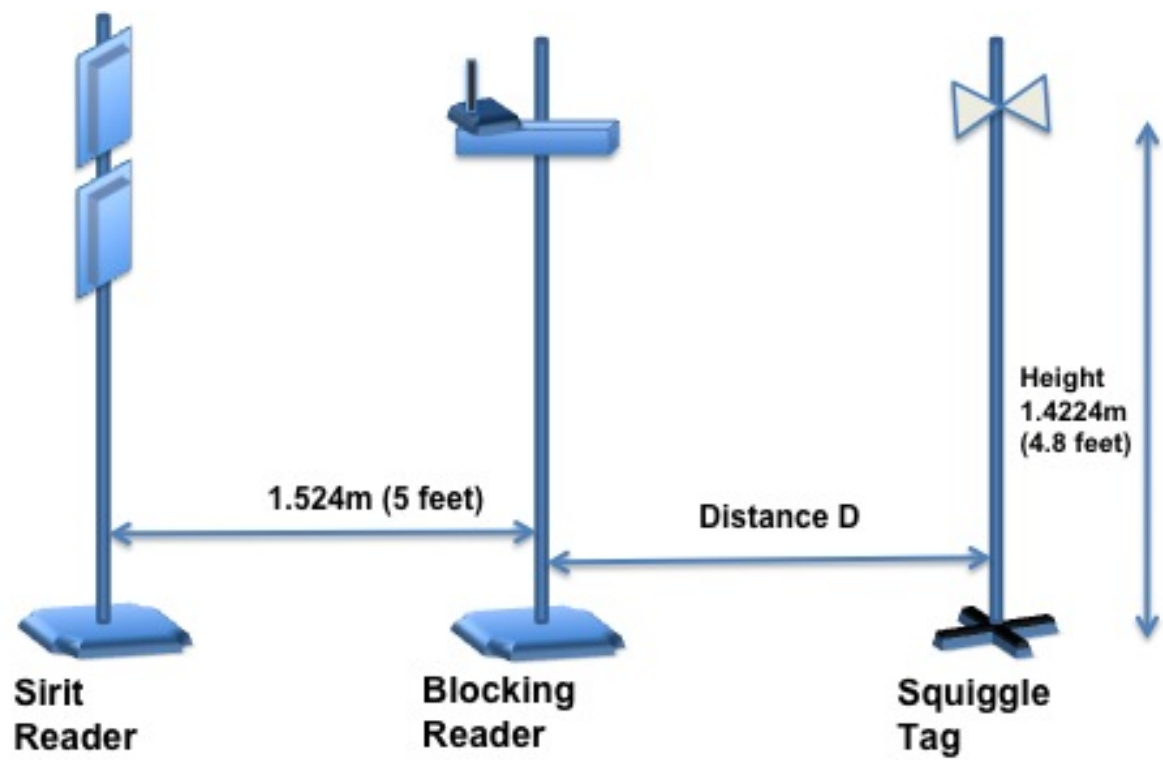

Figure 7.8. Experimental setup2 illustrating blocking.

In the Figure 7.10 we have plotted the Sirit Reader power and the Blocking Reader power with the help of the Friss free space power equation. This is the theoretical power value not considering any path losses or any multipath constructive and destructive interference. Here also we can see some interesting patterns. The Sirit reader states reading the Tag when the Tag is $0.9 \mathrm{~m}$ away from the Blocking Reader and then stops reading. Later in next $0.3 \mathrm{~m}$ that is $1.2 \mathrm{~m}$ it starts reading again and stop. This pattern repeats for $1.5 \mathrm{~m}$ but this time the sirit reader power is soo high that the blocking reader effects are overcome. 


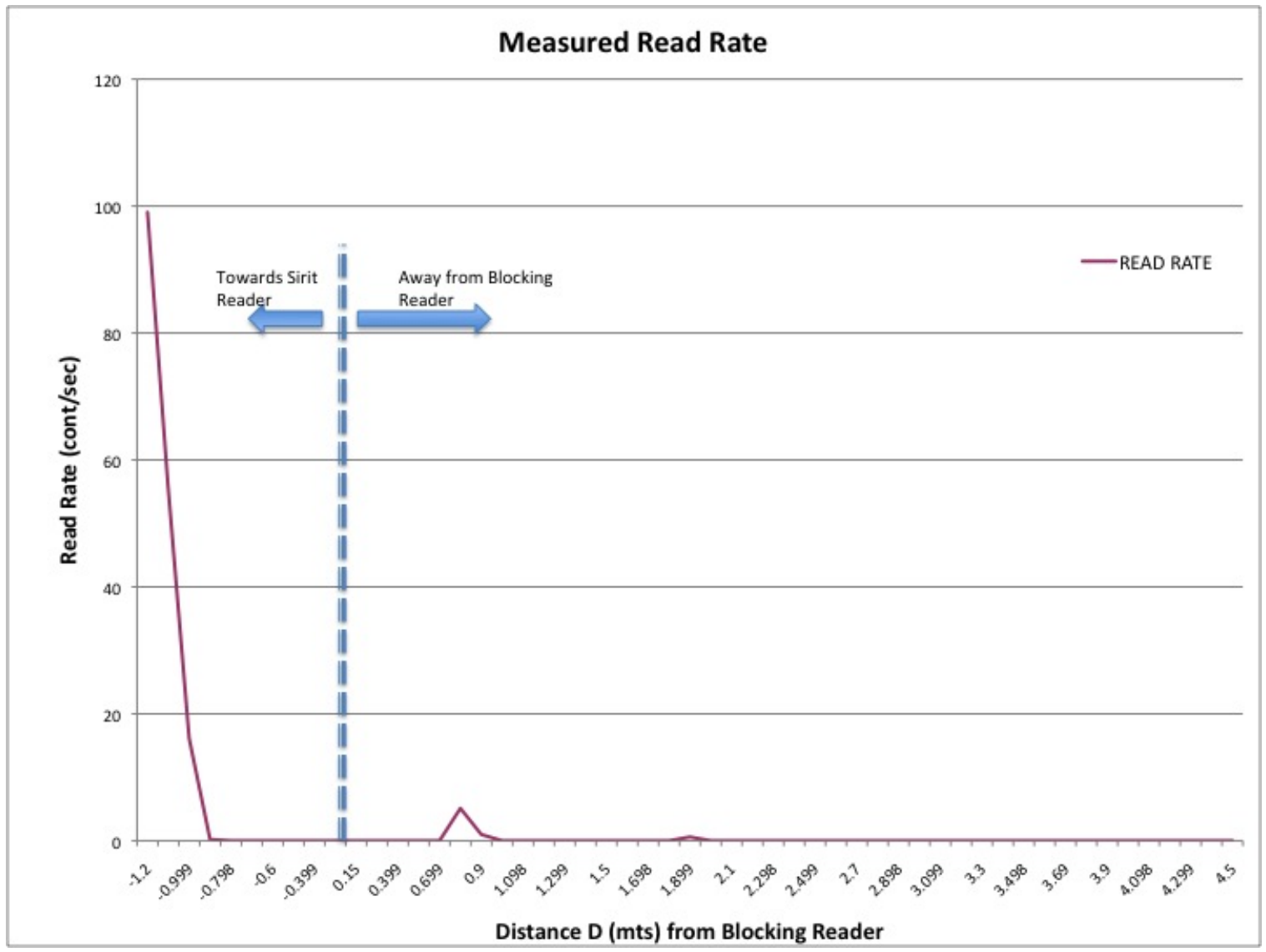

Figure 7.9. Graph of read rate with BR and SR fixed. 


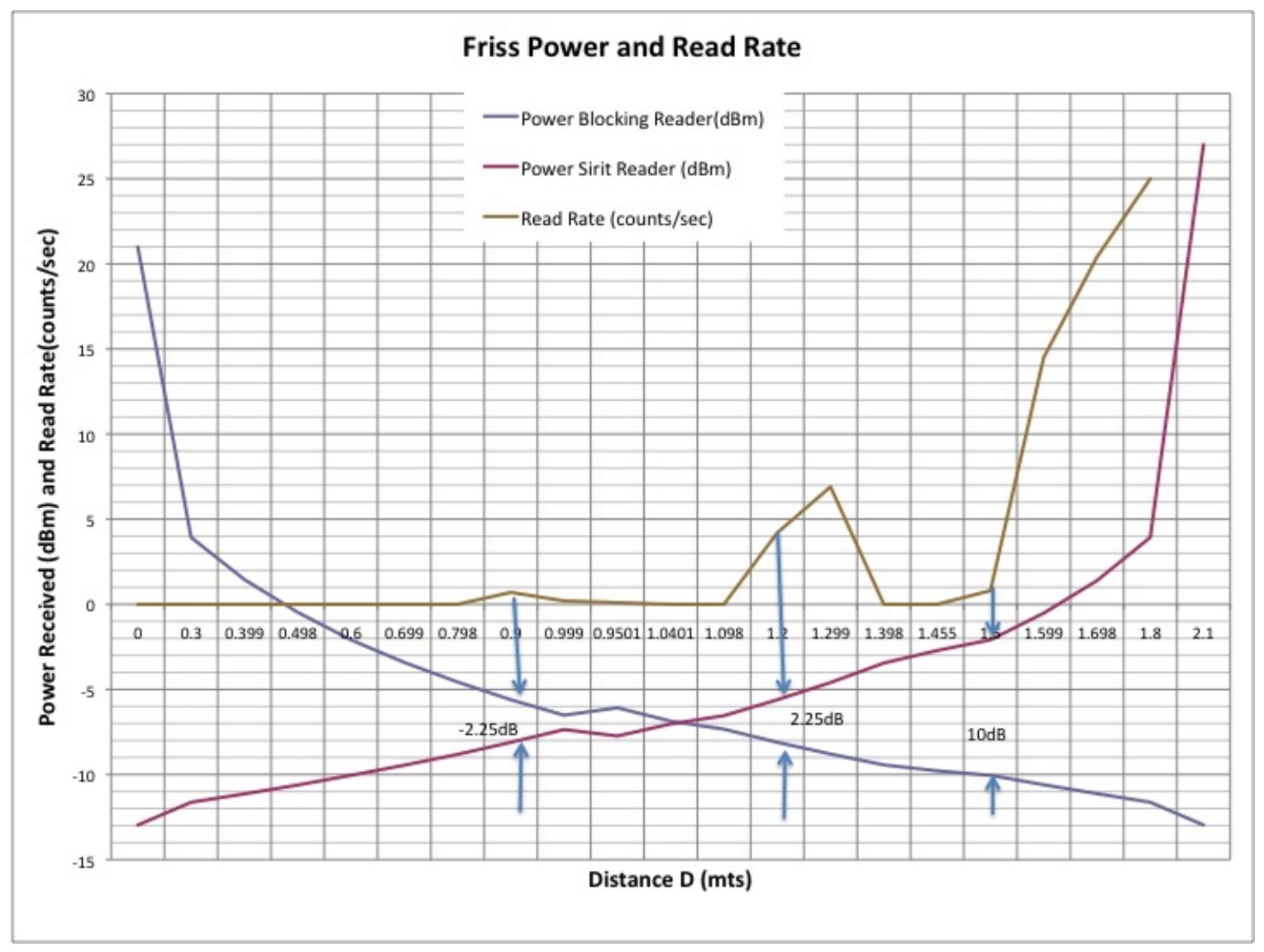

Figure 7.10. Comparing Friss Power for SR and BR with Read Rate. 


\subsection{Blocking Envelope}

\subsubsection{Blocking Envelope Around the Tag}

In this experimental setup shown in the Figure 7.11 the Tag and Sirit Reader are kept at a fixed position 2.1m apart and the Blocking Reader is moved around the Tag. Here only the first read is taken moving away from the Tag at every angle. This indicates the envelope around the Tag where the Blocking Reader is successfully blocking irrespective of any other settings.

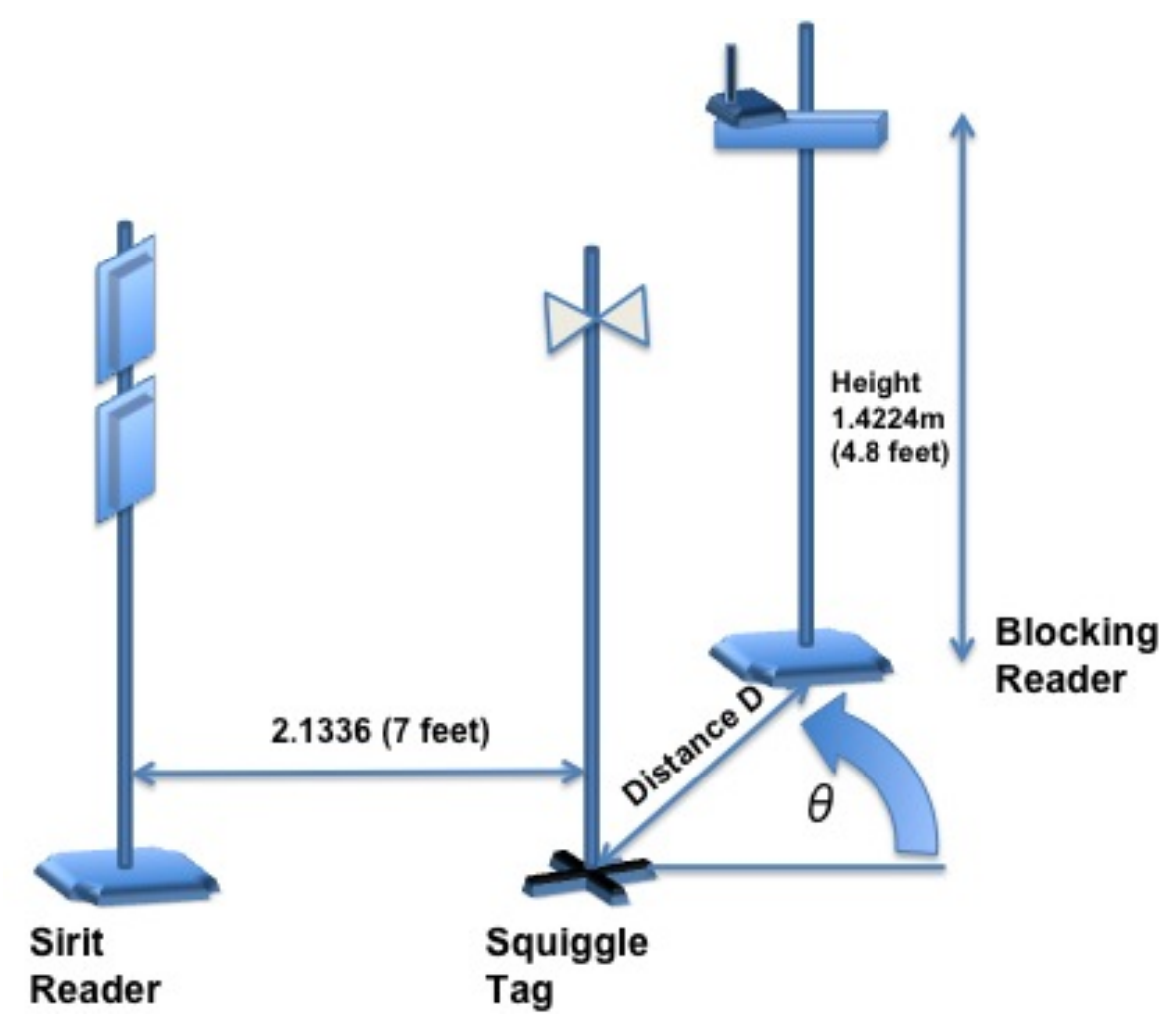

Figure 7.11. Setup illustrating blocking envelope around Tag. 


\subsubsection{Results}

The polar Figure 7.12 shows the maximum blocking distance the blocking reader should be present to successfully block a Tag. We can clearly see it is a envelope pattern matching the monopole radiation pattern. The average distance around the Tag was observed to about $0.9 \mathrm{~m}$.

\section{Maximum Blocking Envelope around Tag (mts)}

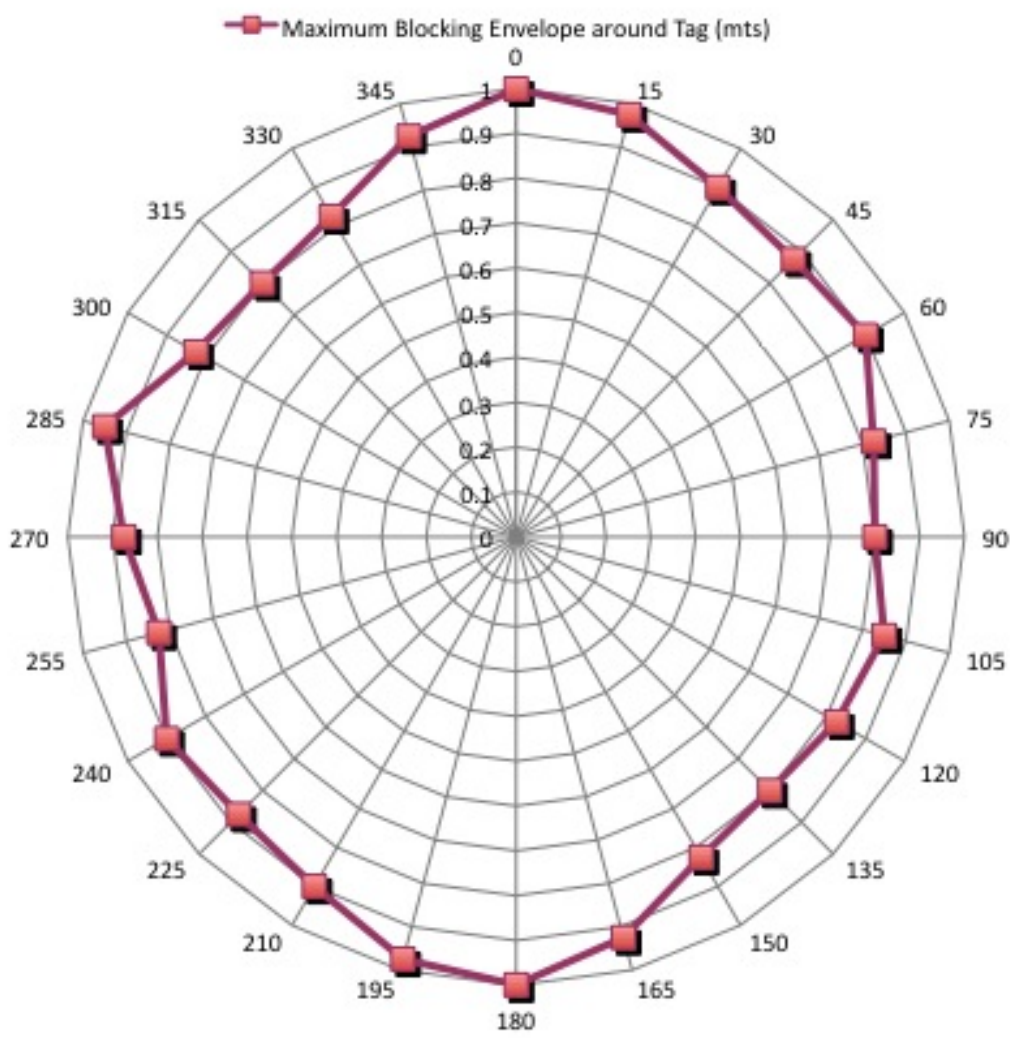

Figure 7.12. Blocking envelope around the Tag . 


\subsubsection{Blocking Envelope around the Blocking Reader}

In the setup show in the Figure 7.13 the Sirit Reader and Blocker Reader is fixed with distance between them 2.1m. The Tag is moved around the Blocker Reader to not the maximum blocking distance. The value of the Vapc is $2.6 \mathrm{~V}$ hence the Blocking Reader is radiating a power of about $+21 \mathrm{dBm}$. Here at each angle of 30 degree the Tag is moved closer and away from the Blocking Reader and a maximum distance where the Sirit Reader just starts to read is noted down.

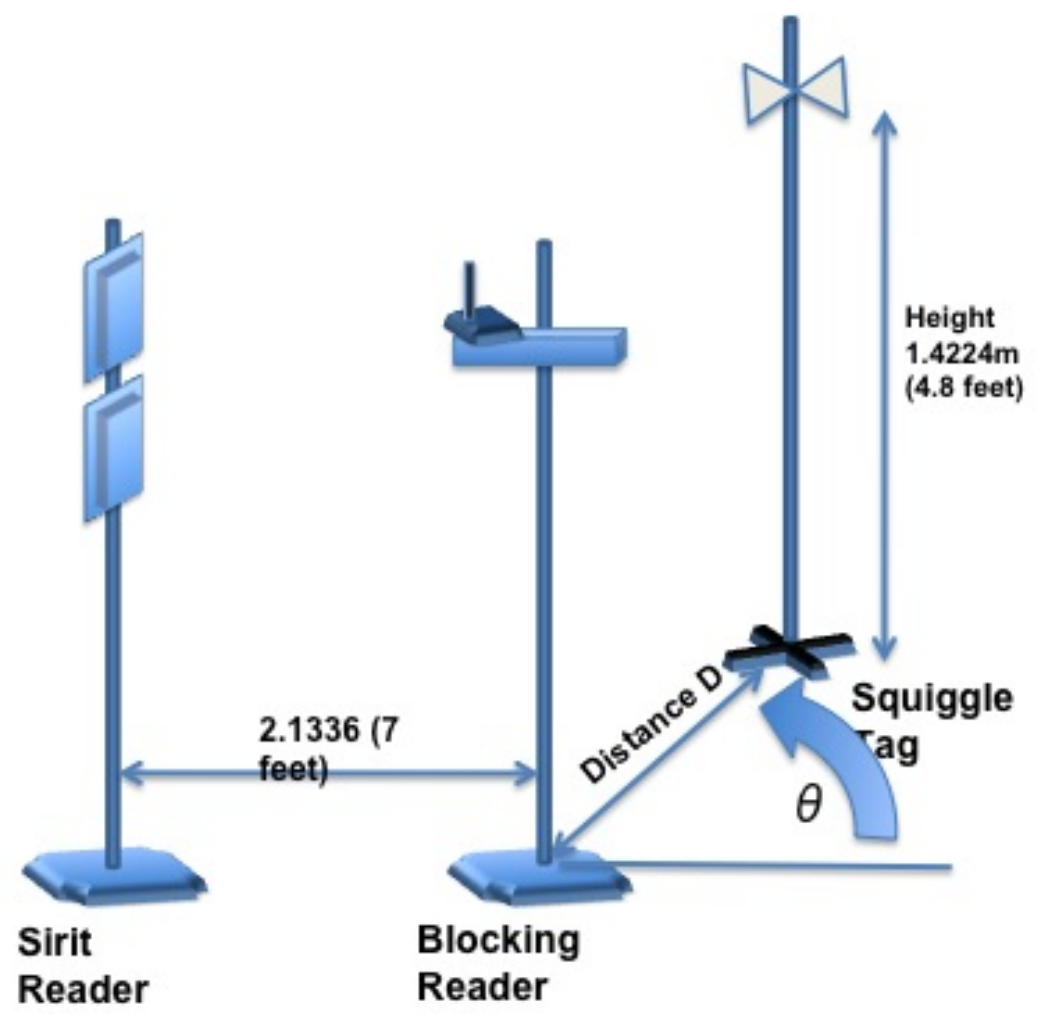

Figure 7.13. Setup illustrating blocking envelope around Blocking Reader. 


\subsubsection{Results}

The results of the experimental setup shown in the Figure 7.13 is plotted in the polar graph as shown in the Figure 7.14. Here we can see that the Blocking Reader has created an envelope around inside which the Tag is blocked from any communication from other readers.

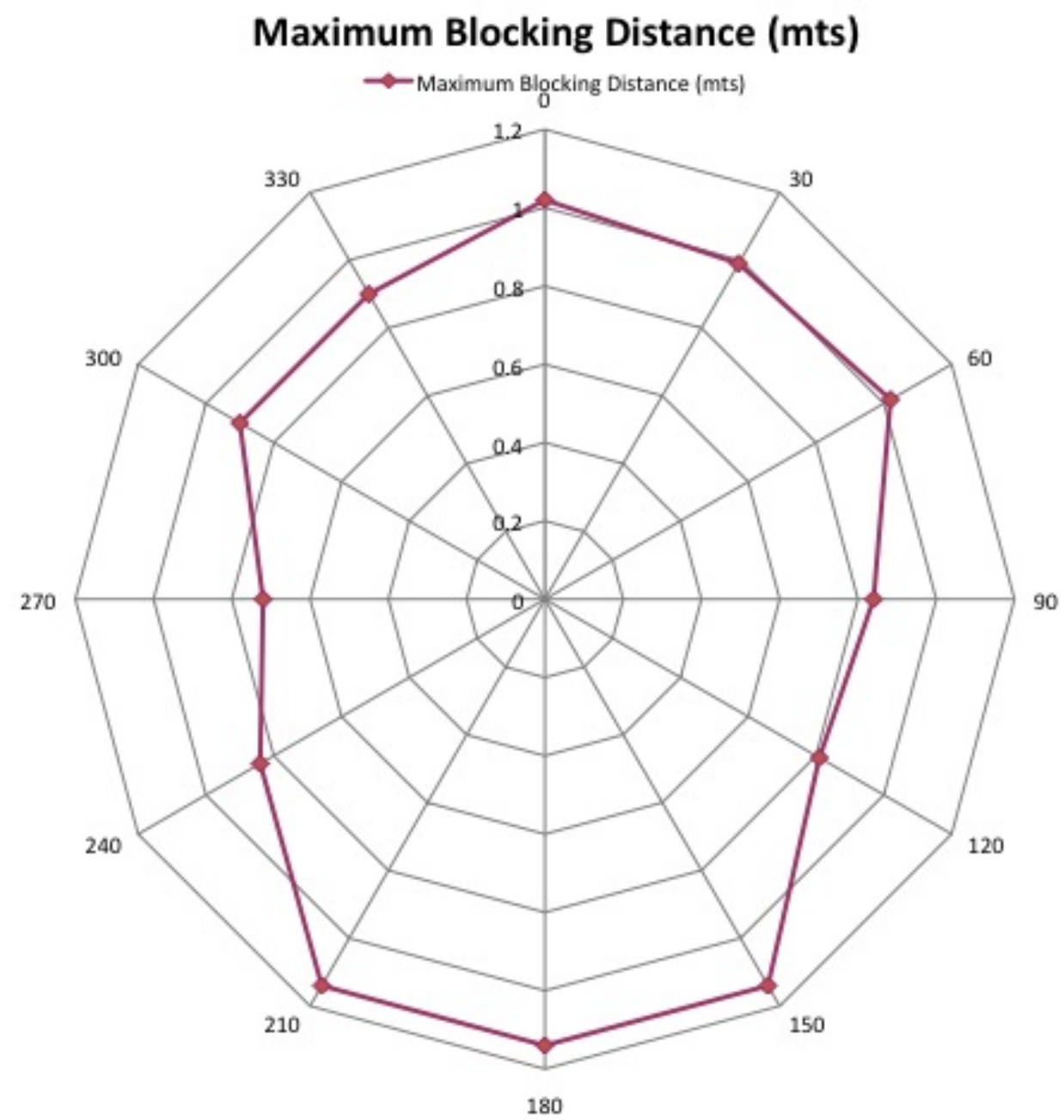

Figure 7.14. Blocking envelope around the Blocking Reader. 


\subsubsection{Blocking Envelope Vertical Axis}

The setup is as shown in the Figure 7.15, here the setup is same as the previous experiment instead of moving the horizontal axis the Tag is moved in the vertical axis. For every 45 degrees the distance between from the Blocking Reader is varied and the value of maximum blocking distance is noted.

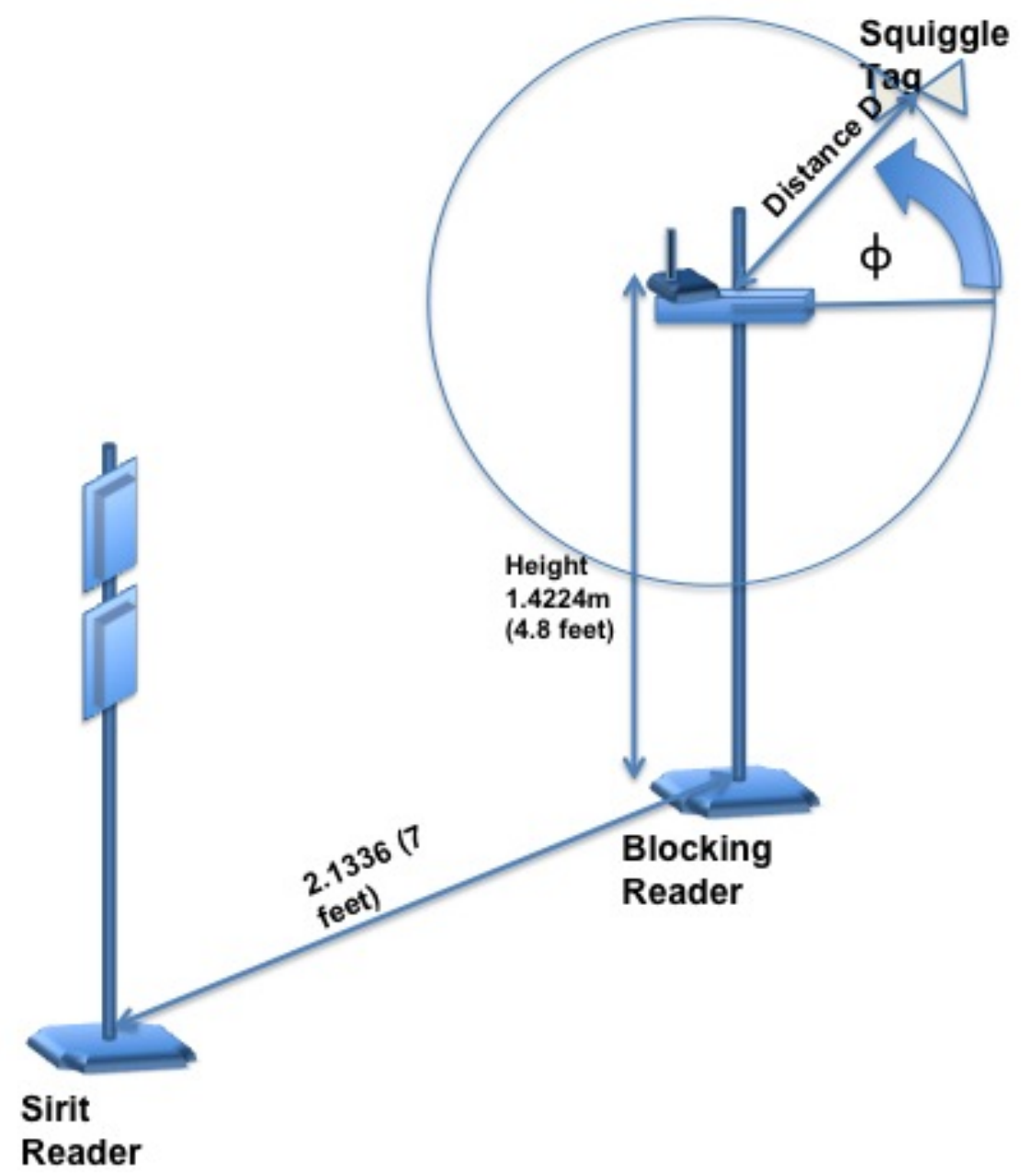

Figure 7.15. Setup illustrating blocking envelope in vertical axis. 


\subsubsection{Results}

The results of this setup is shown in the polar Figure 7.16. The pattern looks similar to the monopole radiation pattern in the $\mathrm{Z}$ axis. Obviously it should match with that pattern as the power of Blocking Reader at 0 and 180 degrees are low due to the nulls at that point the Tag is easily read by the Sirit Reader. We can see at 0 degrees the maximum blocking distance is just $0.01 \mathrm{~m}$.

\section{Maximum Blocking distance vertical axis (mts)}

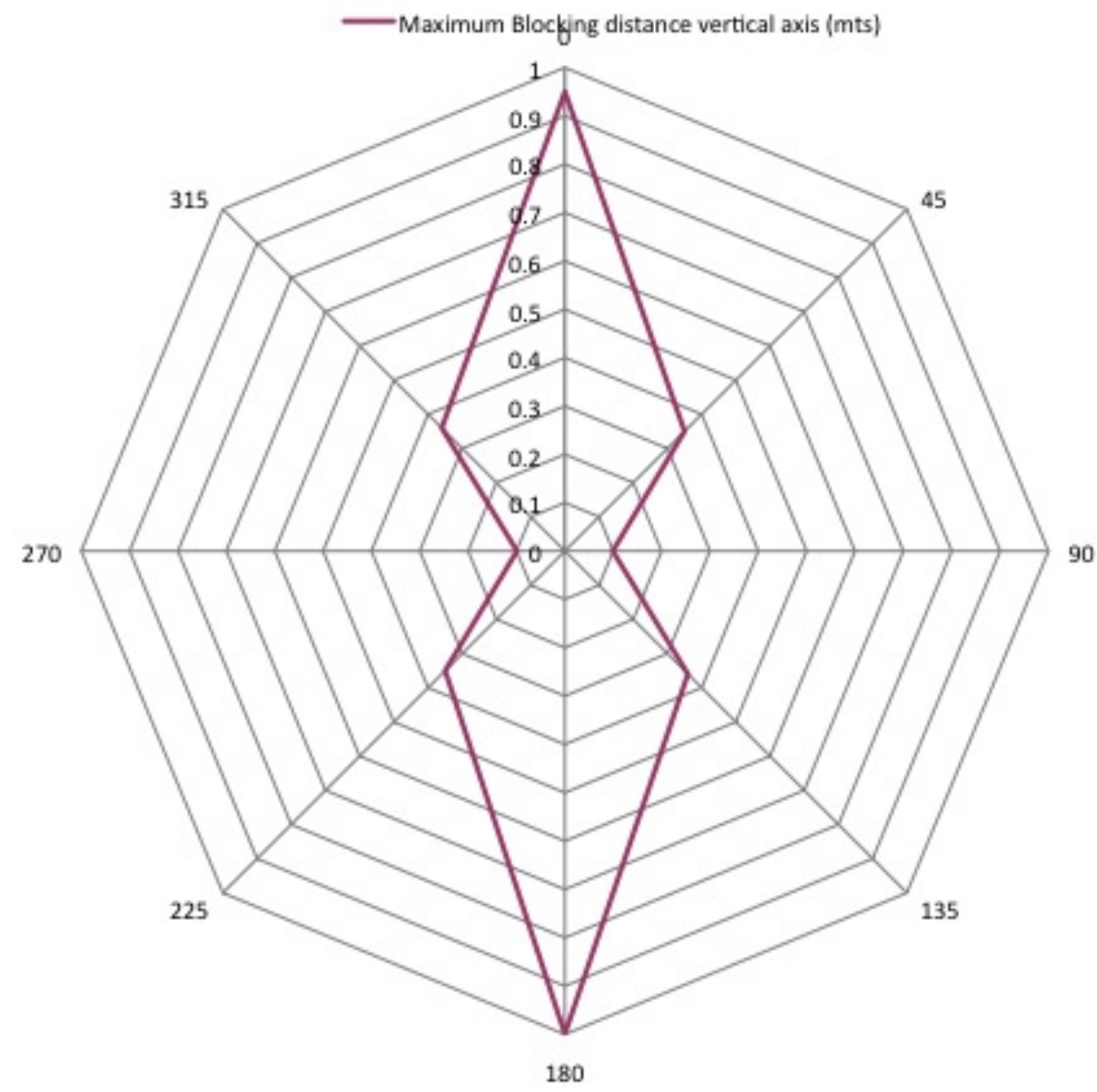

Figure 7.16. Blocking envelope in vertical axis results. 


\section{CHAPTER 8}

\section{BLOCKING READER IN PRACTICE}

\subsection{Introduction}

Blocking Reader can be used to preserve privacy. The ability of the blocking reader to actually block the communication between the Reader and Tag can serve as a device that can be used in places where the privacy of the product should be preserved. In places like warehouse, hospital, residential areas etc the privacy of the products are important. So if there is a blocking reader then the products can be preserved from being read by some third party or unauthorized Readers.

\subsection{Warehouse}

Companies store there products to be marketed in the warehouse. If the products are tagged with RFID tags it would be easy for any person with powerful Reader to read all the Tags and know lot of this information about the demand and sales of the company itself. Now it would become important for the company to preserve the privacy. If there is a low cost Blocking Reader in the warehouse it can prevent any external third party venders to read the products in the warehouse. Hence we can preserve the privacy of the company by preventing the third party from reading the company tags.

Advantages of using the Blocking Reader in the Warehouse

- A low cost product can ensure the privacy.

- Creating a shield around the warehouse.

- Easily configurable parameters like range. 
- Can be controlled from ethernet.

- Isolated envelopes of blocking.

Disadvantages of using the Blocking Reader in the warehouse

- Require multiple Blocking Readers for bigger warehouse.

- Will cause interference to other signals.

- Needs to be turned off even an authorized person needs to read.

\subsection{Blocking Reader Around Human Body}

In this section we will discuss about how Blocking Reader works around Human Body. Previous research has shown that the presence of Human causes deformation in the read zones of a typical passive RFID systems [7]. Human body tissue is a lot of liquids and the major part is water. The property of water is absorption of $\mathrm{RF}$ radiations. Due to the reflection and absorption of these $\mathrm{RF}$ radiation the $\mathrm{RF}$ radiation pattern is too deformed. In the Figure 8.1 shows an simulation of a monopole antenna in presence of Human Body, 0.3m in front of it.

A Blocking Reader can be used to prevent an unauthorized read of a persons RFID enabled credit cards. Also Prevent unauthorized reads of personal things at a public place. An important application will be to block the unauthorized read of your human implanted tags in the public place causing breach of privacy, leading to tracking. 

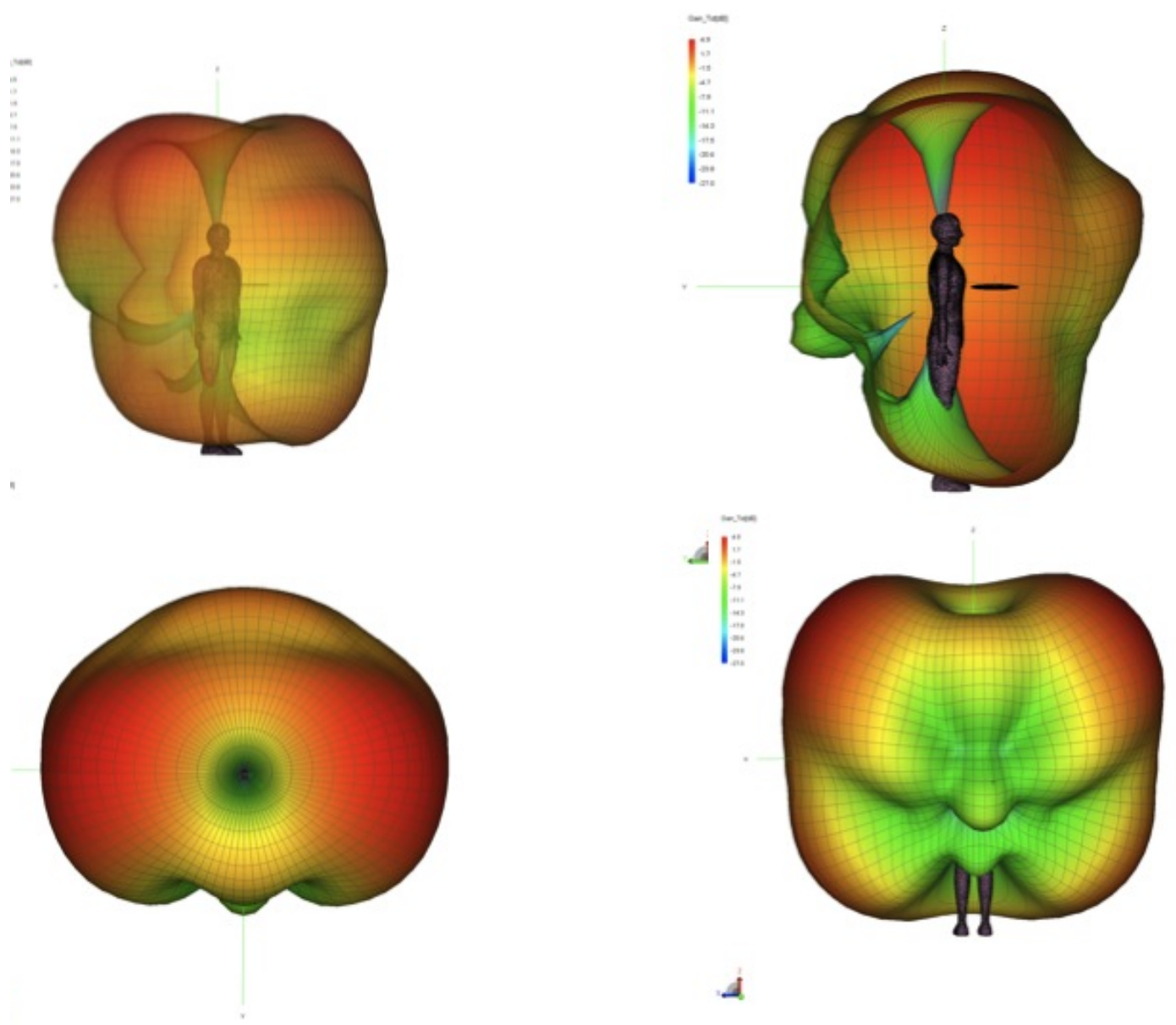

Figure 8.1. FEKO simulation of monopole antenna in front of human body. 


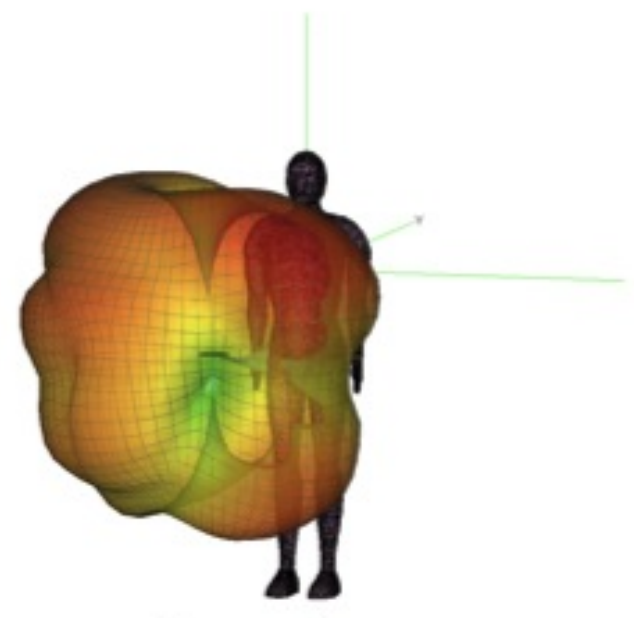

Transparent

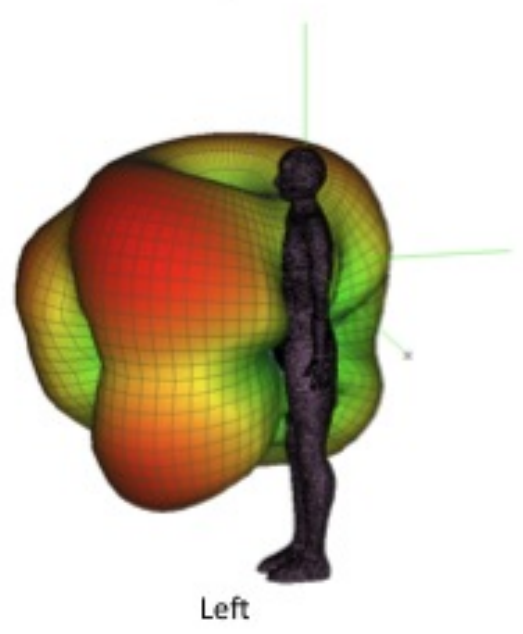

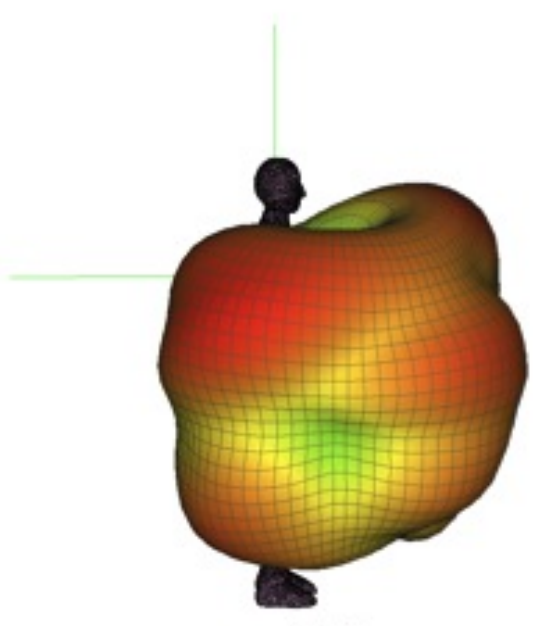

Right

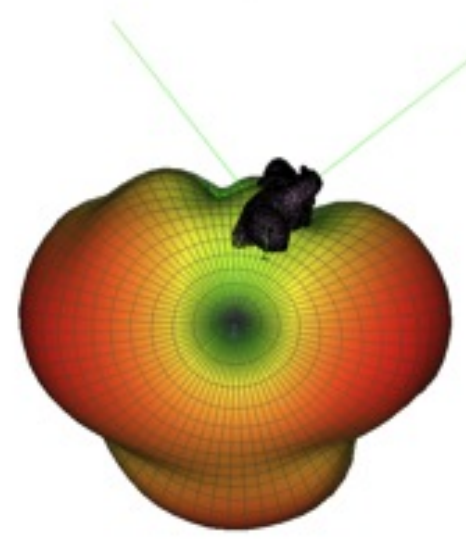

Top

Figure 8.2. FEKO simulation of monopole antenna near hip of human body. 


\subsubsection{Blocking Reader around human body setup}

The experimental setup to verify the Blocking Reader working on a person is done on FEKO as shown in the Figure 8.3. But to verify these we used a 5 gallon filled water bottle for practical experiments instead of a live subject. It is assumed that the 5 gallon water bottle has same effects on the RF radiation patterns. Assumptions are made considering human body is full of fluids. In this setup the Blocking Reader is placed or strapped to a water bottle. The water bottle with the Blocking Reader is rotated around and the Tag is moved from the Blocking Reader towards the Sirit Reader. The maximum blocking distance D is noted.

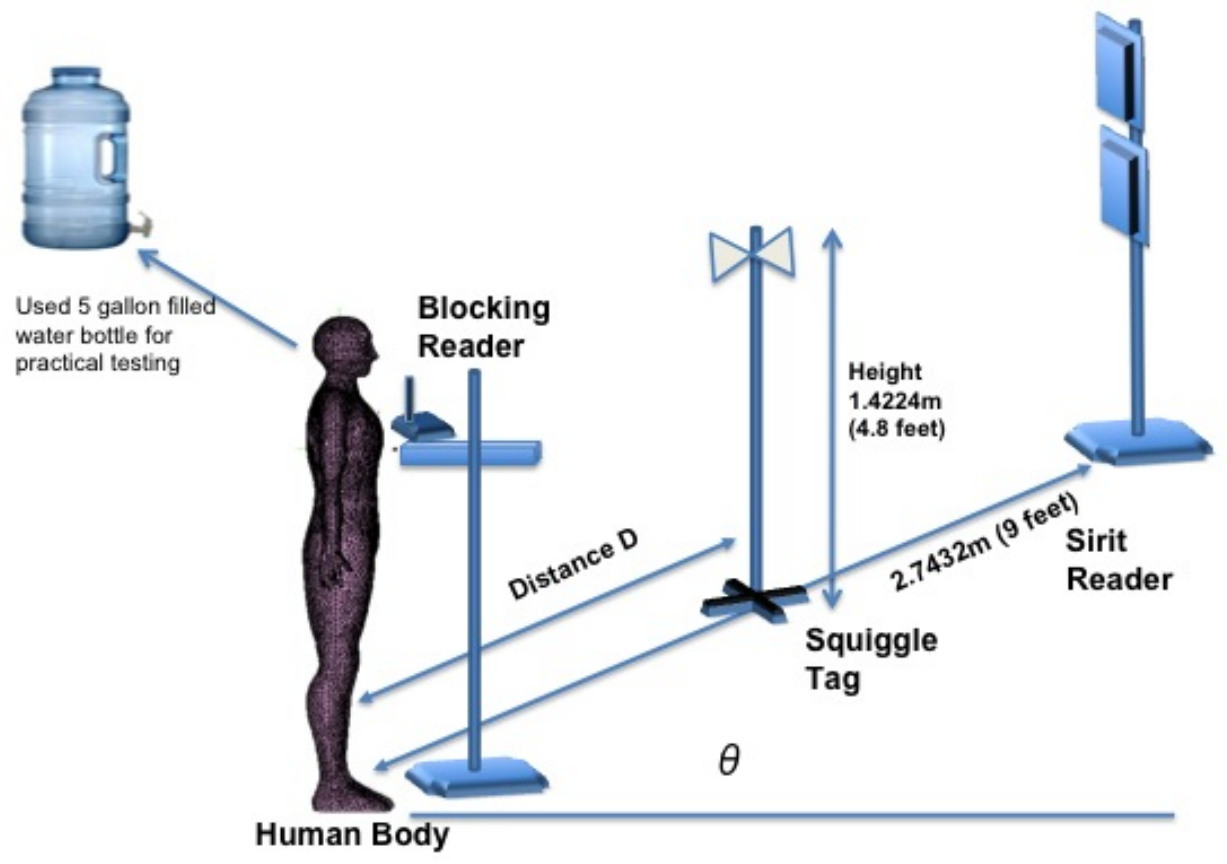

Figure 8.3. Setup for blocking reader around a human body(water bottle) . 


\subsubsection{Results}

The Graph 8.4 shows the maximum distance of blocking around the water bottle. In this we can clearly see a depreciation of blocking range. This is mainly due to the deformation in the radiation patterns caused by absorption and reflection [7]. At 180 degrees the maximum blocking distance is 0 , in this position water bottle is in between the Blocking Reader and Tag. So there is absolutely no communication between Tag and Blocking Reader. So concluding, Blocking Reader can work around person. Human presence had a know limited effect, but results shows considerable blocking envelope around the person.

\section{Maximum Blocking distance $(\mathrm{m})$ around of water bottle}

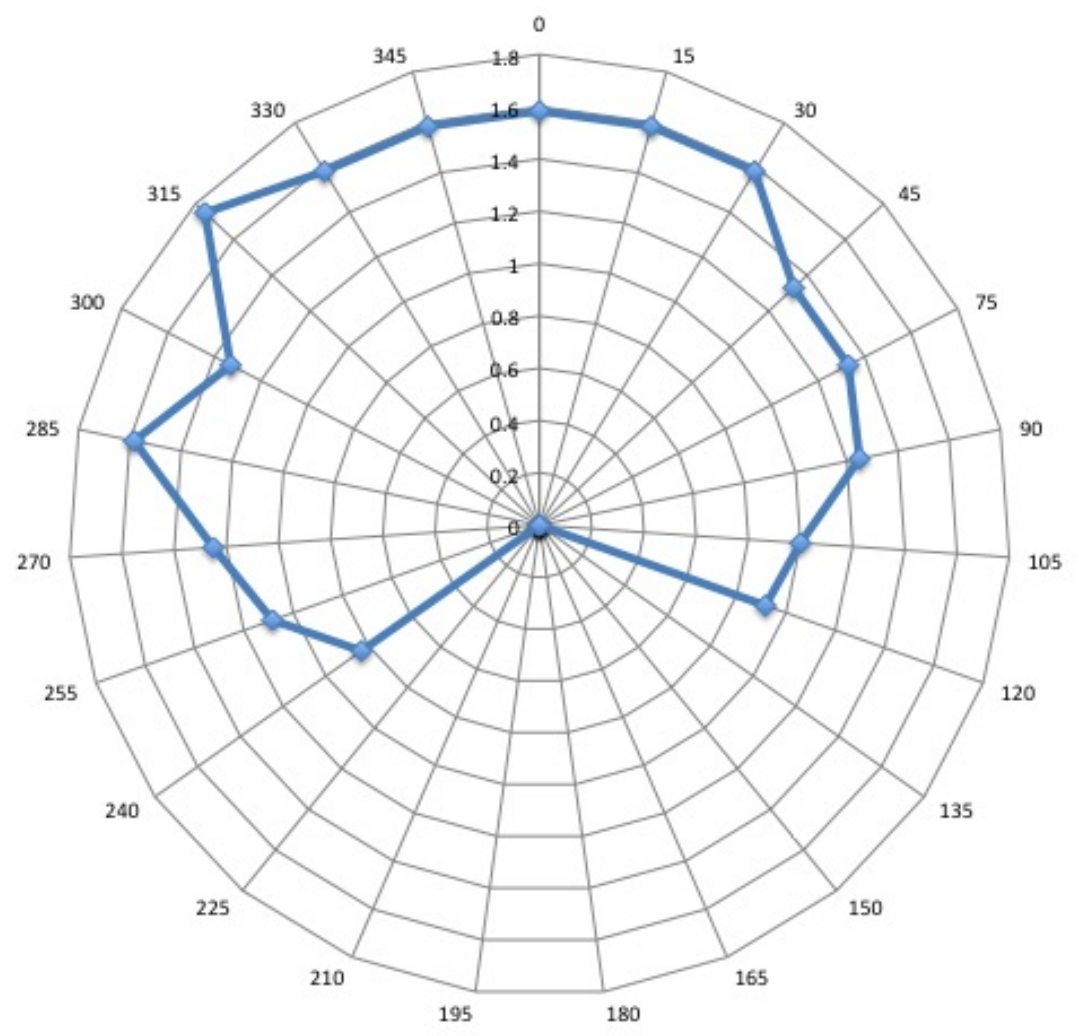

Figure 8.4. Maximum blocking distance measured around water bottle . 


\section{CHAPTER 9}

\section{CONCLUSIONS AND FUTURE WORK}

\subsection{Conclusions}

Blocking Reader creates an envelope around which the Tag is preserved from being read by any unauthorized Readers around. The Blocking Reader can be used to ensure privacy of a passive UHF RFID systems. It uses same protocol as the Readers hence are designed to be FCC compliant. The concept of the Blocking Reader is by capturing the Tag and not allowing other Readers to communicate. This is done by sending series of Query commands. Tags reply to this command with a RN16 whenever it see this command. Hence the communication is always incomplete. When other unauthorized Readers try to communicate with the Tag it just receives a seriesof RN16 values and the TID of the Tag is still secure. Hence blocking the communication.

The Blocking Reader is designed to all the constraints of FCC Part15. The Tags reply to the Blocking Reader with the RN16 value. It follows the same power pattern as the Reader. It matches with the FEKO power patterns for monopole antenna. Blocks the Tag even if the Reader and BR power levels are same. Hence from all these reason we can prove that the Blocking Reader is not jamming but blocking.

The BR creates an envelope around and is comparable to the power radiated. For a power radiated by a Blocking Reader of $19-21 \mathrm{dBm}$ there is a maximum blocking distance of $1.3 \mathrm{~m}$ and for $6-9 \mathrm{dBm}$ power the maximum blocking distance is observed to be $1 \mathrm{~m}$. With all the experiments done is a laboratory environment. A optimum maximum distance is calculated. So It can be concluded that a Blocking reader can 
provide with a good authorized blocking. And can the Blocking envelope can be controllable.

Blocking Reader can be strapped to a person and provide personal privacy for all his Tags. From laboratory experiments I have concluded that it can create an secure envelope of about $1 \mathrm{~m}$ around the person called blocking envelope. This envelope successively blocks any unauthorized Readers trying to read the persons Tags.

I conclude that the Blocking Reader is a Low cost device. With cheap hardware privacy of the RFID systems can be preserved. Also in doing this research we have noticed a potential for using this hardware design for making a full blown Ultra-Low Cost Readers. Hence reducing the cost of a Reader by a factor of 10 .

The concept used here is foolproof. It can easily be used by any RFID systems as it uses the same protocol used by the respective systems. All RFID systems uses inventory round for accessing the TID of the Tag. Hence implementing this concept in inventory round will ensure privacy of other systems also.

\subsection{Future Work}

The Blocking Reader concept can be used for other RFID systems where the protocol has a inventory round. The Blocking Reader Architecture can be used for designing a Ultra-Low Cost UHf RFID Readers. The concept can be implemented on the Tags to make a Tag emulator. A tag emulator emulates the Tags. So if unauthorized Readers trying to access the Tags, Tag emulator will respond instead of the Tag, hence blocking. Like blocker tag we can call it passive blocker reader. If we use this concept on Tag creating a Blocking Reader Tag we can further reduce the cost to few cents. Here a strong wide band Tag is implemented with Blocking Reader protocol. When an unauthorized Reader tries to communicate with your Tag, you will 
be having this Blocking Reader Tag which sends Query when energized. This Query is misunderstood by other Tags in the field for Reader command and continuously send RN 16. Here a Tag is captured by a Tag. So there is a lot of potential for the Blocking Reader. The RFID is a stupendous world yet to be explored. 


\section{REFERENCES}

[1] CC1101: http://focus.ti.com/lit/ds/symlink/cc1101.pdf.

[2] www.rfmd.com/DataBooks/db97/5110.pdf.

[3] Epc global radio frequency identity air interface protocol class 1 generation 2 uhf rfid version 1.0.5, 2004 .

[4] US Patent 6021315. System and method for testing of wireless communication devices.

[5] US Patent 6759863. Wireless radio frequency design and method for testing of integrated circuits and wafer.

[6] Ronald L Rivest Ari Juels and Michael Szydlo. The blocker tag: Selective blocking of rfid tags for consumer privacy. pages 103-111, 2003.

[7] Darmindra D Arumugam, Ananyaa Gautham, Gaurov Narayanaswamy, Nikhil Ayer, and Daniel W Engels. Impact of human presence on the read zones of passive uhf rfid systems. Int. J. Radio Frequency Identification Technology and Applications, 2(1/2):46-63, 2009.

[8] Dramindra D. Arumugam, Ananyaa Gautham, Gaurov Narayanaswamy, and Daniel W. Engels. Impact of RF Radiation on the Human Body in a Passive Wireless Healthcare Environment. IEEE Conference on Ambient Pervasive Healthcare APiPH, 2008.

[9] Rajeev Bansal. Handbook of Engineering Electromagnetics. Marcel Dekker,Inc., 2004. 
[10] Frank Berkers, Jan Goossenaerts, Dieter Hammer, and Hans Wortmann. Human models and data in the ubiquitous information infrastructure. pages 91-104. 2002.

[11] Manish Bhuptani and Shahrammoradpour. RFID Field Guide: Deploying Radio Frequency Identification Systems. Sun Microsystems Press, 2005.

[12] Erik-Oliver Blass, Anil Kurmus, Refik Molva, and Thorsten Strufe. Psp: Private and secure payment with rfid. Cryptology ePrint Archive, Report 2009/181, Apr 2009.

[13] Mike Burmester, Breno de Medeiros, and Rossana Motta. Robust, anonymous rfid authentication with constant key-lookup. In ASIACCS '08: Proceedings of the 2008 ACM symposium on Information, computer and communications security, pages 283-291. ACM, 2008.

[14] M. E. Cates. Yielding and jamming of dense suspensions. pages 3-21. 2006.

[15] David L. Dill, Andreas J. Drexler andAlan J. Hu, and C. Han Yan. Protocol verication as a hardware design aid. pages $522-525$.

[16] Daniel M. Dobkin. The RF in RFID: Passive UHF RFID in Practice. Newnes, pap/cdr edition, September 2007.

[17] Desmond Doran, Alex Hill, Ki-Soon Hwang, and Gregoire Jacob. Supply chain modularisation: Cases from the french automobile industry. In Special section on contextualisation of supply chain networks, 9th International Symposium in Logistics, volume 106, pages 2-11. March 2007.

[18] D. Engels. Review of rfid technology. Texas RF Innovation and Technology Center, 2007.

[19] P. Foster and R. Burberry. Antenna problems in rfid systems. pages 3/1-3/5, 1999. 
[20] K. Fotopoulou and B. W. Flynn. Optimum antenna coil structure for inductive powering of passive rfid tags. In RFID, 200\%. IEEE International Conference on, pages 71-77, 2007.

[21] Minoru Fujimoto. Physics of Classical Electromagnetism. Springer, 2007.

[22] R. Glidden. Design of ultra-low-cost uhf rfid tags for supply chain applications. IEEE Communications Magazine, 42:140-151, 2004.

[23] Lal Chand Godara. Handbook of Antennas In Wireless Communications. CRC Press LLC, 2002.

[24] Ari Juels. The outer limits of rfid security. page 231. 2006.

[25] Ari Juels. Rfid security and privacy: A research survey. IEEE Journal on Selected Areas in Communication, 24(2), February 2006.

[26] Ari Juels and R. Pappu. Squealing Euros: Privacy protection in RFID-enabled banknotes. Springer-Verlag., financial cryptography '03 edition, 2003.

[27] Nemai C. Karmakar, Sushim M. Roy, and Muhammad S. Ikram. Development of smart antenna for rfid reader. In RFID, 2008 IEEE International Conference on, pages 65-73, 2008.

[28] J. S. Kim, K. H. Shin, S. M. Park, W. K. Choi, and N. S. Seong. Polarization and space diversity antenna using inverted-f antennas for rfid reader applications. Antennas and Wireless Propagation Letters, IEEE, 5(1):265-268, 2006.

[29] Heiko Knospe and Hartmut Pohl. Rfid security. Information Security Technical Report, 9(4):39-50, December 2004.

[30] Tae B. Lee, Yeon C. Hong, and Yong H. Kim. Design of active rfid reader for fast recognition time. In 2006 IEEE International Symposium on Industrial Electronics, volume 4, pages 2914-2917, 2006.

[31] Vance Lockton and Richard Rosenberg. Rfid: The next serious threat to privacy. Ethics and Information Technology, 7(4):221-231, December 2005. 
[32] Vance Lockton and Richard Rosenberg. Rfid: The next serious threat to privacy. Ethics and Information Technology, 7(4):221-231, December 2005.

[33] Elgar Fleisch Marin Strassner. The promise of auto-id in the automotive industry. Technical report, Auto-ID Labs, May 2003.

[34] L. Mats, J. T. Cain, and M. H. Mickle. Analysis and synthesis of rfid equivalent circuits through backscatter and ars. In RFID, 200\%. IEEE International Conference on, pages 49-56, 2007.

[35] Ultan Mc Carthy, Gashaw Ayalew, Francis Butler, Kevin Mcdonnell, and Shane Ward. Impact of reader antenna polarisation, distance, inlay design, conveyor speed, tag location and orientation on the coupling of uhf rfid as applied to modified atmosphere packaged meat. Computers and Electronics in Agriculture, August 2009.

[36] Luke Mirowski, Jacqueline Hartnett, and Raymond Williams. An rfid attacker behavior taxonomy. IEEE Pervasive Computing, 8(4):79-84, 2009.

[37] P. Nikitin and K.V Rao. Theory and measurement of backscattering from rfid tags. IEEE-Antennas and Propagation Magazine, 48(6):212-218, 2006.

[38] M. Ohkubo, K. Suzuki, and S. Kinoshita. Rfid privacy issues and technical challenges. ACM Commununication Journal, 48(9):66-71, 2005.

[39] Radu I. Paise and Serge Vaudenay. Mutual authentication in rfid: security and privacy. In ASIACCS '08: Proceedings of the 2008 ACM symposium on Information, computer and communications security, pages 292-299. ACM, 2008.

[40] S. Pete. Passive rfid basics.

[41] S. J. Plimpton and W. E. Lawton. A very accurate test of coulomb's law of force between charges. 50, 1936. 
[42] Xianming Qing and Zhi N. Chen. Proximity effects of metallic environments on high frequency rfid reader antenna: Study and applications. Antennas and Propagation, IEEE Transactions on, 55(11):3105-3111, 2007.

[43] Xianming Qing and Zhi N. Chen. Proximity effects of metallic environments on high frequency rfid reader antenna: Study and applications. Antennas and Propagation, IEEE Transactions on, 55(11):3105-3111, 2007.

[44] E. Rechtin and M. W. Maier. The Art of Systems Architecting. CRC Press, New York, 1997.

[45] R. Rivest S.A. Weis, S. Sarma and D. Engels. Security and privacy aspects of low-cost radio frequency identification systems. 2003.

[46] Daniel W. Engels Sanjay E. Sharma, Stephen A. Weis. Rfid systems. security and privacy implications. Technical report, MIT Auto-ID Center, February 2002.

[47] T. Scharfeld. An analysis of fundamental constraint on low cost passive rfid system design. Master's thesis, MIT, 2001.

[48] L. Sydanheimol, J. Nummetla, L. Ukkonen, J. McVay, A. Hoorfar, and M. Kivikoskil. Characterization of passive UHF RFID tag performance. IEEEAntennas and Propagation Magazine, 50(3):207-212, 2008.

[49] S.A. Weis. Radio frequency identification security and privacy. Master's thesis, MIT, May 2003.

[50] Stephen A. Weis, Sanjay E. Sarma, Ronald L. Rivest, and Daniel W. Engels. Security and Privacy Aspects of Low-Cost Radio Frequency Identification Systems, volume 2802 of Lecture Notes in Computer Science. 2004.

[51] Markus Whn. Electromagnetic Field Theory: a problem solving approach. John Wiley and Sons, 1999.

[52] W.N.Cottingham and D.A.Greenwood. Electricity and Magnetism. Cambridge University Press, 1991. 
[53] N. C. Wu, M. A. Nystrom, T. R. Lin, and H. C. Yu. Challenges to global rfid adoption. Technovation, 26(12):1317-1323, December 2006.

[54] Chuanzhen Zang, Yushun Fan, and Renjing Liu. Architecture, implementation and application of complex event processing in enterprise information systems based on rfid. Information Systems Frontiers, 10(5):543-553, November 2008. 


\section{BIOGRAPHICAL STATEMENT}

Gaurov Narayanaswamy was born in Bangalore, India, in 1983. Currently a researcher in Texas Radio Frequency Innovation and Technology Center at University of Texas at Arlington. Gaurov completed his Bachelors degree in Electronics and Communications at Bangalore in 2006 with honors. Later joined UTA for Masters in Electrical Engineering. Gaurov is voted as Mr. Engineer 2009 for University of Texas Arlington. He is currently a Graduate Teaching Assistant for undergraduate labs. His current research is in the area of Radio Frequency (RF) circuit design, Radio Frequency IDentification(RFID) system design, antenna design, wireless sensor systems, analog/ mixed signal ASIC design. His vision for the technology is to wireless/cordless world where wireless devices are ultra-low cost. 